\title{
Recent progress in fast-ion diagnostics for magnetically confined plasmas
}

\author{
D. Moseev ${ }^{1}$ (D) $\cdot$ M. Salewski ${ }^{2} \cdot$ M. Garcia-Muñoz ${ }^{3} \cdot$ B. Geiger ${ }^{1} \cdot$ M. Nocente No $^{4,5}$
}

Received: 11 August 2017 / Accepted: 2 August 2018 / Published online: 17 September 2018

(c) The Author(s) 2018

\begin{abstract}
On the road to a fusion reactor, a thorough control of the fast-ion distribution plays a crucial role. Fusion-born $\alpha$-particles are, indeed, a necessary ingredient of selfsustained burning plasmas. Recent developments in the diagnostic of fast-ion distributions have significantly improved our predictive capabilities towards future devices. Here, we review key diagnostic techniques for confined and lost fast ions in tokamak and stellarator plasmas. We discuss neutron and gamma-ray spectroscopy, fast-ion D- $\alpha$ spectroscopy, collective Thomson scattering, neutral particle analyzers, and fast-ion loss detectors. The review covers physical principles of each diagnostic, sensitivities, basic setups, and operational parameters. The review is largely (but not exclusively) based on the contributions from ASDEX Upgrade and JET. Finally, we discuss integrated data analysis of fast-ion diagnostics by velocity-space tomography which allows measurements of $2 \mathrm{D}$ velocity distribution functions of confined fast ions.
\end{abstract}

Keywords Diagnostics $\cdot$ Fast ions $\cdot$ Fusion plasmas

D. Moseev

dmitry.moseev@ipp.mpg.de

M. Salewski

msal@fysik.dtu.dk

M. Garcia-Muñoz

mgm@us.es

B. Geiger

benedikt.geiger@ipp.mpg.de

M. Nocente

massimo.nocente@mib.infn.it

1 Max-Planck-Institut fuer Plasmaphysik, Greifswald, Germany

2 Technical University of Denmark, Kongens Lyngby, Denmark

3 University of Seville, Seville, Spain

4 Dipartimento di Fisica “G. Occhialini”, Universita di Milano-Bicocca, Milano, Italy

5 Istituto di Fisica del Plasma "P. Caldirola", Consiglio Nazionale delle Ricerche, Milano, Italy 


\section{Introduction}

Fast ions are a crucial ingredient of a burning fusion plasma as they constitute an essential source of energy to heat the plasma and thus to sustain the fusion burn. They are also significant sources of momentum and current in tokamak plasmas. In present fusion devices, the main fast-ion sources are Neutral Beam Injectors (NBI) and electromagnetic wave heating systems in the Ion Cyclotron Range of Frequencies (ICRF). In a fusion plasma, the main fast-ion source will be the fusion reaction $\mathrm{D}(\mathrm{T}, \mathrm{n}) \alpha$ which generates $\alpha$-particles at $3.5 \mathrm{MeV}$.

However, there are several transport mechanisms that can lead to a fast-ion redistribution and eventually loss before the fast ions have slowed down to the bulk plasma energies through Coulomb collisions (Heidbrink and Sadler 1994; Fasoli et al. 2007). A fast-ion redistribution is typically accompanied by a degradation of the fast-ion heating and current drive efficiency and thus of the fusion reactor performance. If sufficiently intense and localized, a fast-ion loss may even damage the integrity of the first wall of the device (Duong et al. 1993). Magnetohydrodynamic (MHD) fluctuations are the main cause of fast-ion transport (García-Muñoz et al. 2009a). Among others, Alfvén Eigenmodes (AEs) Wong et al. (1991); Heidbrink et al. (1991); Kimura et al. (1998); Berk et al. (2001); Sharapov et al. (2001); Shinohara et al. (2004); Snipes et al. (2005); Van Zeeland et al. (2006); García-Muñoz et al. (2011), Neoclassical Tearing Modes (NTMs) (Zweben et al. 1999; Carolipio 2002b; García-Muñoz et al. 2007), sawtooth crashes (Kolesnichenko and Yakovenko 1996; Van Zeeland et al. 2010; Salewski et al. 2016b), and Edge Localized Modes (ELMs) (García-Muñoz et al. 2013a, b) can reduce the fast-ion density by up to $50 \%$ of the classically expected density. The wave-particle interaction causing this fast-ion transport depends on the nature of the fluctuations and fast-ion orbital characteristics. To understand the physics mechanisms underlying the observed MHDinduced fast-ion transport, accurate time-resolved measurements of the fast-ion distribution in phase space are needed. Such measurements are essential to validate and challenge present theories, which advances our understanding and predictive capabilities towards future fusion devices. The harsh environment in a fusion device complicates this task. Recent breakthroughs in the diagnostic of confined and lost fast ions have allowed measurements of MHD-induced fast-ion transport with an unprecedented level of detail and accuracy.

In this review, recent developments of key fast-ion diagnostic techniques in fusion plasmas are presented together with a brief discussion of their prospects for future devices. The physics of energetic ions has been discussed in several review papers (Gorelenkov et al. 2014; Heidbrink and Sadler 1994; ITER Physics Expert Group 1999; Fasoli et al. 2007; Pinches et al. 2015; Sharapov et al. 2013), and, after decades of research, is still incomplete. Here, we do not discuss the physics of fast ions, but focus on their diagnostic, which has proven to be a challenging topic by itself. We explain physical principles, sensitivity, and limitations of the diagnostics, as well as engineering solutions which are used for obtaining optimal measurements. The review covers neutron and gamma-ray spectroscopy, fast-ion $\mathrm{D}_{\alpha}$ spectroscopy, collective Thomson scattering, neutral particle analyzers, and fast-ion loss detectors. 
There are comprehensive reviews on diagnostics in magnetized high-temperature plasma in general (Gentle et al. 1995) and on the diagnostics applicable to ITER in particular (ITER Physics Expert Group 1999; Donné 2007). We complement the content of these papers by looking in specifically fast-ion diagnostics in greater detail. There are also reviews on particular fast-ion diagnostics: fast-ion $\mathrm{D}_{\alpha}$ spectroscopy (Heidbrink 2010) and neutral particle analyzer (Medley et al. 2008). We focus on recent developments of those diagnostics and relate them in the framework of integrated data analysis by velocity-space tomography.

It is of interest to measure fast ions that are confined in the plasma by the magnetic field as well as fast ions that are lost from the plasma. The most common diagnostics for confined fast ions are neutron emission spectroscopy (NES) or the simpler neutron counters, $\gamma$-ray spectroscopy (GRS), fast-ion $\mathrm{D}_{\alpha}$ spectroscopy (FIDA), neutral particle analyzers (NPA), and collective Thomson scattering (CTS). Fast-ion loss detectors (FILD) measure fast ions on orbits leaving the plasma either due to the birth on unconfined orbits or due to the interaction with the plasma and waves therein. The different fast-ion diagnostics observe various parts of phase space with different sensitivities and thus provide complementary information about the fastion phase-space distribution function.

We may divide the confined ion diagnostics into two main groups: active and passive diagnostics. The active measurements require the injection of a beam, either of radiation or of particles, and the measured signal depends on parameters of the plasma and of the injected beam. The beam can be perturbative or non-perturbative. For example, the NBI required for FIDA and for active NPA is often a part of the heating scenario, so that these diagnostics are practically non-perturbative. If the beam is not used for heating, short pulses of the probe NBI need to be injected. The probe beam for CTS consists of unabsorbed mm-wave radiation with a frequency between or below the electron cyclotron emission (ECE) harmonics. Hence, CTS does not perturb the plasma.

In passive measurements, one monitors radiation or particles naturally emitted by the plasma, and hence, they never perturb the plasma. The passive fast-ion diagnostics that we treat in this review are NES, neutron counters, GRS, passive FIDA, and passive NPA. In GRS one measures the energy spectrum of $\gamma$-rays originating from fusion reactions. In NES, one detects neutrons originating from fusion reactions and measures, depending on the type of the detector, a variety of quantities that can be related to the neutron energies. Passive FIDA diagnoses the Balmer-alpha emission originating from the charge-exchange reactions between the background neutrals at the edge and fast ions. In passive NPAs, one measures the energy spectrum of escaping neutral particles that have been generated in this charge-exchange reaction.

The division into active and passive diagnostics is reflected in the achievable spatial resolution of the measurements. The probe beam of the active measurements and the line-of-sight of the detector are arranged to intersect at the desired measurement location in the plasma. The spatial resolution of the active measurements is, therefore, largely determined by the sizes of the probe beam and the line-of-sight and by their intersection angle. For FIDA and NPA, the lines-of-sight can be chosen to be narrow, whereas the size of the heating beam is determined by the desired heating performance rather than the diagnostic 
needs. Microwave-based CTS usually uses the available infrastructure of existing Electron Cyclotron Resonance Heating (ECRH) systems. The sizes of the beams depend on the propagation of the microwaves through the plasma and the geometry of the diagnostic setup.

The passive diagnostics NES, GRS, and passive FIDA and NPAs always measure along their entire line-of-sight. Nevertheless, the measurement can be strongly dominated by only parts of the line-of-sight. The $\gamma$-ray and neutron emission is strongest in the plasma center and weakens substantially towards the plasma edge. In contrast to that, passive NPAs have a strong contribution from the plasma edge, where there are high densities of donor neutrals. This spatial weighting can act as practical spatial resolution. $\gamma$-rays and neutrons at JET are monitored along several lines-of-sight, such that their 2D emission profiles in the poloidal plane can be found by tomographic inversion.

Fast-ion loss detectors (FILDs) are charged particle collectors located in the direct proximity of the plasma edge. They measure fluxes of charged particles. In contrast to the diagnostics of confined fast ions, the FILD diagnostic measures a distribution of energies and pitches of the lost ions on the scintillator plate. The pitch is defined as

$$
p=\frac{\mathbf{v} \cdot \mathbf{B}}{|\mathbf{v}||\mathbf{B}|}
$$

where $\mathbf{v}$ is the particle velocity and $\mathbf{B}$ is the magnetic field. However, if the plasma current and the toroidal magnetic field point in opposite directions, the sign of the pitch is sometimes reversed by convention. Often, we also refer to the pitch angle $\arccos p$. The original orbit of the detected particles can be calculated in orbit-following simulations. The diagnostic is compact and is often installed on a manipulator. By moving the detector on the manipulator, different spatial positions can be probed.

The velocity-space sensitivities of the confined fast-ion diagnostics have only very recently been understood and quantified. The velocity-space observation regions depend on the diagnostic principle and the gyro-motion of the energetic particles. Often, one can draw conclusions on the velocity component along the line-of-sight of the diagnostic from the detected signal. High-energy detections (frequency upshift for radiation) indicate motion of the energetic particle towards the detector, whereas low-energy detections indicate motion away from the detector. For two-step reactions emitting $\gamma$-rays, this is not strictly true, but there is still a bias in this direction.

This paper is organized as follows: Sect. 2 describes the NES and GRS diagnostics; the diagnostics based on charge exchange, NPA and FIDA, are explained in Sect. 3; and Sect. 4 is devoted to CTS. The diagnostic for lost ions, FILD, is described in Sect. 5. Section 6 discusses velocity-space tomography as tool for integrated data analysis of the available measurements. Section 7 concludes this paper. 


\section{Neutron and gamma-ray emission spectrometry}

In this section, we review diagnostics based on the measurements of various nuclear reaction products formed in high-temperature plasmas. Up to now, this is the most mature family of diagnostics. Fusion product diagnostics have recently been reviewed (Sasao et al. 2008), and we here update and expand on this work. This review is focused primarily on the spectroscopy applications of this group of diagnostics, as this is the most direct method to gain access to the underlying distribution function of the energetic ions. The use of non-spectroscopic neutron detectors for fast-ion studies is also briefly discussed. Basic neutron measurements are exploited in virtually all major fusion devices, but the spectroscopic application of neutron and $\gamma$-ray emission for fastion studies has been mostly performed at the JET tokamak.

\subsection{Physics principles}

Neutron measurements Neutron emission arises from fusion reactions of the plasma constituents, most notably the $\mathrm{d}(\mathrm{d}, \mathrm{n})^{3} \mathrm{He}$ and $\mathrm{t}(\mathrm{d}, \mathrm{n})^{4} \mathrm{He}$ reactions in deuterium (D) and deuterium-tritium (DT) plasmas, respectively. Neutron measurements were originally intended as a way to determine the fusion power yield as well as its profile. Measurements can be coarsely divided into two groups depending on their aim to measure the the number of the fusion neutrons or a spectrum that is related to their energies. Calibrated flux detectors were originally intended to determine the neutron yield produced in a plasma discharge, which is in turn proportional to the fusion power (Jarvis 1994). Neutron spectrometers were considered for measurements of the bulk-ion plasma temperature $T_{i}$, based on the theoretical derivation (Lehner and Pohl 1967; Faust and Harris 1960; Brysk 1973) that the width of the spectrum is proportional to $\sqrt{T_{\mathrm{i}}}$. Another early application of the spectroscopy technique was aimed at determining the shift of the spectrum, which tells whether fusion reactions are of thermonuclear origin or not (Strachan et al. 1979). The first unambiguous determination of $T_{i}$ from neutron spectroscopy measurements was performed later by Fisher et al. (1983).

The application of neutron measurements to fast-ion physics studies became possible only later with the routine application of systems delivering MW of auxiliary heating power. In this case, besides neutron counters, the development of high-resolution spectrometers specifically tailored to study the fast-ion distribution function was possible. At a fundamental level, fast ions generate a neutron population with energies exceeding those expected from a purely thermal plasma. This follows from the application of energy and momentum conservation to the $d(d, n)^{3} \mathrm{He}$ and $t(d, n)^{4} \mathrm{He}$ fusion reactions and is mathematically expressed by an equation relating the neutron energy $E_{\mathrm{n}}$ to the known motional state of the reactants (Brysk 1973):

$$
E_{\mathrm{n}}=\frac{1}{2} m_{\mathrm{n}} V_{c m}^{2}+\frac{m_{\mathrm{n}}}{m_{\mathrm{n}}+m_{\mathrm{f}}}(Q+K)+V_{\mathrm{cm}} \cos \theta\left(\frac{2 m_{\mathrm{n}} m_{\mathrm{f}}}{m_{\mathrm{n}}+m_{\mathrm{f}}}(Q+K)\right)^{1 / 2}
$$


Here, $m_{\mathrm{n}}$ and $m_{\mathrm{f}}$ indicate the masses of the neutron and the second product of the fusion reaction, respectively. $\mathbf{V}_{\mathbf{c m}}=\left(m_{1} \mathbf{v}_{\mathbf{1}}+m_{2} \mathbf{v}_{\mathbf{2}}\right) /\left(m_{1}+m_{2}\right)$ and $K=1 / 2 \mu v_{\text {rel }}^{2}$ are the center-of-mass $(\mathrm{cm})$ velocity and relative kinetic energy of the two reactant ions, described by their masses $m_{1}$ and $m_{2}$ and velocities $\mathbf{v}_{\mathbf{1}}$ and $\mathbf{v}_{\mathbf{2}} \cdot \mathbf{v}_{\text {rel }}=\mathbf{v}_{\mathbf{2}}-\mathbf{v}_{\mathbf{1}}$ is the relative velocity and $\theta$ indicates the angle between $\mathbf{V}_{\mathbf{c m}}$ and the neutron velocity vector in the $\mathrm{cm}$ frame, which depends on the angle between the line-of-sight of the diagnostic and the magnetic field.

For purely thermal plasmas, the neutron spectrum that is calculated from Eq. 2 is approximately Gaussian with a width proportional to the square root of the ion temperature (Faust and Harris 1960). The center of the spectrum is at 2.5 and $14 \mathrm{MeV}$ for $\mathrm{d}(\mathrm{d}, \mathrm{n})^{3} \mathrm{He}$ and $\mathrm{t}(\mathrm{d}, \mathrm{n})^{4} \mathrm{He}$ neutrons, respectively.

Deformations of the Gaussian shape with the appearance of tails at both ends of the spectrum occur whenever the fuel-ion distribution function has suprathermal components, for example, as a consequence of NBI or ICRF heating. Figure 1 shows a calculation of the neutron spectrum produced by fusion reactions among ions described by the distribution function displayed to the left. We assume that neutrons are observed along an orthogonal line-of-sight with respect to the magnetic field direction. The fuel ions are here described by a Maxwellian distribution with a temperature of $T_{i}=10 \mathrm{keV}$ and a density of $n_{\mathrm{e}}=10^{20} \mathrm{~m}^{-3}$. The additional suprathermal ion population has an assumed tail temperature of $200 \mathrm{keV}$ and a relative density as little as $0.01 \%$, which is here used as a mock-up for the effects of ICRF heating on the fuel-ion distribution function. We also separately consider the corresponding signatures in the neutron spectrum from D and DT plasmas. In both cases, tails appear at both ends of the spectrum. Experimentally, however, it is only the high-energy tail that can be used for diagnostic applications as the low-energy signature is often altered by scattered neutrons (see Sect. 2.2). Fast-ion studies by neutron spectroscopy, therefore, detect the high-energy tail in the spectrum by use of suitable instruments (see Sect. 2.2). The magnitude of the suprathermal ion effects
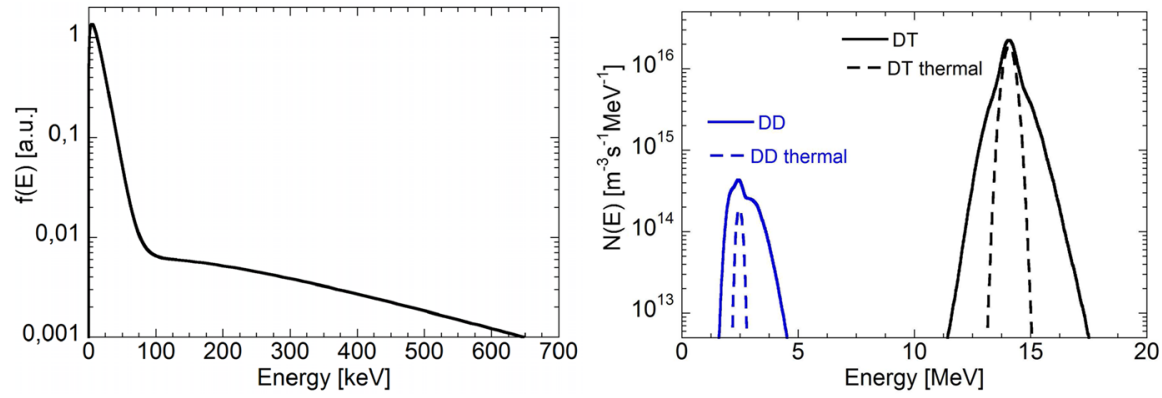

Fig. 1 Left: fuel-ion distribution function with external heating. We assume that the plasma has $T_{\mathrm{i}}=10$ keV bulk-ion temperature and $n_{\mathrm{e}}=10^{20} \mathrm{~m}^{-3}$ density. A $0.01 \%$ fraction of the fuel ions is driven to a tail temperature of $200 \mathrm{keV}$ by the auxiliary heating. Right: expected neutron spectrum produced in D and DT plasmas by reactions among ions described by the distribution function shown to the left as calculated by the GENESIS code (Nocente 2012; Tardocchi et al. 2011) (solid lines). For comparison, the neutron spectrum expected from bulk fuel ions at thermal equilibrium and with $T_{\mathrm{i}}=10 \mathrm{keV}$ is also shown by dashed lines 
in the neutron spectrum is very different for D and DT plasmas. In the former case, a fast-ion population often leads to a significant enhancement of the neutron rate, as the cross section of the $d(d, n)^{3}$ He reaction is a monotonic function of energy up to about $1 \mathrm{MeV}$ in the $\mathrm{cm}$ frame (Nocente et al. 2010a). In the latter case, instead, there is certainly a modification of the shape of the spectrum, but the enhancement of the reactivity is modest, mostly because fast ions with energies exceeding that of the resonance in the cross section (at about $70 \mathrm{keV}$ in the $\mathrm{cm}$ frame) add little to the neutron yield.

Although the dominant fast-ion application of neutron measurements is to determine the effect of the heating systems on the fuels deuterium and tritium, in some special cases, non-fuel energetic ions can also lead to a tail in the neutron spectrum. This occurs, because the fuel-ion distribution function is distorted at high energies (say, more than $100 \mathrm{keV}$ ) when fast non-fuel ions in the MeV-range collide elastically with the fuel ions as they slow down in the plasma (Nocente et al. 2013a). A notable application is the possibility to measure the $\alpha$-particle distribution function by the observation of low-amplitude (approx $10^{-4}$ ), high-energy tails in the neutron spectrum. These are born from the so-called $\alpha$-particle knock-on process, i.e., (mostly) head-on nuclear elastic scattering collisions between $\alpha$-particles in the $\mathrm{MeV}$ range and fuel ions. The $\alpha$-particle knock-on tail has been used to assess classical slowing down of the $\alpha$-particles in DT experiments at JET (Kaellne et al. 2000) by means of a dedicated neutron detector with high dynamic range sensitivity (see Sect. 2.2).

Besides spectrometry, measurements of the neutron yield and profile can also contribute to studies of fast fuel ions. Although the focus of this section is on spectroscopy, for the sake of completeness, we briefly mention these applications as well and we refer to Wolle (1999) for a comprehensive overview. When auxiliary heating systems are used, an enhancement of the neutron yield is expected, particularly in deuterium plasmas. Calculations of the enhancement based on a neoclassical model of the power deposition are often compared with experimental data. In many cases, when the fast-ion content in the plasma is significant, a discrepancy is seen, which may indicate that fast-ion physics effects are at play [see, for example, Fig. 2c, of Carolipio et al. (2002a)]. However, it is often very difficult to exploit neutron-yield measurements alone for a deeper understanding, as the cause for the discrepancy can depend on many interlinked parameters and physics mechanisms, which require additional diagnostic systems to decouple. When fast-ion studies are the aim of the experiment, additional useful information comes from the neutron profile. Typically, neutron profile measurements are used especially to measure modifications of the neutron emission, e.g., in response to sawteeth and fishbones effects on the fast ions (Cecconello et al. 2010; Jarvis 1994).

Finally, a less widespread but useful method concerns the measurements of the so-called triton burn-up neutrons (TBN) (Nishitani et al. 1996; Frenje et al. 1998; Conroy et al. 1988; Heidbrink 1983, 1984). These are $14 \mathrm{MeV}$ neutrons that are born in the $\mathrm{t}(\mathrm{d}, \alpha) \mathrm{n}$ reaction in a deuterium plasma, where tritons with an initial energy of $1 \mathrm{MeV}$ are those naturally produced in the fusion reaction $\mathrm{d}(\mathrm{d}, \mathrm{p}) \mathrm{t}$, which has a rate comparable to $\mathrm{d}(\mathrm{d}, \mathrm{n})^{3} \mathrm{He}$. The abundance of the burn-up depends on plasma parameters, but it is typically of the order of $1 \%$ or less, i.e., a small fraction of the much 
more abundant $2.5 \mathrm{MeV}$ neutrons. Since the TBN emission occurs as the $1 \mathrm{MeV}$ tritons slow down in a bulk deuterium plasma, the time trace (Nishitani et al. 1996; Conroy et al. 1988) of the TBNs is a diagnostic method to assess triton confinement. In tokamaks, early TBN experiments were used to assess the classical confinement of the energetic tritons (Conroy et al. 1988; Nishitani et al. 1996), but they have been progressively replaced by dedicated physics studies that rely on external heating systems as the source of fast ions. The reason is that the fast-ion content is higher in this latter case and there is a greater flexibility in the possibility to tailor the fastion distribution function in different ways. TBN measurements are, however, still of great relevance for stellarator research in deuterium plasmas. In these devices, it is much more challenging to generate ions in the $\mathrm{MeV}$ range by auxiliary heating. TBN measurements can anticipate some physics aspects of the much debated $\alpha$-particle confinement capability of non-axisymmetric toroidal machines. For example, TBNs are expected to play an important role for the physics program of the recently launched deuterium phase of the Large Helical Device (Isobe et al. 2010).

Gamma-ray measurements Gamma-ray measurements are emerging as an essential tool to study fast-ion physics in the MeV range (Kiptily et al. 2002, 2006; Tardocchi et al. 2013). The reactions leading to $\gamma$-ray emission can be divided into two categories, namely, one-step and two-step reactions. In one-step reactions, i.e., those of the type $a(b, \gamma) c$, the two light nuclei $a$ and $b$ merge to form the heavier nucleus $c$. The excess energy that comes from the mass difference $\Delta m=m_{a}+m_{b}-m_{c}$ is released as a $\gamma$-ray with the energy $E_{\gamma}=\Delta m c^{2}$ in the center-of-mass frame. Notable examples are $\mathrm{d}(\mathrm{d}, \gamma)^{4} \mathrm{He}$ and $\mathrm{t}(\mathrm{d}, \gamma)^{5} \mathrm{He}$.

Two-step reactions instead involve a fast ion $f$ and an impurity $i$. In the first stage, the fast ion reacts with the impurity to produce a heavy nucleus $X$ and a light product $c$, i.e., $\mathrm{i}(\mathrm{f}, \mathrm{c}) \mathrm{X}$. Impurities are often found naturally in fusion devices due to erosion of materials in the first wall. The impurities can also be injected on purpose, for example, using pellets or evaporation. In some cases, $\mathrm{X}$ can be born in an excited nuclear state that, on a time scale of some picoseconds or less, de-excites with the emission of $\gamma$-ray radiation (second step). If the $\gamma$-ray is detected, it can be used to infer information on the fast ion that started the two-step process. A notable example is here the ${ }^{9} \mathrm{Be}(\alpha, \mathrm{n}){ }^{12} \mathrm{C}^{*}$ reaction, where ${ }^{12} \mathrm{C}$ born on its first excited state emits a $\gamma$-ray at a characteristic energy of $4.44 \mathrm{MeV}$. In ITER, Be is a first-wall material.

Historically, one-step $\gamma$-ray reactions were the first to be proposed for diagnostic purposes around the $1980 \mathrm{~s}$, but as for neutron measurements, they were initially intended as a means to derive the bulk-ion temperature $T_{i}$ in the plasma core rather than for fast-ion applications (Cecil and Newman 1984). In this case, $T_{i}$ can in principle be derived from the broadening of the $\gamma$-ray peak shape or from its shift with respect to its nominal energy $E_{\gamma}$. In practice, as fusion neutron diagnostics developed, neutrons soon became the reference technique to measure core $T_{i}$, as neutrons are by far more abundant thanks to the comparably higher production cross sections.

One-step $\gamma$-ray reactions are, however, nowadays useful for fast-ion measurements, especially when ICRF waves are injected into the plasma to accelerate hydrogen. In this case, rather than the $\mathrm{d}(\mathrm{d}, \gamma)^{4} \mathrm{He}$ and $\mathrm{t}(\mathrm{d}, \gamma)^{5} \mathrm{He}$ reactions, the $\mathrm{d}(\mathrm{p}, \gamma)^{3} \mathrm{He}$ (Nocente et al. 2012a) and $\mathrm{d}(\mathrm{d}, \gamma)^{4} \mathrm{He}$ (Kiptily 2015) emissions between fast protons and bulk deuterium or tritium are of relevance in D and DT plasmas, 
respectively. As for $T_{i}$ applications, information on the fast-proton distribution function resides in the position and shape of the peak. Careful modelling is required to extract quantitative information from the spectral shape as the simple analytical formulas that apply to Maxwellian plasma [(see, for example, Cecil and Newman (1984)] very often break at the typical energies of the fast ions found in the present tokamaks (Nocente et al. 2015a). An important advantage for the application of onestep reactions to fast-proton studies is an about 100 times higher emission compared to the same reactions among thermal ions, as the cross sections for $\mathrm{d}(\mathrm{p}, \gamma)^{3} \mathrm{He}$ and $\mathrm{d}(\mathrm{d}, \gamma)^{4} \mathrm{He}$ monotonically increase up to the MeV energy range.

Two-step reactions are even more useful for fast-ion applications than one-step reactions for essentially two reasons. First, the cross sections are generally higher by a factor 100 or more. Second, there is a large variety of two-step reactions that can occur in a plasma, which implies that different types of fast ions ( $p,{ }^{3} \mathrm{He}, \alpha$ etc.) can be studied by the two-step $\gamma$-ray emission they produce, even simultaneously. Unlike one-step reactions, which can in principle be used also to extract parameters of the bulk plasma (e.g., core $T_{i}$ ), two-step processes require ions in the $\mathrm{MeV}$ range. The cross sections are essentially negligible below a few hundred $\mathrm{keV}$ and often have energy thresholds (Kiptily et al. 2002).

Information on the fast ions at different levels of detail can be extracted from the measured $\gamma$-ray emission spectrum. At the most basic level, the identification of the mean energy of peaks in the spectrum is used to assess that a specific two-step reaction occurs in the plasma. This in turn establishes that fast ions with energies exceeding that of the reaction threshold are confined in the plasma. For example, the observation of the $4.44 \mathrm{MeV}$ peak from the ${ }^{9} \mathrm{Be}(\alpha, \mathrm{n}){ }^{12} \mathrm{C}^{*}$ reaction indicates that $\alpha$-particles with energies exceeding $1.9 \mathrm{MeV}$ are found in the plasma, as this is the effective threshold above which the cross section becomes substantial for ${ }^{9} \mathrm{Be}(\alpha, \mathrm{n}){ }^{12} \mathrm{C}^{*}$ (Nocente et al. 2012b).

At a more detailed level, as for neutrons, modelling of the emission can be performed to extract quantitative information on fast ions from the measurements. Since the heavy nuclei of two-step reactions can be born in several excited states, more than one peak from the same reaction can be emitted by the different possible transitions between the excited states. Examples are the ${ }^{12} \mathrm{C}\left({ }^{3} \mathrm{He}, \mathrm{p} \gamma\right){ }^{14} \mathrm{~N}$ (Tardocchi et al. 2011) and the ${ }^{12} \mathrm{C}(\mathrm{d}, \mathrm{p} \gamma){ }^{13} \mathrm{C}$ (Nocente et al. 2012b) reactions, which have been used to study ${ }^{3} \mathrm{He}$ and $\mathrm{d}$ ions in the $\mathrm{MeV}$ range, respectively. In this case, the ratio of peak amplitudes from the same reaction depends on the individual cross sections to populate each excited state and its value can change depending on the fastion energy. An important observation is that the ratio is independent of the fast-ion and impurity densities. In practical applications, a model of the fast-ion distribution function is used as input to calculate the expected peak ratio for comparison with measurements, for example, to determine the tail temperature that best describes fast ions in ICRF acceleration experiments. Besides the peak ratio, the measured absolute intensity of the emission can be used to put further constraints on the fastion energy distribution. This, however, requires an independent measurement of the impurity density, which is often not accurately known.

For a reactor, measurements of the ratios of different peaks from the same reaction and at sufficient energy resolution are well within the present capabilities of 
high-resolution detectors (see the section on instrumentation). This, depending on the reaction, allows a first validation of the spectral properties of the fast-ion energy distribution on a relative scale, by comparison between synthetic diagnostics and actual data. The absolute quantification of the fast-ion density would also be desired in a reactor, but this depends on the absolute flux impinging on the detector and on information on the impurity concentration, for example, ${ }^{9} \mathrm{Be}$ in ITER. While a Monte Carlo model allowing one to convert from the number of counts in a given $\gamma$-ray peak to absolute flux can be set up (and is often developed), the concentration of impurities is often not well known and, sometimes, can only be estimated from models of impurity transport. In this case, the fast-ion density thus comes with a systematic uncertainty that is largely dominated by assumptions on the impurities.

The most advanced measurement parameter is the shape of the characteristic peaks associated with $\gamma$-ray emission. Figure 2 bottom shows the $4.44 \mathrm{MeV}$ peak shape from the ${ }^{9} \mathrm{Be}(\alpha, \mathrm{n}){ }^{12} \mathrm{C}^{*}$ reaction when the $\alpha$-particle energy distribution is that resulting from classical slowing down by multiple Coulomb collisions, as shown on
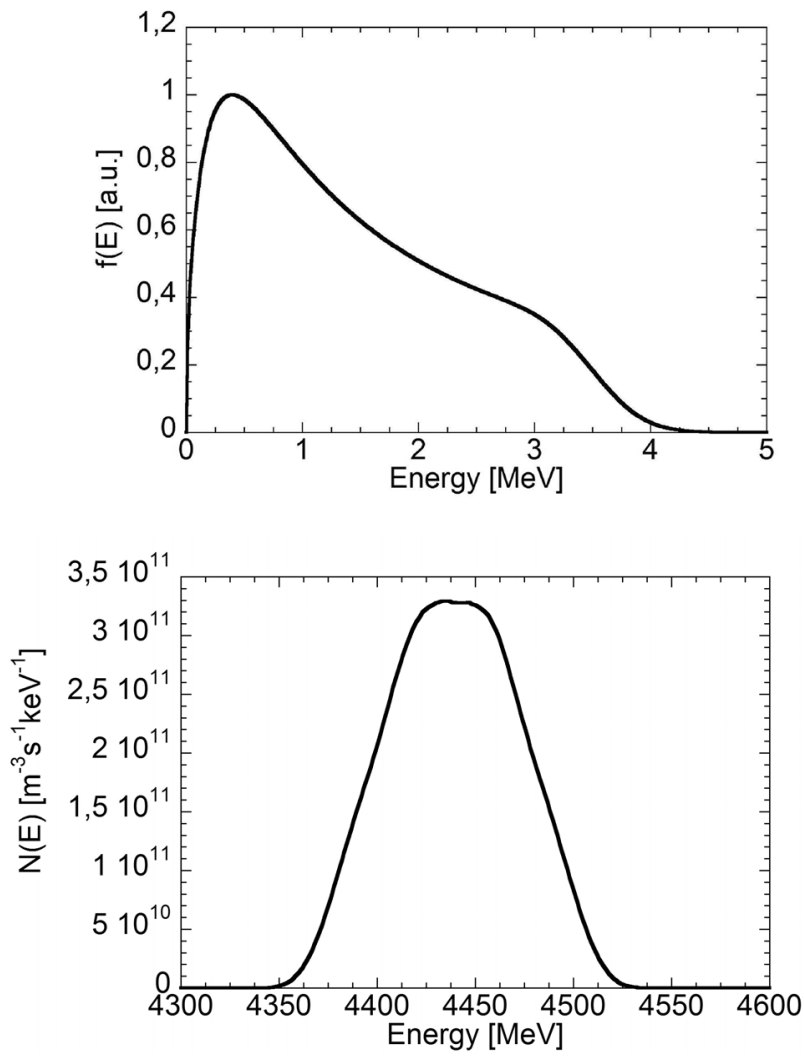

Fig. 2 a Classical $\alpha$-particle slowing-down distribution function in a plasma with temperature $T_{\mathrm{i}}=T_{\mathrm{e}}=20 \mathrm{keV}$, density $n=10^{20} \mathrm{~m}^{-3}$ and $1 \%$ beryllium concentration. b Spectrum of the $4.44 \mathrm{MeV}$ $\gamma$-ray peak from the ${ }^{9} \mathrm{Be}(\alpha, \mathrm{n}){ }^{12} \mathrm{C}^{*}$ reaction for the $\alpha$-particle distribution from a 
the top panel. The calculation was performed with the GENESIS code and shows that the peak has a trapezoidal shape with a full-width at half-maximum of about $100 \mathrm{keV}$. This kinematic broadening comes from distribution of projected velocities of the ${ }^{12} \mathrm{C}$ nucleus onto the line-of-sight of the detector which then leads to a distribution of Doppler-shifted $\gamma$-ray energies. The detailed relation between the peak shape and the underlying fast-ion distribution function is, however, often not straightforward. An important example is the $4.44 \mathrm{MeV}$ peak from ${ }^{9} \mathrm{Be}(\alpha, n){ }^{12} \mathrm{C}^{*}$. For this peak, the shape is most strongly influenced by $\alpha$-particles at $1.9,2.6$, and 4.0 $\mathrm{MeV}$ rather than by $\alpha$-particles at energies in between. The pitch angle distribution also influences the shape of the peak. In particular, as the application of weight function formalism (see Sect. 6) reveals (Salewski et al. 2015a), events in the center of the peak are mostly representative of co- and counter-passing ions, while counts at the high- and low-energy tails of the peak originate from trapped ions. Besides the ${ }^{9} \mathrm{Be}(\alpha, \mathrm{n}){ }^{12} \mathrm{C}^{*}$ reaction, similar modelling and analysis of the peak shape is nowadays often used to determine the energy distribution of deuterium (Eriksson et al. 2015), ${ }^{3} \mathrm{He}$ (Tardocchi et al. 2011), and ${ }^{4} \mathrm{He}$ Nocente et al. (2012b) in experiments based on ICRF heating to drive ions into the MeV range. At the highest level of detail, knowledge of the peak shape allows measurements of the fast-ion-velocity distribution function by velocity-space tomography (see Sect. 6) (Salewski et al. 2017).

As for neutrons, the spatial profile of $\gamma$-ray emission can also be measured, besides its spectrum. One interest is in this case to simultaneously determine the profiles of different energetic ions in the plasma. This is accomplished by integrating the signal in the energy bands associated with the corresponding $\gamma$-ray peaks and by separately determining the profile from counts in each of these bands. An example is the simultaneous determination of the profiles of deuterons and ${ }^{4} \mathrm{He}$ ions in experiments with ICRF heating at multiple harmonics (Kiptily et al. 2005). In this case, a very different profile was obtained by integrating data in the region around the 3.1 MeV and 4.44 MeV peaks from the ${ }^{12} \mathrm{C}(\mathrm{d}, \mathrm{p} \gamma){ }^{13} \mathrm{C}$ and ${ }^{9} \mathrm{Be}(\alpha, \mathrm{n}){ }^{12} \mathrm{C}^{*}$ reactions, respectively, which was explained by the different orbits associated with the ion energies that dominated the emission. Another application is to study the effect of instabilities driven by fast ions on the fast-ion population. A recent application is an experiment, where fast changes of the $\gamma$-ray profile were associated with a redistribution of fast ions determined by the onset of toroidicity-induced Alfvén eigenmodes (TAEs) in the plasma (Gassner et al. 2012).

\subsection{Instrumentation}

\section{Neutron measurements}

As neutrons are uncharged, their detection involves first the (full or partial) conversion of the incoming neutron energy to that of a charged particle, followed by its detection. In broad terms, we can distinguish between two families of detectors, depending on whether they feature spectroscopic capabilities or not. Since spectroscopy is the most interesting application for fast-ion studies, dedicated efforts have been primarily put in advancing that class of instruments with dedicated designs, which is the focus of this section. The non-spectroscopic instruments are also briefly 
discussed in this review [the interested reader can refer to the relatively old, but still valid, reference Jarvis (1994) for more details on this second type of detectors]. One of the main challenges of neutron measurements is that they must be undertaken in a high $\gamma$-ray background. Detectors must hence be capable to operate in such a background and to distinguish between neutron and $\gamma$-ray interactions. When the determination of the $f l u x$ is the scope of the measurements, fission chambers or activation foils are adopted. The insensitivity to $\gamma$-rays is obtained by operating fission chambers in the so-called 'Campbelling' mode, where the mean square voltage of the signal is measured which is very different for neutron and $\gamma$-ray interactions. Activation foils are per se insensitive to $\gamma$-rays as they cannot induce reactions that lead to the activation of the sample. An outstanding issue for both techniques is their absolute calibration (Batistoni et al. 2017), which is a laborious process. However, while the absolute calibration is needed to convert from the measured time trace to fusion power, it is not necessarily needed for fast-ion studies. One can still draw conclusions by comparing the measured time trace with that expected from calculations on a relative scale.

If the fluxes of 14 and $2.5 \mathrm{MeV}$ neutrons need to be separated, for example, for TBN studies in deuterium plasmas, detectors that selectively measure at $14 \mathrm{MeV}$ but not at $2.5 \mathrm{MeV}$ must be employed. Examples are silicon detectors, for example, at JET, where neutrons with an energy exceeding a threshold of $7 \mathrm{MeV}$ induce $(\mathrm{n}, \alpha)$ and $(\mathrm{n}, \mathrm{p})$ reactions leading to signals which are detected in pulse mode (Jarvis 1994). Alternatively, scintillating fibers have been used (Nishitani et al. 1996; Wurden et al. 1995). Such instruments are based on detecting the light produced by a proton using photomultipliers. 14 and $2.5 \mathrm{MeV}$ neutrons can be distinguished by a suitable selection of the energy threshold.

When spectroscopy is the main goal of the measurement, an essential parameter is the instrument response function. The response function that connects the quantity that is actually measured to the incoming neutron energy can be more or less complicated depending on the specific detection principle and detailed instrument design. For this reason, very different instruments are used that fall into two major groups: compact and non-compact spectrometers. Non-compact spectrometers have dimensions of a few meters but a relatively selective response function, which makes it possible to accurately measure the details of the neutron spectrum around the nominal $2.5 \mathrm{MeV}$ and $14 \mathrm{MeV}$ energies, of importance to derive the fast-ion energy distribution from data. The compact spectrometers have limited spectroscopic capabilities, but, being compact, can be arranged in cameras for profile measurements and are, therefore, preferred for neutron cameras. In recent years, compact detectors have also been proposed for detailed spectroscopy applications, most notably diamond detectors (Nocente et al. 2015b). However, the quality of the data they can provide does not yet fully compare to that of the dedicated spectrometers. Different instrumental designs are employed depending on the bulk plasma composition, i.e., D or DT.

For neutron profile measurements, a very popular detector is the liquid scintillator, which contains a liquid compound of carbon and hydrogen in the scintillation cell. The detection principle is based on nuclear elastic scattering reactions between incoming neutrons and the protons of the active material. From classical kinematics, 
a neutron with energy $E_{\mathrm{n}}$ that scatters off a proton at rest can leave a fraction between zero and its full energy $E_{\mathrm{n}}$ to the proton. The theoretical detector response to monoenergetic neutrons is a square, where the position of the edge represents the energy of the incoming neutron. In practice, however, no liquid scintillator has a response function as simple as a square, as complicating factors arise. One is the competing process of neutron scattering on carbon in the active cell. A second and most important complicating factor is that it is not the proton energy that is directly measured, but rather the light yield that protons induce as they are stopped in the scintillator material itself. This is often a non-linear function of the proton energy. A third complication comes from the finite energy resolution of the instrument, which broadens the edge. When all of these complicating factors are put together, the actual response of the instrument can depart significantly from the ideal square shape (see Fig. 3). A distinctive feature of liquid scintillators is their capability to discriminate between neutron and $\gamma$-rays by the pulse shape of the signal. This feature is especially important, as neutron-rich environments are always associated with an equally rich $\gamma$-ray background.

Even though some neutron spectroscopy with liquid scintillators has been attempted (Zimbal et al. 2004), these detectors are more often used as counters in profile measurements. In this application, a number of liquid scintillators are put at the end of collimated lines-of-sight to measure the neutron emissivity along each chord (see Fig. 2.2) (Fig. 4).

Tomographic inversion techniques (Craciunescu et al. 2009) are used to reconstruct the local emissivity profile from line-integrated measurements. Experimentally, the spectroscopic capability of the each detector is used to carefully set individual thresholds. Only events that fall above the threshold are recorded in pulse mode. The amplitude of the threshold is chosen as a compromise between the requirement to maximise the counting rate capability (and hence time resolution)

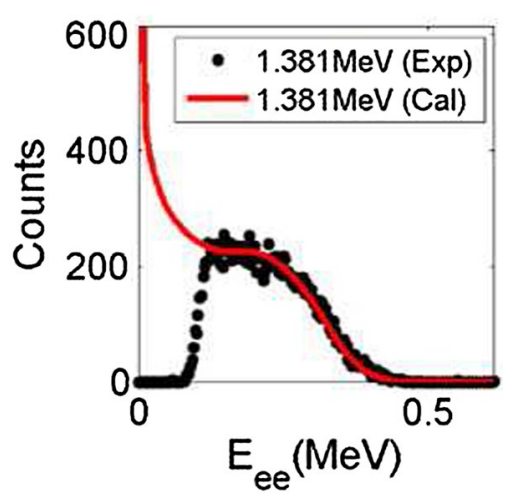

Fig. 3 Pulse-height spectrum measured by an EJ301 liquid scintillator detector exposed to monoenergetic $1.381 \mathrm{MeV}$ neutrons. The $x$-axis shows the equivalent electron energy $E_{\mathrm{ee}}$, i.e., the proton energy once the non-linear light yield is taken into account and expressed in terms of electron energies that would give the same light yield. The red curve is the result of a simulation of the expected response. A low-energy threshold is used in the measurement to avoid low-amplitude noise 

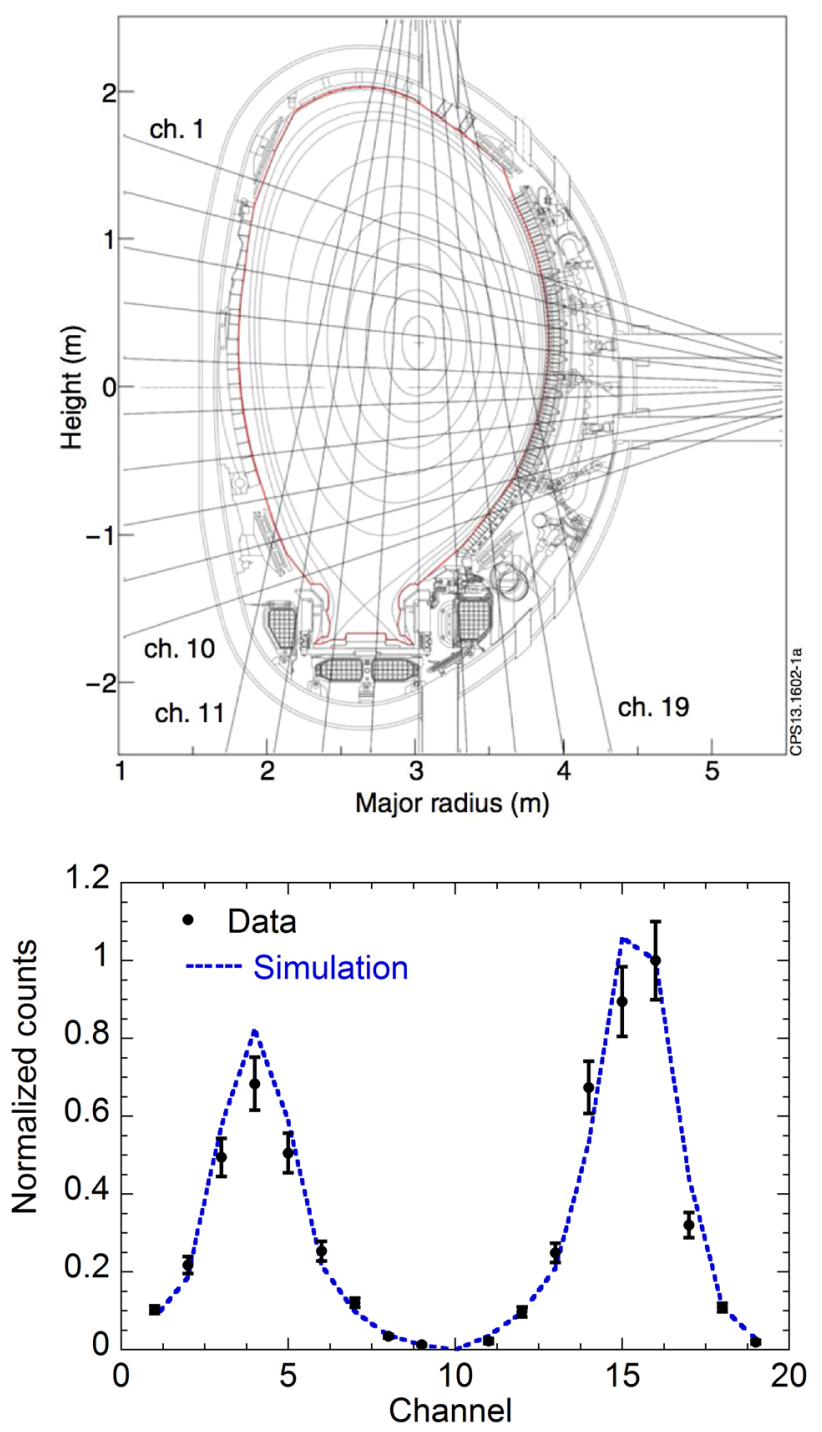

Fig. 4 a Sketch of the two JET neutron cameras (vertical and horizontal). The vertical and horizontal lines are the observation chords of each channel of the two cameras. The detectors, not shown in this picture, are put at some distance (about $1.4 \mathrm{~m}$ from the first wall at JET) along each chord. The figure is taken from Nocente et al. (2014). b Examples of data from the JET neutron camera and in a trace tritium plasma. Counts measured by each channel of the horizontal (ch. 1-10) and vertical (ch. 11-19) neutron camera are shown with error bars. The dashed line is a simulation based on calculations of the fast-ion distribution function with the TRANSP code. The plasma was heated by NBI of deuterons and tritons. The figure is taken from Nocente et al. (2014)

of the measurements and the need to minimize the extent of the $\gamma$-rays and scattered neutrons in the recorded signal. 
These are neutrons that have lost energy by interactions with the tokamak structures (divertor, first wall etc.) as they travel along their path from the plasma to the instrument (see Sect. 2.3). The extent of scattered neutrons can be especially important for lines-of-sight that do not explore the plasma core. The stability of the threshold and signal pile-up must also be considered. In practice, the dimensions of the detector are chosen, so that the counting rate does not exceed about $500 \mathrm{kHz}$. For higher counting rates, for example, those found in DT plasmas, different detectors are preferred. A popular choice is Bicron BC418 (Jarvis 1994), which is a plastic scintillator detector with significantly less efficiency to $\gamma$-ray detection. More recently, synthetic diamond detectors are emerging as a promising technology, especially in view of ITER (Cazzaniga et al. 2014a, b). Neutron cameras have been developed for the major tokamak experiments, for example, JET (Jarvis 1994), TFTR (Roquemore et al. 1990), JT-60U (Ishikawa et al. 2002), EAST (Zhong et al. 2016), and the LHD stellarator (Ogawa et al. 2014).

When the goal is to measure the high-energy tails of the neutron spectrum, such as needed to study the energy distribution of the fast ions, an instrument that provides a significantly more selective response function, improved stability, counting rate capability, and higher dynamic range than liquid scintillators is mandatory. To this end, two different techniques are most popular: the time-of-flight (TOF) for deuterium plasmas and the magnetic proton recoil (MPR) for deuterium-tritium plasmas. In the TOF technique, energy is measured by the TOF of neutrons traveling a known distance. They scatter first on a set of scintillator detectors and are then detected again on a second umbrella of detectors (see Fig. 5, top). The umbrella covers the so-called sphere of constant TOF (Legge and Van der Merwe 1968), so that the TOF of the scattered neutrons is a measurement of the energy of the incoming neutrons. Operationally, all the interactions that occur on the two sets of scintillators are recorded by a free streaming digitizer. For each event at a time $t_{\mathrm{TOF}}$ on the second set of scintillators, all events on the first set of scintillators that occurred at a time $t_{\mathrm{TOF}} \pm \Delta t(\Delta t \approx 200 \mathrm{~ns}$, typically) are used to build the time-of-flight spectrum. The upper limit to the counting rate at which the TOF technique can be applied without paralyzing the detector is set by random coincidences. These are events that appear as coincident within the instrument time acceptance window, but come instead from the background, i.e., they do not correspond to a neutron that actually travelled from the first to the second set of scintillators within the acceptance time window. The rate of random coincidences scales as the square of the neutron rate which limits the maximum counting rate capability of the instrument to up to $500 \mathrm{kHz}$. This hampers the applications of the TOF technique for DT plasmas, where MPR detectors are preferable. Detectors based on TOF are the TOFOR neutron spectrometer at JET (Gatu Johnson et al. 2008) and the TOFED instrument at EAST (Zhang et al. 2014a, b). Fast-ion applications include studies of fast ions produced by NBI (Hellesen et al. 2010) and ICRF heating (Eriksson et al. 2015; Hellesen et al. 2013), including the effects of sawteeth and TAEs on fast ions in the MeV range (Gassner et al. 2012; Hellesen et al. 2010).

The MPR technique is based on a different detection method. In this instrument, neutrons from the plasma scatter in a polyethylene target and produce recoil protons. The recoil protons are momentum analyzed using a large magnet bending their 

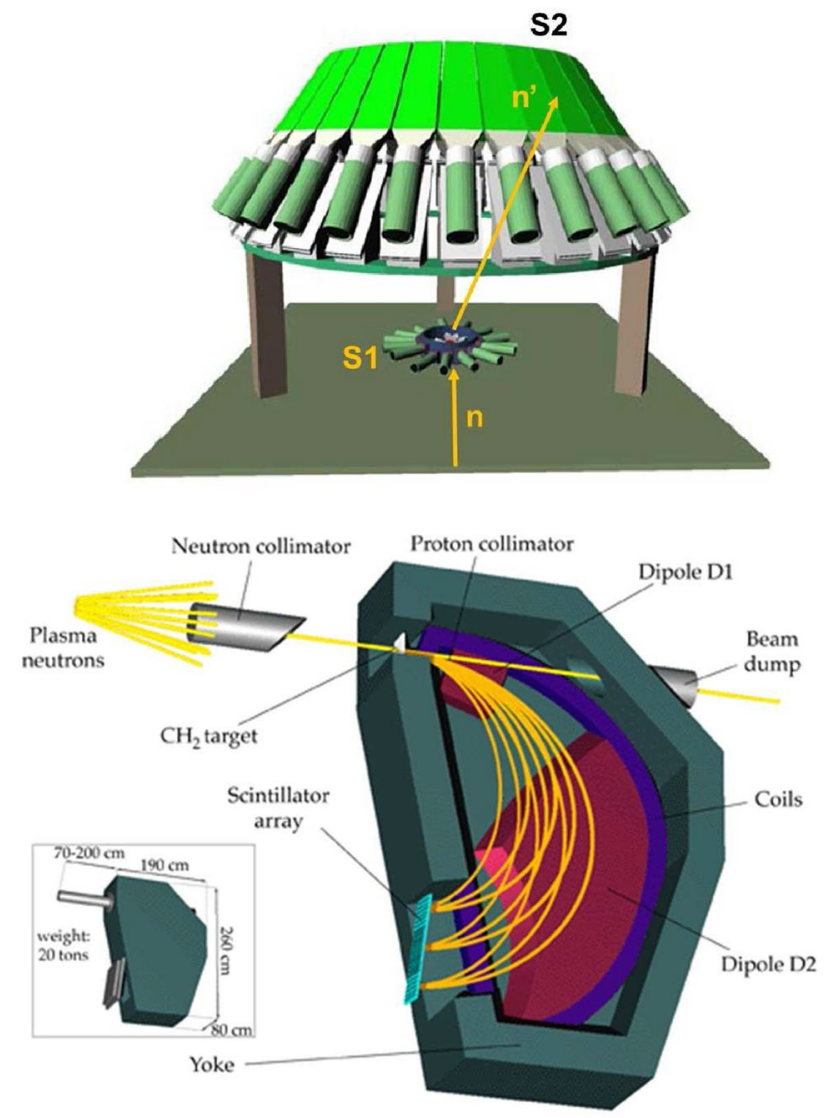

Fig. 5 a Schematics of the TOF technique for spectral measurements of neutrons in D plasmas. The neutron beam impinges on a stack of scintillators (S1). Scattered neutrons are recorded by an umbrella of stop detectors (S2). The TOF between scattering in S1 and detection in S2 gives the incoming neutron energy. b Schematics of the magnetic proton recoil technique. Elastic scattering of a neutron beam on a polyethylene target produces recoil protons, which are dispersed to different energy-dependent positions on a scintillator array in a magnetic field. Reproduced with kind permission of Societa Italiana di Fisica. Copyright (C) Societa Italiana di Fisica. Reference: M. Nocente, "Fast-ion measurements with neutron and $\gamma$-ray spectroscopy in thermonuclear plasmas: recent results and future prospects", Nuovo Cimento C, 39 (2016) 289 DOI: 10.1393/ncc/i2016-16289-6

trajectories to different impact positions on a scintillator array (see Fig. 5, bottom). The energy spectrum of incoming neutrons is thus transformed into a position histogram on the array. The thickness of the target is chosen as a compromise between the energy loss of protons within the target and the detection efficiency. The main advantage of this technique is its capability to sustain $\mathrm{MHz}$ counting rates. Therefore, MPR detectors are especially suitable for applications in high-performance DT plasmas, where the extent of random coincidences from the background would be too high for the TOF technique to work. Applications of the MPR to D plasmas are more difficult, mostly because practical values of the polyethylene thickness 
result in a detection efficiency of about $10^{-4}$, which is two orders of magnitude worse than about $10^{-2}$ of the TOF technique. The MPR principle is employed by the MPRu spectrometer at JET (Sjostrand et al. 2006). An example of a neutron spectrum measured with this instrument in a trace tritium experiment at JET is shown in Fig. 6. Fast-ion applications of the technique include the important assessment of classical $\alpha$-particle slowing down in the JET 1997 DT experiments by observations of the corresponding knock-on component in the spectrum (Kaellne et al. 2000) (see Sect. 2.1). More traditional applications are for studies of NBI ion transport in trace tritium plasmas (Nocente et al. 2014) as well as the acceleration of tritium ions by different ICRF schemes (Tardocchi et al. 2002). Unlike TOF instruments, the use of MPR for extended physics studies has been fairly limited up to now, mostly because of the small number of tritium studies in present tokamak experiments. A common feature of TOF and MPR is that they provide an almost one-to-one correspondence between neutron energy and the quantity that is actually measured (TOF or position), which greatly simplifies the analysis and adds to the stability of the detector. Still, a detailed knowledge of the instrument response function is mandatory to extract quantitative information (Jacobsen et al. 2017).

Gamma-ray measurements The detection of $\gamma$-rays is comparably simpler than the detection of neutrons and involves the conversion of the radiation energy to energy of one or more electrons which are subsequently stopped in the detector material. The conversion can proceed via the photoelectric, Compton and pair production processes or, in most cases, a combination of them (Knoll 2010). The relative importance of the three processes depends on the detector size and detailed geometry. Figure 7 shows a typical response function in the case of a

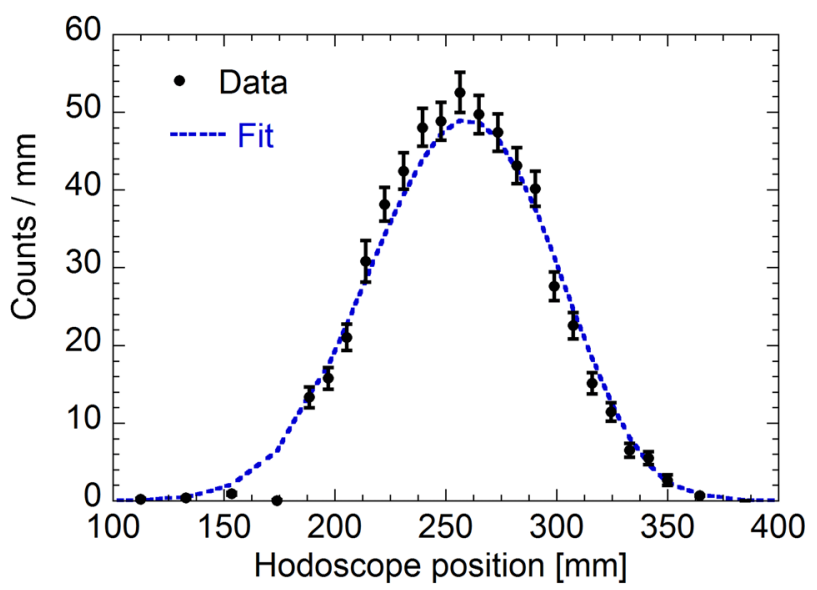

Fig. 6 Neutron spectrum measured by the MPRu magnetic proton recoil spectrometer at JET in a discharge of the trace tritium experiment with deuterium and tritium NBI. The spectrum is centered at 14 $\mathrm{MeV}$, corresponding to the position at $250 \mathrm{~mm}$ on the hodoscope, and has a shape determined by reactions between thermal and fast beam ions and their combinations. The solid line is a fit to measured data based on a model of the neutron emission for this discharge. The figure is taken from Nocente et al. (2014) 


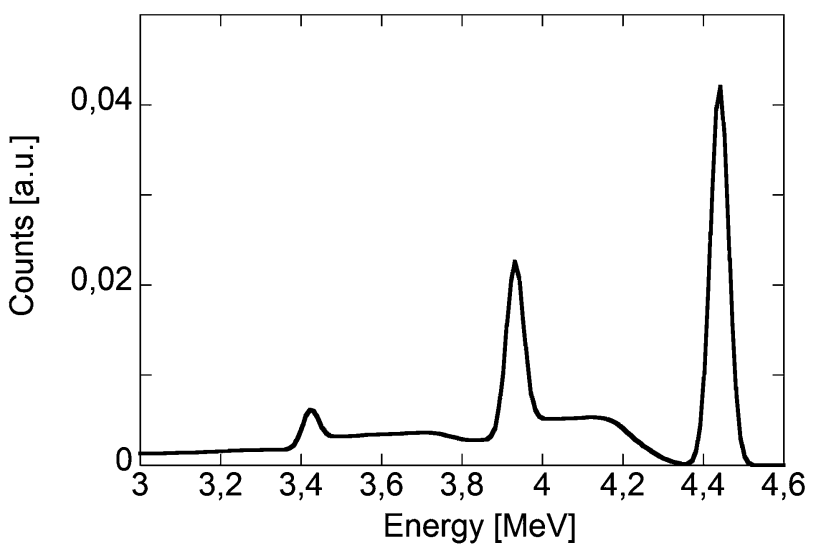

Fig. 7 MCNP simulation of the $\gamma$-ray spectrum recorded by a $3^{\prime \prime} \times 6^{\prime \prime}$ (diameter $\times$ height) $\operatorname{LaBr}_{3}$ detector when exposed to $4.44 \mathrm{MeV} \gamma$-rays. The spectrum shows the full-energy peak at $4.44 \mathrm{MeV}$, as well as the single and double escape peaks (Knoll 2010) at 4.44-0.511 and 4.44-1.022 MeV, respectively, resulting from pair production in the detector. The continuum is due to Compton interactions. An energy resolution of $3.1 \%$ at the $662 \mathrm{keV}$ line from ${ }^{137} \mathrm{Cs}$ is assumed

$E_{0}=4.44 \mathrm{MeV} \gamma$-ray from the ${ }^{9} \mathrm{Be}(\alpha, \mathrm{n}){ }^{12} \mathrm{C}^{*}$ reaction that impinges on a $3^{\prime \prime} \times 6^{\prime \prime}$ $\mathrm{LaBr}_{3}$ crystal. The signature of the different concurring processes is seen as the appearance of peaks due to the photoelectric effect (at $E_{0}$ ) and pair production (at $E_{0}-0.511 \mathrm{MeV}$ and $E_{0}-1.022 \mathrm{MeV}$ ). These sit on a continuous structure that comes from Compton interactions. In addition, there can then be an instrumental broadening of each channel of the spectrum, the magnitude of which mostly depends on the detector material. The broadening of the full-energy peak from a calibration source with a well-defined energy (for example, $662 \mathrm{keV}$ from ${ }^{137} \mathrm{Cs}$ ) defines the instrumental resolution at that energy.

Unlike in neutron spectroscopy, it is often not the overall shape of the spectrum that is used for fast-ion studies by $\gamma$-ray spectroscopy, but only the full-energy peak. As mentioned in Sect. 2.1, experimentally the detection of fast ions by $\gamma$-ray emission consists of identifying the reactions that can lead to the measured peaks, of analyzing their intensities and ratios and, in the most advanced applications, of measuring their detailed shapes. For this reason, large detectors (a few inches diameter by a few inches length) that maximise the probability of a photoelectric interaction are chosen. In terms of detector type, the most popular choice are inorganic scintillators. Initially, $\mathrm{NaI}(\mathrm{Tl})$ and BGO were used (Kiptily et al. 2002, 2006), mostly because of their large availability in the field of applied nuclear physics and since they provided a reasonable compromise between detection efficiency and energy resolution. Nowadays, new, improved inorganic scintillators have emerged. A notable example is $\mathrm{LaBr}_{3}$ (Nocente et al. 2010b), which offers about a factor two better energy resolution than $\mathrm{NaI}(3.1 \%$ compared to $7 \%$ at $662 \mathrm{keV})$ and a decay time as fast as $30 \mathrm{~ns}$, which opens up to $\gamma$-ray spectroscopy at MHz counting rates (Nocente et al. $2013 b$ ). Such counting rates are mandatory for applications in high-power DT plasmas. Since its installation at JET, $\mathrm{LaBr}_{3}$ with preferred dimensions of $3^{\prime \prime} \times 6^{\prime \prime}$ and a 
$\sim 30 \%$ full-energy peak efficiency has now become the reference choice also in view of ITER (Nocente et al. 2017; Chugunov et al. 2011).

When analysis of the fine peak shape is the aim of the measurement, a detector which offers a virtually zero line instrumental broadening is desired. High-purity germanium (HpGe) detectors (Tardocchi et al. 2011), which are based on collecting the large number of electron-hole pairs generated by the interaction of $\gamma$-rays with the active material, are here the natural choice. An instrumental broadening of about $0.1 \mathrm{keV}$ is easily achievable for emission lines in the $\mathrm{MeV}$ range with these detectors. This instrumental broadening is negligible compared with typical values of the Doppler broadening of about $100 \mathrm{keV}$ and makes the measured line shape representative of the fast-ion motion only. An example of a $\gamma$-ray spectrum measured in an ICRF-heating experiment at JET with HpGe is shown in Fig. 8.

The disadvantages of detectors based on $\mathrm{HpGe}$ compared with $\mathrm{LaBr}_{3}$-based detectors are that they must be cooled, they have about 4-5 times less detection efficiency for practical detector dimensions, and even though they were demonstrated to work up to about $1 \mathrm{MHz}$, they have a limited throughput as the counting rate approaches some hundreds of $\mathrm{kHz}$ (VanDevender et al. 2014). In practice, in modern installations, most often both $\mathrm{LaBr}_{3}$ and $\mathrm{HpGe}$ are available on the same line-ofsight, and a selection on which detector to use is made on a case-by-case basis.

Cylindrical detectors with a diameter and length of a few inches as those described so far can also be used for $\gamma$-ray profile measurements. This is the approach envisaged for ITER (Nocente et al. 2017). However, in existing tokamaks, for example, at JET, $\gamma$-ray detectors were developed at a later stage than those for

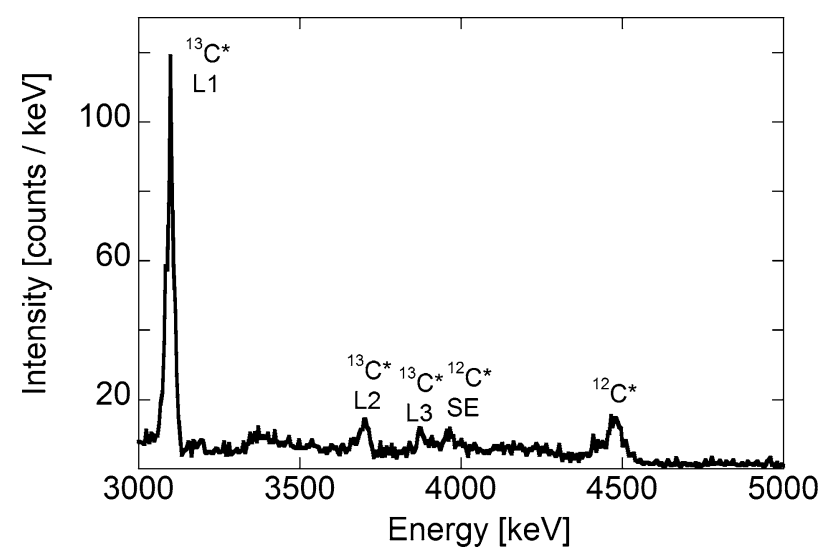

Fig. 8 Gamma-ray spectrum measured with a HpGe detector in a discharge with third harmonic ICRF heating of ${ }^{4} \mathrm{He}$ ions at JET with a carbon wall. The full-energy and single-escape peaks produced by $\gamma$-rays born in the ${ }^{9} \mathrm{Be}\left({ }^{4} \mathrm{He}, \mathrm{n}\right){ }^{12} \mathrm{C}$ reaction between fast ${ }^{4} \mathrm{He}$ ions and ${ }^{9} \mathrm{Be}$ impurities injected with overnight evaporation are seen. There are also full-energy peaks produced by the de-excitation of the first, second, and third excited states of ${ }^{13} \mathrm{C}$ born from the ${ }^{12} \mathrm{C}(\mathrm{d}, \mathrm{p}){ }^{13} \mathrm{C}$ reaction between fast deuterons and ${ }^{12} \mathrm{C}$ impurities. All peaks feature an experimental full-width at half-maximum broadening of about $50 \mathrm{keV}$. This exceeds by far the expected instrumental line broadening of $<2 \mathrm{keV}$ and unambiguously reveal the contribution of the Doppler broadening to the measurements. The figure is taken from Nocente et al. (2012b) 
neutrons. $\gamma$-ray profile capabilities were added to an already existing neutron camera. Because of this, the space limitations are important, since the photomultiplier tubes of the scintillators must be shielded against the magnetic field, which is fairly large at the neutron camera location. This would ideally require a combination of mu metal and a few centimeters of soft iron, but this is not possible in practice due to the space limitation. The actual implementation is, therefore, based on an alternative sensor to photomultiplier tubes which does not require a magnetic shielding and ensures compact dimensions. At JET, for instance, CsI(Tl) scintillators are coupled to photodiodes. They fit a cylindrical capsule of about $3 \mathrm{~cm} \times 3 \mathrm{~cm}$, diameter $\times$ height. Measurements of the $\gamma$-ray emission profile were successfully demonstrated in D plasmas (Kiptily et al. 2006). A drawback of the present setup is its poor energy resolution, which makes it impossible to clearly observe characteristic peaks in the spectrum during a single discharge. This limits the availability of profile measurements to low neutron-yield discharges, where the neutron background in the detectors does not dominate over the signal. Another limitation is the slow decay time of $\mathrm{CsI}(\mathrm{Tl})$, about $1 \mu \mathrm{s}$, which implies a maximum counting rate in the range $<100 \mathrm{kHz}$. Energy-band selection is also constrained to four intervals only. It can be an issue to clearly distinguish between signal and neutron-induced background in some cases.

To overcome this limitation, new detectors have recently been developed. They make use of silicon photomultipliers and $\mathrm{LaBr}_{3}$ as an upgrade of $\mathrm{CsI}(\mathrm{Tl})$ and photodiodes. The advantages are an energy resolution comparable to that obtained with photomultiplier tubes, i.e., between 4 and $5 \%$ at the $662 \mathrm{keV}$ line (Rigamonti et al. 2016), and a significantly faster pulse width of about $100 \mathrm{~ns}$, which opens up to applications in high neutron-yield discharges at $\mathrm{MHz}$ counting rates (Nocente et al. 2016). The use of a dedicated fully digital acquisition system (Fernandes et al. 2014), together with the good energy resolution and time response of the new detectors, make it possible to precisely select only the energy bands associated with the specific fast-ion reactions of interest, as well as to eliminate the interference of neutron-induced events in the spectrum by subtraction of the background in the vicinity of the emission peaks. This is mandatory to allow for measurements of the $\gamma$-ray profile in high-performance D and DT plasmas.

\subsection{Prospects for ITER}

The development of suitable collimators is one of the main experimental challenges for neutron and $\gamma$-ray diagnostics. The collimation of neutrons and $\gamma$-rays is more difficult than for charged particles. A careful shielding has to be designed. Neutron cameras, for example, practically consist of a block of concrete (say, with a weight of some tons), where conical holes with a length of few meters and a diameter of few $\mathrm{cm}$ at the detector position are drilled. The exact dimensions of the concrete shielding are carefully studied by means of lengthy simulations of neutron transport from the plasma to the detector. Almost always detailed Monte Carlo codes such as MCNP (Monte Carlo Code Group 0000) are used. The actual geometry and materials of the tokamak are implemented with a high degree of fidelity. The aim of the design is to make sure that the fraction of the plasma volume seen by the diagnostic 
is well defined. For example, in neutron cameras, the shielding is studied, so that each detector measures the emission as closely as possible only along a chord (see the diagram in Fig. 2.2). However, no material is a perfect neutron absorber. Neutrons from plasma volumes not intended to be seen by the diagnostics also reach the detector. These so-called scattered neutrons, however, have significantly lower energy than 2.5 or $14 \mathrm{MeV}$, as they are moderated by the traversed material. They can often be discriminated from the 2.5 or $14 \mathrm{MeV}$ neutrons by setting a suitable energy threshold on the detectors. The discrimination capability between direct (uncollided) neutrons and collided neutrons depends on the quality of the shielding design and the stability and response function of the instrument. Most often, besides concrete, high-efficiency thermal neutron absorbers (boron or lithium) are included in the design, as well as effective hydrogen-rich moderators, for example, the widely used polyethylene or, in some cases, water. A side effect of neutron moderation and capture is the production of background $\gamma$-rays. These are born from unavoidable spontaneous nuclear reactions between neutrons and shielding materials or from inelastic neutron scattering. For this reason, a careful simulation of the background $\gamma$-ray generation by neutrons and its transport is also an important task. $\gamma$-ray absorbers, most popularly lead or iron, are included in the design and generally placed in vicinity of the detector. An additional difficulty comes from the possible further generation of $\gamma$-rays by these absorbers when they are exposed to neutrons. An iterative approach that proceeds by trial and error is often adopted in the simulations. In case of $\gamma$-ray measurements, specific neutron attenuators must also be placed in front of the detectors to limit the background produced by the interactions of the direct neutrons with the bulk material of the instrument (Cazzaniga et al. 2013, 2015; Fehrenbacher et al. 1996). Here, the preferred choices are polyethylene for D plasmas and LiH (Chugunov et al. 2008) for DT plasmas.

Practical constraints that limit the design, for both neutron and $\gamma$-ray measurements, are the weight and the space. Typically, the weight has to be limited to a few tons, which constrains the amount of concrete that can be used. Space is also an issue, as a large number of other diagnostic systems must also be deployed in a tokamak. In practice, only one horizontal and one vertical neutron $/ \gamma$-ray camera (see Fig. 2.2) with about 20 detectors in total, a few high-resolution $\gamma$-ray spectrometers and one D and one DT high-resolution neutron spectrometers are possible at most.

From the physics point of view, a difficulty in the interpretation of data is the indirect relation between the fast ions and the spectrum and spatial profile of nuclear radiation. The most common approach is to start from a model of the fast-ion distribution function and use it to calculate the expected, spatially resolved spectrum of nuclear emission by dedicated Monte Carlo codes (Nocente 2012; Eriksson et al. 2016), as well as the specific signals seen by each instrument. One must account for the details of its response function (which must be carefully simulated and experimentally validated) and radiation transport from the plasma to the detector, which includes an evaluation of the extent of the background (gamma rays and scattered neutrons). A comparison between the synthetic signal and the actual measurement reveals whether the input fast-ion model is compatible with the data or must be improved. An alternative is the tomographic inversion of the measured spectra by velocity-space tomography (Sect. 6). 
In general, neutron emission simulations are easier than $\gamma$-ray emission simulations, as there are only two fusion reactions that produce neutrons, i.e., $d(d, n)^{3} \mathrm{He}$ and $t(d, n)^{4} H e$. The cross sections are well established. $\gamma$-ray emission simulations are more challenging. There are a large number of reactions that lead to $\gamma$-ray emission. This opens up to the simultaneous observation of different fast ions at the same time, rather than only deuterons and tritons as for neutrons. However, this also makes the simulation more complex. An additional difficulty comes from the relatively limited availability of good cross-sectional data for a number of reactions. The most simple parameter to simulate is the intensity of the emission, as this just requires knowledge of the total cross section. Even in this case, however, measurements of the differential cross section at one specific emission angle (termed the excitation function) are sometimes available only and little is known about the full differential cross section, for example, its anisotropy as a function of energy [see the discussion of Nocente et al. (2012b)]. When it is so, the total cross section can be assumed to be given by $4 \pi$ times the excitation function, but this introduces a systematic uncertainty which might have an impact on the plasma parameters that are derived from the measurements. Besides, some of the emission peaks can depend on the de-excitations of multiple excited states by cascade transitions (Tardocchi et al. 2011; Proverbio et al. 2010) and knowledge of the differential cross section to populate each of the excited states would be required for a full simulation. A similar argument applies to peak Doppler-broadening studies, as the detailed line shape is even more sensitive to the anisotropy of the differential cross section. Presently, missing cross-sectional data could be measured at a large number of existing nuclear facilities, once a list of the most important and intense reactions for fast-ion diagnostic applications has been compiled based on present experience. For example, cross sections for reactions between fast ${ }^{3} \mathrm{He}$ and deuterium on ${ }^{9} \mathrm{Be}$ impurities have presently important gaps and would be required for studies of fast ions in ICRF experiments at ITER.

The absolute intensity of the $\gamma$-ray emission depends not only on the cross section and the fast-ion distribution function but also on the impurity concentration which is often not well known. However, the ratio of characteristic peaks from the same reaction as well as the peak Doppler broadening are independent of the impurity concentration and are related to the fast-ion distribution function only. In practice, complications arise. For some reactions, the Doppler broadening and peak ratio tend to saturate at high fast-ion energies (say, at a few hundreds $\mathrm{keV}$ tail temperatures for ICRF-heating scenarios) and the absolute intensity of the emission must be also taken into account to extract quantitative diagnostic information at these energies. Here, a fundamental advantage comes from the fact that the absolute intensity often changes as a power law of the fast-ion temperature, with typical exponents significantly larger than one, whereas it depends only linearly on the impurity concentration. An uncertainty even up to a factor of 3-4 on the impurity concentration is, therefore, of little practical relevance to constrain the fast-ion distribution function.

If neutron and $\gamma$-ray diagnostics certainly present experimental and interpretation challenges, they also provide essential information about the core plasma in high-power tokamaks, such as JET and even more ITER. The main advantage is the increasing (by orders of magnitude) neutron and $\gamma$-ray fluxes in large, 
high-performance devices, which implies a largely improved signal-to-noise ratio (SNR) and significantly lower integration time to obtain data with acceptable statistics compared to most present experiments. At ITER, for example, first calculations show that nuclear radiation measurements with a time resolution of relevance to perform fast-ion slowing-down studies-or to track fast-ion profile changes as a result of MHD instabilities - are within reach.

The implementation of nuclear diagnostics at ITER is not entirely different from the present experience at JET. The instruments are placed at some meters from the center of the machine, in some cases behind a bio-shield, where access for system maintenance and detector replacement, albeit seldom, can be envisaged. The extremely harsh plasma conditions of ITER, i.e., an increase of the particle and nuclear heating by orders of magnitude compared to present devices make the implementation of most fast-ion diagnostics extremely challenging, but are of no relevance for neutron and $\gamma$-ray diagnostics. Intrinsic limitations due to the combination of an increased background and significantly smaller cross sections, which plague, for example, FIDA in large tokamaks, do not apply. Neutrons and $\gamma$-rays carry information about the core of a tokamak plasma, including confined fast ions, and are therefore essential to understand reactor-relevant plasmas.

\section{Neutral particle analyzers and fast-ion D-alpha spectroscopy}

The transfer of electrons from donor neutrals to ions, called charge exchange, has been detected more than 100 years ago (as stated by Allison 1958) and builds the basis for two important fast-ion diagnostics in fusion devices: NPA and FIDA, which we discuss in this section. NPAs measure the flux of neutralized hydrogen isotopes onto a detector. They have a long tradition in fusion research, because central ion temperatures could be obtained from the measured energy distribution of neutralized particles during early fusion experiments (Afrosimov 1961). These passive measurements were possible, because considerable densities of donor neutrals were present in the plasma due to the low temperatures and the low densities. With increasing plasma performance, however, passive charge-exchange measurements suffer from poor SNR, since almost all particles in the plasma core are fully ionized. In contrast, active charge-exchange measurements, based on donor neutrals injected by NBIs, became possible thanks to the development of NBIs in the 1970s (Speth 1989). Here, viewing geometries that cross a given NBI line allow measurements with good spatial resolution. In particular, spectroscopic measurements have become the main diagnostic in many fusion devices to determine the impurity ion temperature, rotation, and density (Isler 1977; Fonck 1985). Moreover, active NPA measurements carry information on the fast-ion distribution function. In addition, the analysis of Doppler-shifted charge-exchange radiation of suprathermal particles became possible in recent years due to improvements of the diagnostic equipment, in particular CCD cameras.

In the following, first, the charge-exchange process is discussed in Sect. 3.1. Then, details on the NPA measurement are given in Sect. 3.2, followed by a presentation of FIDA spectroscopy in Sect. 3.3. 


\subsection{Physics principle}

In a charge-exchange process, an energetic or thermal ion in the plasma catches an electron from a donor neutral. The momentum exchange is negligible, because the electron mass is significantly lower than the ion mass. Thus, the analysis of particles after charge-exchange yields information on the former confined ions. The reaction for hydrogen isotopes can be expressed as

$$
X^{+}+X(n) \rightarrow X(m)+X^{+} .
$$

Here, $X^{+}$is the hydrogen isotope ion, $X(n)$ is a hydrogen donor neutral in atomic state $n$, and $X(m)$ is the resulting neutralized ion in atomic state $m$. The cross section for this process depends strongly on the initial and final atomic states and on the relative collision energy.

The cross sections are plotted in Fig. 9 as a function of the collision energy per atomic mass unit. They strongly decrease above about $30 \mathrm{keV} / \mathrm{amu}$ (60 keV for Deuterium). This already illustrates that NPA and FIDA measurements are not suitable to detect fast ions in the $\mathrm{MeV}$ range.

The charge-exchange process is exploited in active and passive diagnostics. On the one hand, the donor neutrals can be background neutrals mainly coming from the walls, which will yield passive charge-exchange signals. On the other hand, active charge-exchange signals are expected from the interaction of fast ions with neutrals present in NBI paths, which consist of injected neutrals and halo neutrals. Halo neutrals have thermal energies and originate from charge-exchange reactions between the injected NBI neutrals and thermal hydrogen ions (or hydrogen isotopes). In addition, halo neutrals are formed by the charge-exchange process between thermal ions and other halo neutrals. The cloud of halo neutrals has a larger spatial extent than that of injected NBI neutrals. Moreover, its contribution to the active chargeexchange signals can even exceed the contribution from the directly injected neutrals

Fig. 9 Charge-exchange cross sections between hydrogen atoms at various excited states and hydrogen ions

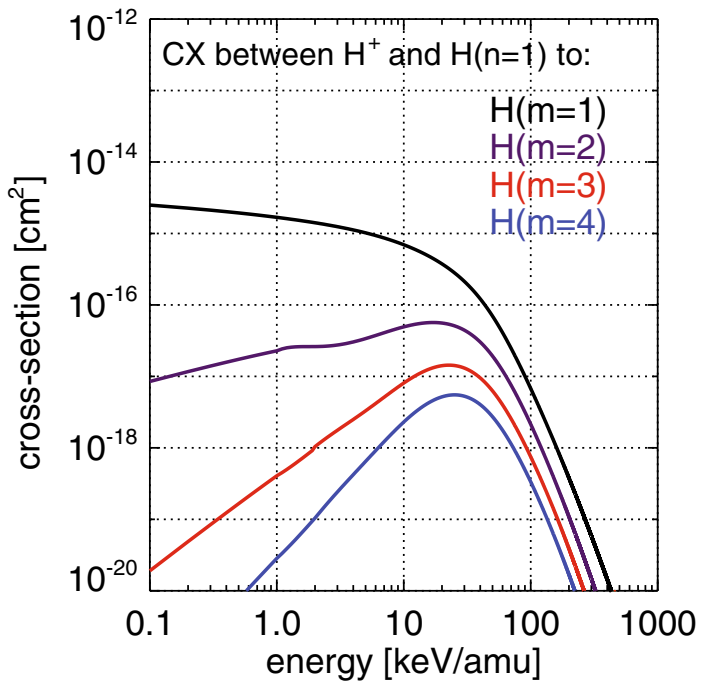


(depending on the plasma temperature and the NBI injection energy). It is, thus, of importance to consider the cloud of halo neutrals when analyzing active chargeexchange signals (FIDA or NPA) (Takafumi et al. 2010).

\subsection{NPA}

After the charge-exchange reaction, the hydrogen isotopes become neutralized and hence move on straight paths through the plasma. Along their path, the neutral particles might get re-ionized by electron impact ionization, ion-impact ionization, or charge-exchange and remain in the plasma. Alternatively, they leave the plasma and hit the walls. This process, called charge-exchange losses, can significantly reduce the plasma energy when large neutral densities are present (Geiger et al. 2017). These charge-exchange losses can be detected by NPAs which allows the analysis of the fast-ion distribution function.

The technical details of NPAs are well described in Medley et al. (2008). In this paper, we focus on the interpretation of the NPA signal. NPA detectors typically have a very good energy resolution and can often resolve isotopes. In addition, the SNR of the measurement is very good. The SNR is mainly limited by the detector characteristics and only to a small degree by additional contributions induced by the plasma. Only neutrons or $\gamma$-rays might additionally affect the measurement, while, e.g., FIDA and CTS measurements are strongly affected by different kinds of plasma radiation. Thus even low fluxes of neutralized fast ions provide valuable information. The observed signal can span several orders of magnitude (see Fig. 10). Another highlight of NPA diagnostics is the well-defined viewing geometry which defines the velocity vector range of the observed particles very well.

However, NPA measurements are often dominated by passive signals from charge-exchange reactions between the fast and thermal ions in the plasma and donor neutrals from the walls. The passive signal contains information on the fastion distribution and ion temperature, but is difficult to interpret: The density profile of donor neutrals from the walls is typically not well known, which makes the determination of the neutralization position of the detected neutrals challenging. This limits not only the spatial resolution of the measurement, but also the velocity-space

Fig. 10 Measurement of the NPA diagnostic at TCV, showing that the signal spans over six orders of magnitude. The figure is taken from Karpushov et al. (2006)

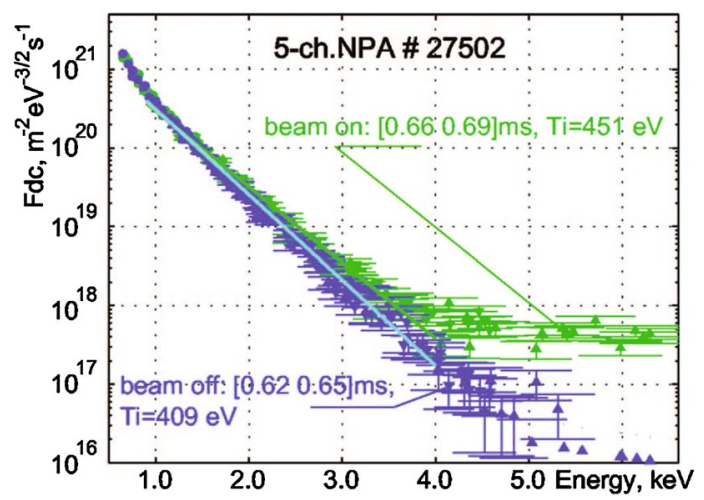

Springer 


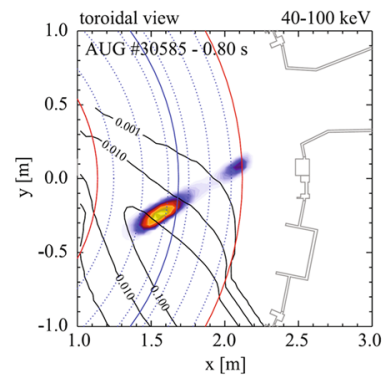

(a)

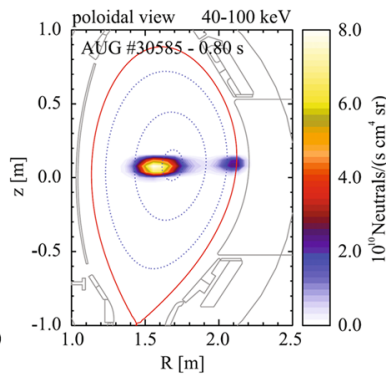

(b)

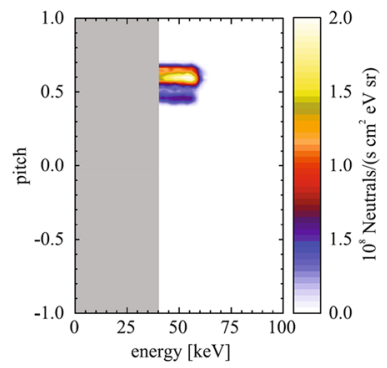

(c)

Fig. 11 Toroidal (a) and a poloidal (b) projections of simulated birth locations of neutrals measured with an NPA. The contour lines in a illustrate the density of beam neutrals. $\mathbf{c}$ Weight function of the simulated neutral fluxes in velocity space (pitch, energy), see Sect. 6 for more details. The figure is taken from reference Schneider et al. (2015)

resolution, since the pitch value of fast ions depends on the direction of the local magnetic field. At the plasma edge, for example, the angle between the line-ofsight and the magnetic field is typically different compared to the one in the plasma center. As an example, Fig. 11 shows a simulation of the birth location of detected neutrals together with the corresponding pitch values. The simulation shows a clear contribution from the plasma edge due to passive charge-exchange as well as an active contribution from the plasma center. The pitch values of the active and passive contributions differ (about 0.65 for the active contribution and about 0.5 for the passive one).

For a quantitative interpretation of NPA measurements, forward modelling is needed. Here, several codes exist such as FIDASIM (Heidbrink et al. 2011) or DOUBLE (Kislyakov et al. 2001). These codes need the background density of neutrals from the walls and a given fast-ion distribution function as inputs. The codes determine the probability for charge-exchange between a given donor neutral and the fast or thermal particle and then follow the neutralized particles through the plasma. The re-ionization process along the straight path through the plasma is also accounted for. Accounting for the aperture of the diagnostic and the size of the detector, NPA fluxes can be calculated in absolute units and the corresponding energies and pitch values can be analyzed.

In contrast to passive NPA measurements, active NPA measurements with a modulated NBI allow well localized measurements by subtracting the passive fluxes measured when the beam is off. This allows highly sensitive and well localized measurements at one given pitch value. Thus, one obtains information about fast ions at a specific pitch, $\mathrm{R}$ and $\mathrm{z}$ location with good resolution in energy. Different parts of the fast-ion phase space can only be addressed by installing several detectors as done at NSTX (Liu et al. 2016). Such multi-detector NPA systems are, however, not routinely employed because of the detector size and the limited access to most fusion devices. New developments, such as an in-vessel scintillator-based NPA, might provide a better coverage of the phase space in future experiments. 


\subsection{FIDA}

In contrast to the small velocity-space regions observed by NPA detectors, FIDA spectroscopy covers large parts of fast-ion-velocity space, or even complete if a few judiciously arranged detectors are available. However, it exhibits relatively poor resolution in velocity space and can, thus, be seen as a complementary diagnostic to NPAs. FIDA spectroscopy is based on the analysis of the Doppler-shifted Balmeralpha emission ( $n=3$ to $n=2$ at $656.1 \mathrm{~nm}$ ), emitted by the neutralized particles after charge exchange (see Fig. 12). As can be seen in the cross sections plotted in Fig. 9, the charge-exchange cross section from $n=1$ into the $n=3$ state is up to 20 times smaller than the corresponding cross section into the $n=1$ state. Thus, about $5 \%$ of the fast neutrals are in the $n=3$ state after charge-exchange reactions. This percentage is much larger than the typical equilibrium fraction of $n=3$ neutrals. Hence, an overpopulation of the $n=3$ state is present after the charge-exchange process, which provides strong, localized Balmer-alpha emission within the first few cms after neutralization.

Figure 13 illustrates that the population of the ground state $(n=1)$ of a deuterium neutral is typically three orders of magnitude larger than the population of the $n=3$ state, assuming typical plasma parameters, as given in Fig. 13. At $5 \mathrm{~cm}$, we considered a charge-exchange process with a $80 \mathrm{keV}$ ion and a relative collision energy of $30 \mathrm{keV}$. From that position on, we continue to plot the state distribution of the neutralized $80 \mathrm{keV}$ ion. Clearly, the $n=3$ state is overpopulated after charge exchange and then decays through the spontaneous emission of photons. As indicated, $69 \%$ of the FIDA emission is emitted within the first four centimeters after charge exchange, providing good spatial localization.

\subsection{Instrumentation}

The required diagnostic equipment to detect the Doppler-shifted Balmer-alpha light is basically the same as required for charge-exchange recombination spectroscopy (CXRS), used to infer ion temperature, rotation, and density measurements of impurity ions. Lenses collect radiation from the plasma in the visible range and fibers guide this light to a spectrometer that is typically located distant from the fusion device. The distance to the device avoids interference by the magnetic fields or neutrons that can have an effect on the camera attached to the spectrometer. Given that the spectrometer can reach $656 \mathrm{~nm}$ (the Balmer-alpha line) with a spectral range that is wide enough, every CXRS diagnostic can be used for FIDA measurements. However, for a dedicated diagnostic, several optimization criteria should be considered: the signal level of FIDA light is low compared with normal charge-exchange measurements. It is thus important to guarantee enough photons to be detectable by the camera, such that the FIDA signal level is well above the read-out noise level. Dedicated FIDA spectrometers are, therefore, operated using relatively wide entrance slits. This enhances the photon throughput, but also increases the instrumental broadening function, i.e., reduces the wavelength resolution. Moreover, measuring the whole D-alpha spectrum with good SNR is hardly possible with a 
Fig. 12 Sketch of the FIDA emission process. The figure is taken from reference Heidbrink et al. (2004)

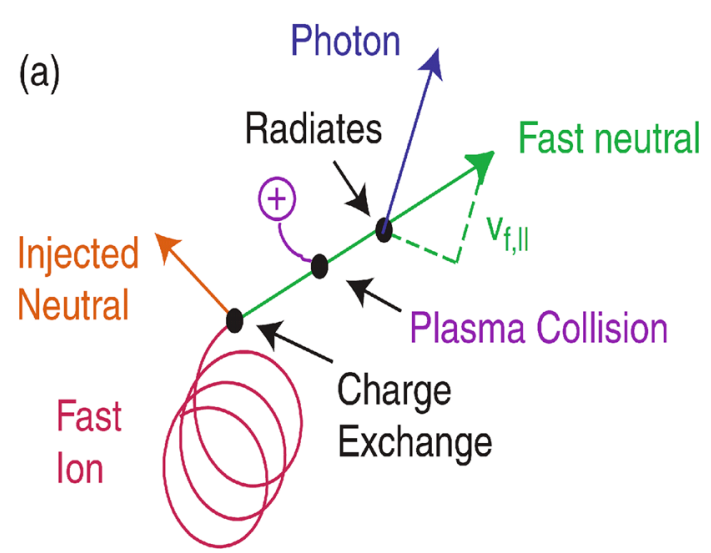

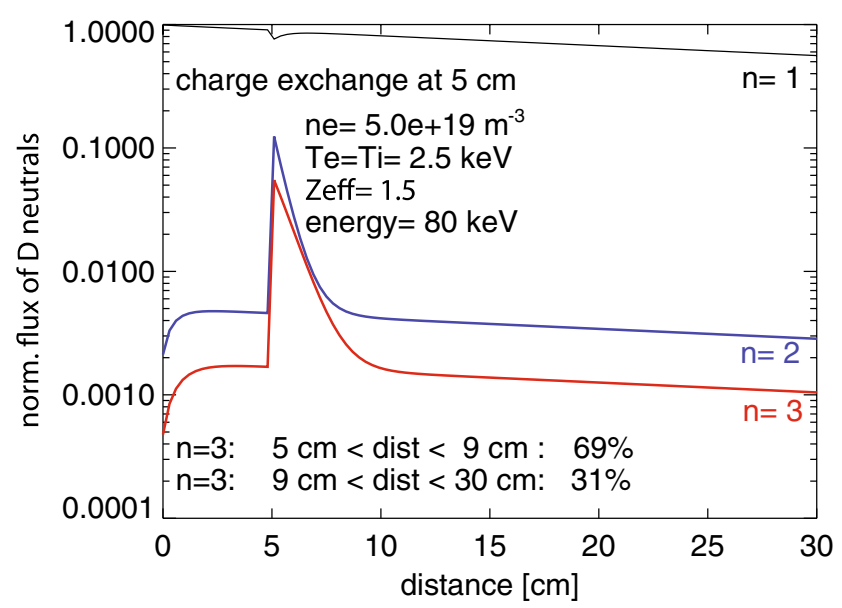

Fig. 13 Population of the quantum states of a deuterium neutral with $80 \mathrm{keV}$ that undergoes charge exchange after $5 \mathrm{~cm}$ with an $80 \mathrm{keV}$ ion with a relative collision energy of $30 \mathrm{keV}$. From that position on the population of states of the neutralized ion is displayed

standard CXRS spectrometer, because the edge-D-alpha emission is very bright and would cause saturation effects of the camera. There are studies of very low-density plasmas during which the unshifted Balmer-alpha light does not cause too strong saturation of the CCD [e.g., at TCV Geiger et al. (2017)], but typically standard CXRS diagnostics used for FIDA are limited to measure either blue or red-shifted FIDA light. As an example, first FIDA measurements at DIII-D (Heidbrink et al. 2004; Muscatello et al. 2010), ASDEX Upgrade (Geiger 2011), MAST (Michael et al. 2013), or LHD (Osakabe et al. 2008; Ito 2010; Murakami et al. 2008) keep the strong unshifted edge radiation away from the detector chip by focussing only on blue- or red-shifted radiation. Here, it should be noted that LHD used hydrogen as main-ion and fast-ion species. Instead of 'FIDA', the term 'Fast Ion Charge eXchange Spectroscopy' (FICXS) is used). Nowadays, dedicated FIDA systems at 
DIII-D, ASDEX Upgrade (Weiland et al. 2016), NSTX (Podestà 2008; Bortolon 2010), or EAST (Hou et al. 2016) are equipped with additional lenses and a wire in the intermediate image that blocks the edge-D-alpha radiation and permits simultaneous measurements of the complete Balmer-alpha spectrum (roughly between 645 $\mathrm{nm}$ and $665 \mathrm{~nm}$ ).

Figure 14 shows a photograph of such a dedicated FIDA instrument at NSTX. The first lens (2) situated after the entrance slit (1) parallelizes the light for the grating (3), and the second lens (4) focuses the dispersed radiation onto the image plane (5). Standard spectrometers would have the detector placed here. However, FIDA systems make use of a wire or a thin neutral density filter at this position to block the cold D-alpha emission. In addition, one or two additional lenses (6) are applied to project the intermediate image on the detector (7).

\subsection{Energetic ion measurements by FIDA}

A passive FIDA spectrum and an active FIDA spectrum, measured at ASDEX Upgrade, are potted in Fig. 15a, b on a semi-logarithmic scale. The radiation around $656 \mathrm{~nm}$ is blocked by a wire in the spectrometer setup.

The passive spectrum consists of bremsstrahlung and line radiation mainly emitted close to the plasma edge. As already mentioned, the Balmer-alpha radiation from the plasma edge is particularly intense and dominates the spectrum. In blue, we depict a synthetic edge Balmer-alpha line with a realistic intensity. The line is broadened by the Doppler effect (here, we assume $10 \mathrm{eV}$ ), and by Stark splitting and Stark broadening which appears in plasma regions, where the electron density is large (here, we consider an electron density of $10^{20} / \mathrm{m}^{3}$ ). In addition, impurity lines are present, of which carbon and oxygen lines have been identified in the plot. Finally, passive FIDA radiation can be observed which is present due to chargeexchange reactions between fast ions and neutrals from the walls. In Fig. 15a, simulated passive FIDA radiation from FIDASIM is plotted in red assuming a neutral density profile from TRANSP/FRANTIC. In particular, when the populations of fast ions and neutrals (strongly decaying towards the center) are both large, e.g., during off-axis NBI experiments, this type of FIDA radiation can be observed. Due to the missing spatial localization of passive FIDA light, the interpretation is difficult.

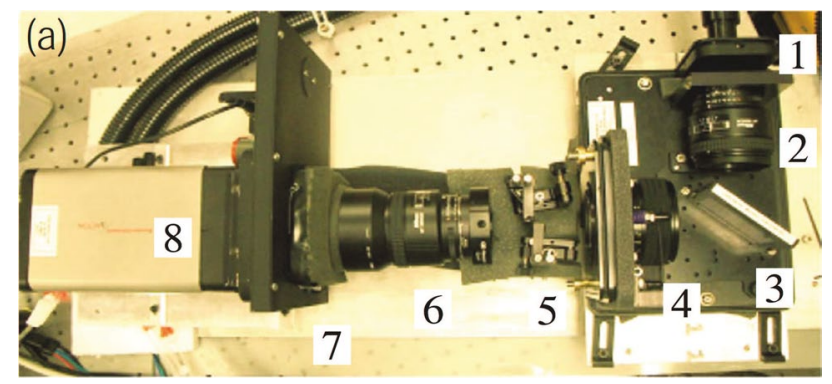

Fig. 14 FIDA spectrometer used at NSTX, representative for FIDA spectrometers with a filter to block edge-D-alpha radiation. The figure is taken from Podestà (2008) 

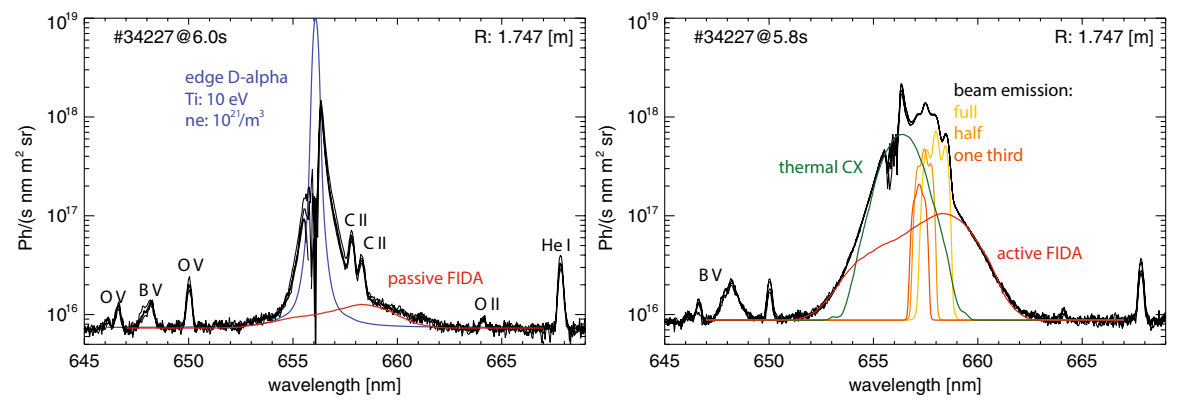

Fig. 15 Active and passive FIDA spectra measured at ASDEX Upgrade together with predictions from FIDASIM

Thus, passive FIDA is typically avoided or removed from the spectrum by studying the active signal using modulation of the neutral beam, whose path is crossed by the lines-of-sight.

A spectrum with additional active contributions is plotted in Fig. 15b, together with the simulation from FIDASIM. The simulation consists of charge-exchange radiation emitted by neutralized thermal ions (thermal cx, green) and by neutralized fast ions (red), as well as by impurity ions. In addition, the beam emission is present in active spectra, which is the direct radiation from fast neutrals injected by NBIs. The beam emission consists for positive NBI sources of the full, half, and one third energy components, which are each split by the motional Stark effect. When selecting lines-of-sight for FIDA spectroscopy, it is essential that the beam emission does not overlap with the FIDA emission. This can be achieved by viewing lines close to perpendicular to the NBI path, yielding relatively small Doppler shifts of the beam emission. The active FIDA radiation is localized along the NBIs. Using radially distributed lines-of-sight, radial profiles with information on fast ions can be observed. In particular, when using lines-of-sight that are tangential to the magnetic field lines at the intersection with a given NBI path, good spatial resolution can be obtained. To generate radial profiles, each spectrum needs to be integrated within a given wavelength range and the passive contribution needs to be subtracted. Here, either frames without NBI are subtracted or a flat line describing bremsstrahlung measured elsewhere in the spectrum (e.g., 664-667 nm) is subtracted. When selecting a certain wavelength range for the profiles (i.e., a certain Doppler shift), a welldefined part of the fast-ion-velocity space is addressed.

The Doppler shift of the FIDA radiation contains information on the fast-ionvelocity-space distribution. In general, a given Doppler shift can be related to a minimum energy that the ion must have to produce this Doppler shift. The larger the Doppler shift, the larger this minimum energy is. However, an upper limit for the ion energy for a given Doppler shift cannot be given. Low Doppler shifts can be produced due to highly energetic ions if these move close to perpendicular to the viewing geometry. This can be seen by the nature of the Doppler effect:

$$
\lambda_{\mathrm{D}}-\lambda_{0}=u \lambda_{0} / c \text {. }
$$


Here, $c$ is the speed of light in and $\lambda_{0}$ is the unshifted D-alpha wavelength $(656.1$ $\mathrm{nm}$ ), and $u$ is the projected velocity of the ion onto the line-of-sight.

To describe the observed fast-ion-velocity-space region when analyzing a given Doppler shift with a given viewing geometry, weight functions are used (Heidbrink 2010; Salewski et al. 2014a), see Sect. 6. The weight functions can, e.g., be calculated by FIDASIM and yield the expected FIDA photon flux when first multiplying a given weight function with a fast-ion distribution function and after integration in fast-ion-velocity space.

If all Doppler shifts were accessible, information on the whole fast-ion-velocity-space distribution would be obtained from a single line-of-sight (Salewski et al. 2012). However, in particular, at low wavelength shifts, the FIDA radiation is superimposed by the beam emission, thermal charge-exchange emission, and the cold edge-D-alpha line. Thus, a given viewing geometry can only probe a certain part of the fast-ion-velocity distribution. Different viewing geometries are needed to obtain a full coverage. An example is the five-view FIDA system at ASDEX Upgrade (Weiland et al. 2016), as plotted in Fig. 16. This FIDA system with five different viewing directions was designed to allow studies of the fast-ion-velocity distribution function by velocity-space tomography. The velocity-space coverage of this five-view FIDA system is illustrated in Weiland et al. (2016). Examples of measured fast-ion-velocity distribution function are presented in Sect. 6.

For the quantitative analysis, the measured profiles can be compared with radial profiles from FIDASIM. Here, it should be noted that an absolute calibration of CXRS diagnostics is demanding. Cameras might degrade with time or vacuum windows might get coated with layers, such that an initially good calibration might not be trustworthy after some time. In the case of FIDA spectroscopy, it is, therefore, recommended to cross check the intensity calibration by additionally analyzing the beam emission and the level of bremsstrahlung. Both additional spectral components are simulated by FIDASIM and can be compared with the measurement. Only if this check is successful, reliable conclusions based on

Fig. 16 Geometrical arrangement of the line-of-sight arrays at ASDEX Upgrade (Weiland et al. 2016)

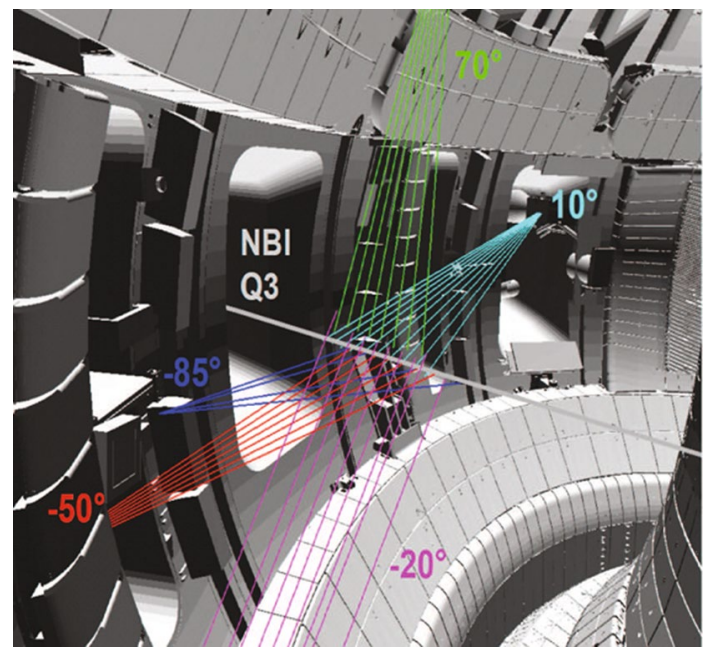


the FIDA data can be made. Another approach is to analyze the ratio of the FIDA signal to the beam emission intensity (BES). Since FIDASIM simulates both contributions, the comparison of the measured FIDA/BES signal with the simulated one is independent of the intensity calibration. An example of a measured FIDA/ BES signal from ASDEX Upgrade is plotted in Fig. 17.

The analysis of fast ions with energies well above $100 \mathrm{keV}$ is difficult. The most important charge-exchange cross section for FIDA is the one from $n=1$ to $n=3$, because it directly populates the atomic state responsible for the Balmeralpha emission. This cross section peaks at the relative collision energy of about $30 \mathrm{keV} / \mathrm{amu}$. At higher collision energies, such as $200 \mathrm{keV} / \mathrm{amu}$, the cross section is lower by more than two orders of magnitude. In addition, the FIDA emission from highly energetic ions can spread over a large wavelength range due to the gyro-motion and the Doppler effect. Thus, the expected FIDA intensity per wavelength bin reduces significantly with increasing energy (Fig. 18).

At low signal levels, the measurement becomes affected by the background noise. This background noise consists of hardware-related noise such as the camera readout noise. In addition, it consists of photon noise, which follows the Poisson statistic $\left(\sigma=\sqrt{N_{\text {photons }}}\right)$. While read-out noise can be almost eliminated by long exposure times or the application of an electromagnetic (EM) gain in modern CCD cameras, the level of photon noise is difficult to reduce. In particular, the presence of bremsstrahlung limits the analysis of FIDA radiation, because this radiation is present in FIDA spectra as a flat offset.

In cases, where the square root of the number of photons from bremsstrahlung exceeds the number of photons from the FIDA process, the noise level is larger than the actual measurement. Thus, FIDA spectroscopy is not possible, since it is not possible to discriminate between noise and FIDA radiation. This is typically the case in plasmas with high-electron densities, where the level of bremsstrahlung is high and where the NBI attenuation is strong, reducing the number of donor neutrals.

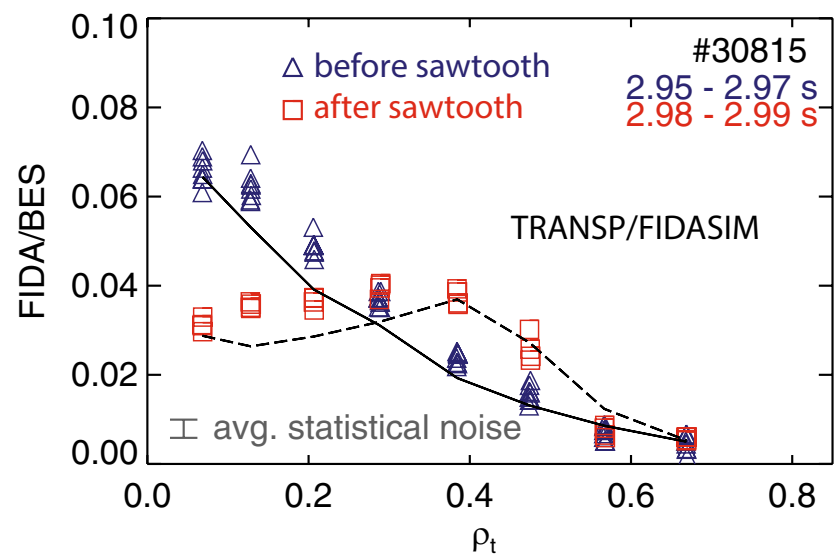

Fig. 17 Radial FIDA/BES profiles measured before and after a sawtooth crash in ASDEX Upgrade. The measurement is compared with synthetic profiles from FIDASIM, based on predicted fast-ion distribution functions from TRANSP (Geiger et al. 2015a) 

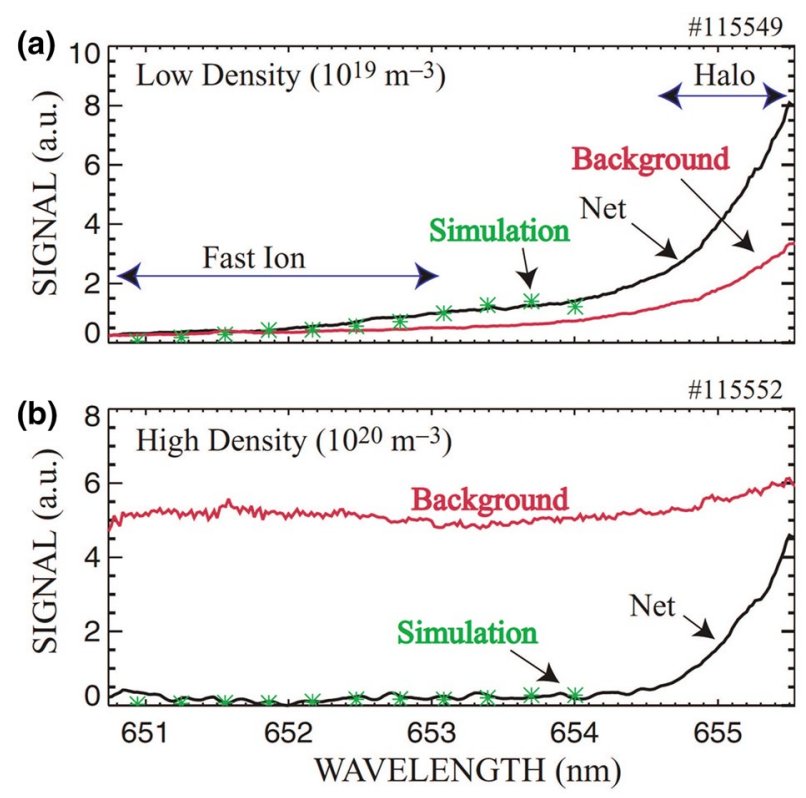

Fig. 18 FIDA spectra measured at DIII-D for two different plasma densities. The figure is taken from Heidbrink et al. (2004)

By increasing the photon throughput of the spectrometer or using longer exposure times, the situation can be improved a bit, since photon noise scales with $\sqrt{N_{\text {photons }}}$. A clear condition, up to which density FIDA measurements are possible, cannot be provided, because this additionally depends on the fast-ion and impurity concentrations and NBI power and energy. With respect to ITER, one can, however, already state that core localized FIDA measurements will be very challenging. High-electron densities, low fast D-ion densities, and low donor densities due to high-energy NBIs (the higher the energy, the less particles are injected at a given power) will result in weak FIDA signal strengths. In combination with the expected high levels of bremsstrahlung, the FIDA signal is likely going to be obscured by photon noise.

\section{Collective Thomson scattering}

The collective Thomson scattering (CTS) diagnostic is not as widespread as FIDA, NES, or GRS. However, it plays an increasingly important role in fast-ion research, since it is foreseen for as $\alpha$-particle diagnostic at ITER (Salewski et al. 2009; Bindslev et al. 2004; Tsakadze et al. 2008; Korsholm et al. 2016; Leipold et al. 2009). It can often be installed comparatively easily in devices with ECRH systems. CTS is an active, non-perturbative diagnostic with good temporal and spatial resolution. CTS diagnostic based on a far-infrared laser for ion temperature measurements was initially used at the TCA tokamak (Behn et al. 1989). Later, the CTS diagnostics at 
TFTR (Woskoboinikow et al. 1983; Machuzak et al. 1995), JET (Bindslev 1999a, b), TEXTOR (Bindslev et al. 2006; Michelsen 2004), ASDEX Upgrade (Meo et al. 2010, 2008, Salewski et al. 2010), LHD (Nishiura et al. 2014; 2010; Kubo et al. 2010), W7-AS (Suvorov et al. 1995), and FTU (Orsitto et al. 1999) used microwaves as a source of probing radiation for the fast-ion CTS. The diagnostic is also used for measurements of the ion temperature (Stejner et al. 2013, 2015), isotope ratio (Korsholm et al. 2011; Stejner et al. 2011, 2013, 2012a, b), and plasma rotation (Stejner et al. 2015, 2016). In inertial confinement fusion experiments, laser-based CTS is regularly used for ion temperature measurements (Glenzer et al. 1997; Ross et al. 2010). The infrared-laser-based diagnostic has been used in the JT-60U tokamak (Kondoh et al. 2003; Kondoh 1997; Kondoh et al. 2007; Kondoh 2007) and was considered as an option for ITER (Kondoh 2007; Bindslev et al. 2004), but in the end, the microwave-based diagnostic was preferred. Here, we concentrate on microwave-based design of the diagnostic.

\subsection{Physics principles}

The injected electromagnetic radiation accelerates the charged particles in the plasma which in turn radiate which is referred to as scattering. As the ions are much more massive than electrons, scattering off electrons dominates scattering off ions by orders of magnitude. The scattering off electrons bears signatures of microscopic fluctuations in the plasma. Of particular interest for fast-ion CTS are the fluctuations induced by fast ions. The acceptance cone of the antenna, which is often called the receiver beam, and the probe beam intersect in the so-called overlap volume which defines the measurement location in the plasma. Figure 19 depicts the geometry of CTS: $\mathbf{k}^{\mathbf{i}}$ is the wave vector of incident (probing) radiation, $\mathbf{k}^{\mathbf{s}}$ is the wave vector of the received scattered radiation, and $\mathbf{k}^{\mathbf{\delta}}$ defines the direction along which the fluctuations are resolved via the momentum matching condition:

$$
\mathbf{k}^{\mathbf{\delta}}=\mathbf{k}^{\mathbf{s}}-\mathbf{k}^{\mathbf{i}}
$$

If the Salpeter parameter $\alpha=1 /\left(k^{\delta} \lambda_{D}\right)>1$ Salpeter (1960), the scattered radiation bears a signature of collective fluctuations in the plasma, i.e., those induced by ion motion and MHD activity. Here, $k^{\delta}$ is the magnitude of $\mathbf{k}^{\boldsymbol{\delta}}$ and $\lambda_{D}$ is the Debye length. An angular frequency shift in the scattering radiation:

$$
\omega^{\delta}=\omega^{\mathrm{s}}-\omega^{\mathrm{i}},
$$

which corresponds to the energy matching condition, can be approximately related to an ion velocity, $\mathbf{v}_{\text {ion }}$, by

$$
\omega^{\delta} \approx \mathbf{v}_{\text {ion }} \cdot \mathbf{k}^{\mathbf{\delta}}=u k^{\delta} .
$$

Here, $\omega^{\mathrm{s}}$ and $\omega^{\mathrm{i}}$ are the angular frequencies of the scattered and incident radiation and $u$ is the projection of the ion velocity onto the direction of $\mathbf{k}^{\mathbf{\delta}}$.

The spectral power density of received scattering radiation is described by the equation of transfer 


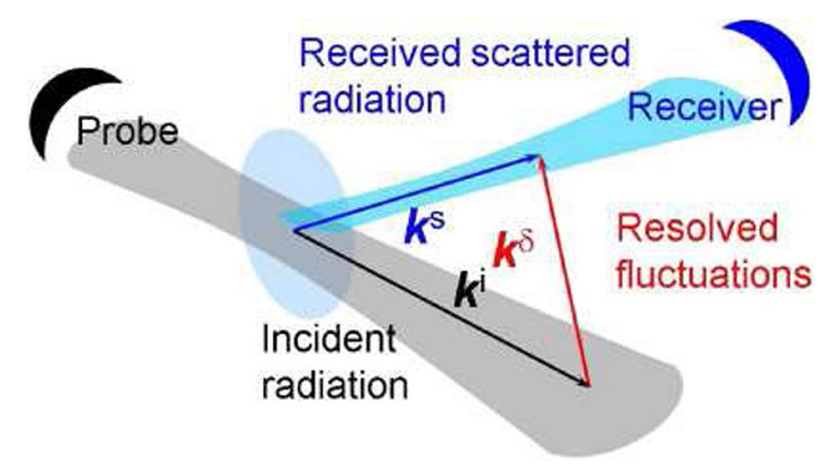

Fig. 19 Geometry of CTS: $\mathbf{k}^{\mathbf{i}}$ is the wave vector of incident (probing) radiation, $\mathbf{k}^{\mathbf{s}}$ is the wave vector of the received scattering radiation, and $\mathbf{k}^{\mathbf{\delta}}$ is the wave vector of resolved fluctuations. The figure is taken from Moseev et al. (2011)

$$
\frac{\partial P^{\mathrm{s}}}{\partial \nu}=P^{\mathrm{i}} O_{\mathrm{b}} \omega^{\mathrm{i}} \omega^{s} r_{\mathrm{e}}^{2} n_{\mathrm{e}} \frac{G S\left(k^{\delta}, \omega^{\delta}\right)}{2 \pi c^{2}},
$$

where $P^{\mathrm{i}}$ is the probing power; $O_{\mathrm{b}}$ is the so-called overlap integral which represents a measure of intersection between the probe beam and the receiver beam (Bindslev 1996); $\omega^{\mathrm{i}}$ and $\omega^{\mathrm{s}}$ are the angular frequencies of the incident and scattering waves; $r_{\mathrm{e}}$ is the classical electron radius; $n_{\mathrm{e}}$ is the electron density; $c$ is the speed of light in vacuum; $G$ is the so-called geometrical form factor which quantifies the efficiency of scattering from the probe beam to the receiver beam (Hughes et al. 1989); and $S$ is the so-called scattering function which defines the shape of the scattering spectrum as a function of plasma parameters, in particular the projection of the fast-ionvelocity distribution function. The scattering function $S\left(k^{\delta}, \omega^{\delta}\right)$ is derived in several approximations: electrostatic (Hughes et al. 1989) and fully electromagnetic (Bindslev 1996). The scattering function depends on the projection of the fast-ion-velocity distribution function onto the $\mathbf{k}^{\boldsymbol{\delta}}$ direction:

$$
g(u)=\iiint_{R^{3}} f(\mathbf{v}) \delta\left(u-\frac{\mathbf{k}^{\delta} \cdot \mathbf{v}}{k^{\delta}}\right) \mathrm{d} v_{\|} \mathrm{d} v_{\perp}^{1} \mathrm{~d} v_{\perp}^{2} .
$$

For NBI and ICRF scenarios, this 1D projected fast-ion-velocity distribution function depends strongly on the angle, $\phi$, between $\mathbf{k}^{\delta}$ and the local magnetic field vector in analogy to FIDA.

\subsection{Instrumentation}

The CTS diagnostic consists of two main systems: one system provides the probing radiation and the other system detects the scattered radiation. Typically, gyrotrons are used as sources of the probing radiation: using microwaves allows much 
flexibility in the choice of the geometry to ensure a Salpeter parameter greater than one. For shorter wavelengths, only forward scattering geometries ensure the collective scattering regime. Infrared lasers with a wavelength of $10.6 \mu \mathrm{m}$ have also been used as sources of probe radiation, but the scattering angle between the incident and scattered radiation had to be very small, less than $1^{\circ}$, to satisfy the Salpeter criterion. CTS diagnostics based on infrared lasers was used on the ATF torsatron (Richards et al. 1993) and the JT-60U tokamak (Lee and Kondoh 2000; Kondoh et al. 2003; Kondoh 2007). A feasibility study of using infrared-laser-based CTS for ITER was done (Bindslev et al. 2004), but it was found that $60 \mathrm{GHz}$ microwave CTS has the best performance.

Microwave-based CTS for fast-ion studies has been successfully operated on TEXTOR (Bindslev et al. 2006; Moseev et al. 2011; Nielsen et al. 2011, 2008, 2010; Bindslev et al. 2007; Korsholm et al. 2010), ASDEX Upgrade (Salewski et al. 2010; Nielsen et al. 2015; Meo et al. 2010, 2008; Rasmussen et al. 2015, 2016; Jaulmes et al. 2016), LHD (Nishiura et al. 2014, 2010; Kubo et al. 2010), Wendelstein-7AS (Suvorov et al. 1995; Shalashov et al. 2003), and FTU (Orsitto et al. 1999). TEXTOR and Wendelstein 7-AS are now out-of-service.

In CTS diagnostics, the probing radiation and the scattered radiation pass through transmission lines. The transmission lines often include steerable mirrors providing geometrical flexibility, polarizers [in some implementations of the diagnostics polarizers are incorporated into the receiver (Leipold et al. 2016)], and a matching optics unit which is a coupling device between the transmission line and the source of probing radiation or the microwave receiver. The transmission line can be quasioptical, as at TEXTOR (Nielsen et al. 2008) or Wendelstein 7-X (Erckmann 2007), or made of oversized waveguides as on ASDEX Upgrade (Furtula et al. 2012), LHD (Shimozuma 2010), Wendelstein 7-AS (Suvorov et al. 1995), FTU (Orsitto et al. 1999), and is being designed for ITER (Korsholm et al. 2016). Often, CTS shares the transmission lines with the ECRH system, as at ASDEX Upgrade, LHD and Wendelstein 7-AS, which means that two transmission lines are not available for ECRH during CTS measurements. ITER will have dedicated microwave transmission lines for the CTS diagnostic.

CTS receivers are highly sensitive, heterodyne radiometers. A schematic of the receiver at ASDEX Upgrade is shown in Fig. 20, left panel.

The CTS diagnostic does not only receive the scattered radiation but any other microwave radiation in the detection frequency range. These are ideally limited to electron cyclotron emission (ECE) and stray radiation from the gyrotron. The scattering power is typically 11-12 orders of magnitude smaller than the probe power and is typically in the $\mu \mathrm{W}$ range. The exact quantity is determined by the beam overlap integral, the electron density, the scattering function, and the geometrical form factor (Bindslev 1992). The probe power is usually in the range of several hundred $\mathrm{kW}$. $1 \mathrm{MW}$ is foreseen for CTS at ITER. If even a tiny fraction of the injected radiation entered the receiver as stray radiation, several components in the receiver would be damaged. Therefore, the receiver must be protected by a notch filter (or a cascade of notch filters). The depth of the notch is around - $100 \mathrm{~dB}$ (Furtula et al. 2010) in the stopband of 200-300 MHz width. The insertion loss is about $-2 \mathrm{~dB}$ per notch filter. In addition to the notch filters, the radio-frequency (RF) part of the receiver is 


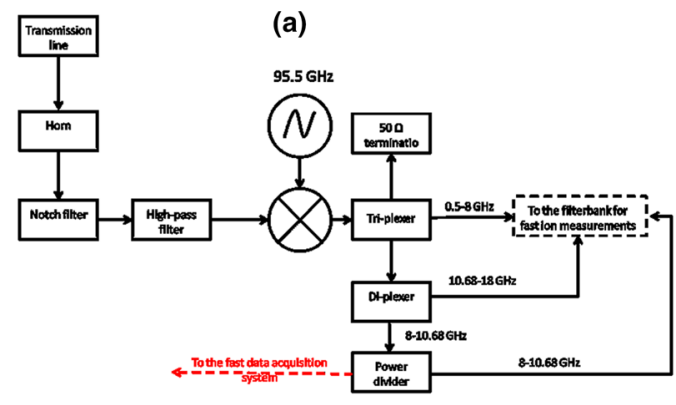

(b)

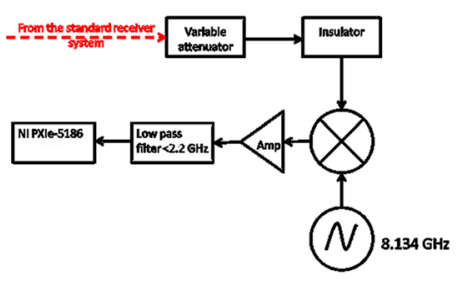

Fig. 20 Schematic of the CTS receiver at ASDEX Upgrade. The left panel a depicts all parts of the receiver from the horn to the filter bank and an additional mixing stage with the fast data acquisition is shown on the right panel (b). More detailed description is available in reference Stejner et al. (2014)

protected by a variable voltage-controlled attenuator (VCVA) with maximum attenuation of $-40 \mathrm{~dB}$. The VCVA is needed to protect the receiver when the probe is switched on or off. At these times, the gyrotron frequency chirps and may potentially drift outside the stopband of the notch filter, which may cause receiver damage.

Another necessary component of the CTS receiver is a band-pass filter. The bandpass filter ensures that the RF signal in the desired frequency range only is transmitted further for down-mixing, thus avoiding aliasing effects. Some CTS receivers, for example, the CTS receiver at ASDEX Upgrade, are additionally equipped with a low-pass filter to protect the receiver from high-frequency stray radiation from the ECRH gyrotrons (Furtula et al. 2012). Fast-ion CTS at ASDEX Upgrade operates at $105 \mathrm{GHz}$, where the gyrotrons for ECRH often operate at $140 \mathrm{GHz}$. An insulator before the mixer is needed for suppression of backreflections from the mixer.

The RF signal is typically mixed down, so that the resulting frequency of the signal is in the range of 10-20 GHz. This frequency range is comfortable to work with as there is a large variety of affordable low-noise microwave components. After amplification, the intermediate frequency signal comes to a triplexer as on TEXTOR (Stejner et al. 2010) or a four-way power splitter (a combination of a triplexer with a diplexer) as on ASDEX Upgrade (Stejner et al. 2014). At ASDEX Upgrade, only three outputs are used and the fourth is used only for auxiliary purposes. The power splitting is done as the amplitude of the spectral power density of the scattered radiation strongly depends on the frequency, such that it is advantageous to use different amplifications for various frequency ranges. Another reason is to avoid the non-linear effects of the amplifiers on the spectrum when not only the signal is amplified but its harmonics as well. In the vicinity of the probing frequency, the scattering spectrum is dominated by the contributions from thermal particles and has large spectral power density, often comparable with the spectral power density of the ECE background. This part of the spectrum needs the least amount of amplification. The wings of the frequency spectrum of the scattering radiation are dominated by energetic particles. The spectral power density of the scattering signal is typically two orders of magnitude lower and thus the signal requires stronger amplification. After amplification, the signal is fed to a filter bank which has 30-50 
channels. As an example, the frequency response of intermediate frequency filters in the filter bank of the $77 \mathrm{GHz}$ CTS diagnostic on LHD is displayed in Fig. 21. For the frequency channels near probing frequency, the band-pass filters have $3 \mathrm{~dB}$ width of $100 \mathrm{MHz}$. The frequency channels in the filterbank monitoring frequencies with large frequency shifts are usually designed to have larger width, because the signal in these fast-ion channels is much lower.

At the moment, fast acquisition systems with a bandwidth of several $\mathrm{GHz}$ and corresponding sampling rate are becoming a common in CTS (Stejner et al. 2010, 2014; Nishiura et al. 2014, 2010). For the use of fast acquisition cards, a fraction of the intermediate frequency signal is taken from one of the outputs of a power divider or triplexer, amplified, and mixed down once again to frequencies of 1-10 $\mathrm{GHz}$, where it can be sampled by the fast analog-to-digital converter (ADC). To obtain the frequency spectrum, the sampled signal is Fourier transformed. An example of the receiver which features the fast acquisition system is shown in Fig. 20. A key feature of receivers with the fast data acquisition is a very high-frequency resolution which is defined by the length of the Fourier window. A frequency resolution of about $1 \mathrm{MHz}$ is easily achievable. This allows the detection of spikes in the spectra that are not easily seen using filterbank-based detection. Filterbank-based detection systems integrate the signal over a much larger bandwidth on the order of $100 \mathrm{MHz}$. Therefore, narrow high-amplitude spikes as those shown in Fig. 22 lead to a small elevation of the signal which may be hard to distinguish from the fast-ion signature in the spectrum. The fast ADC in CTS receivers is typically used for the bulk-ion measurements which require high-frequency resolution. Such systems also allow measurements of the gyrotron frequency despite the depth of the notch filters, so that any drift in the gyrotron frequency can be tracked and accounted for in the

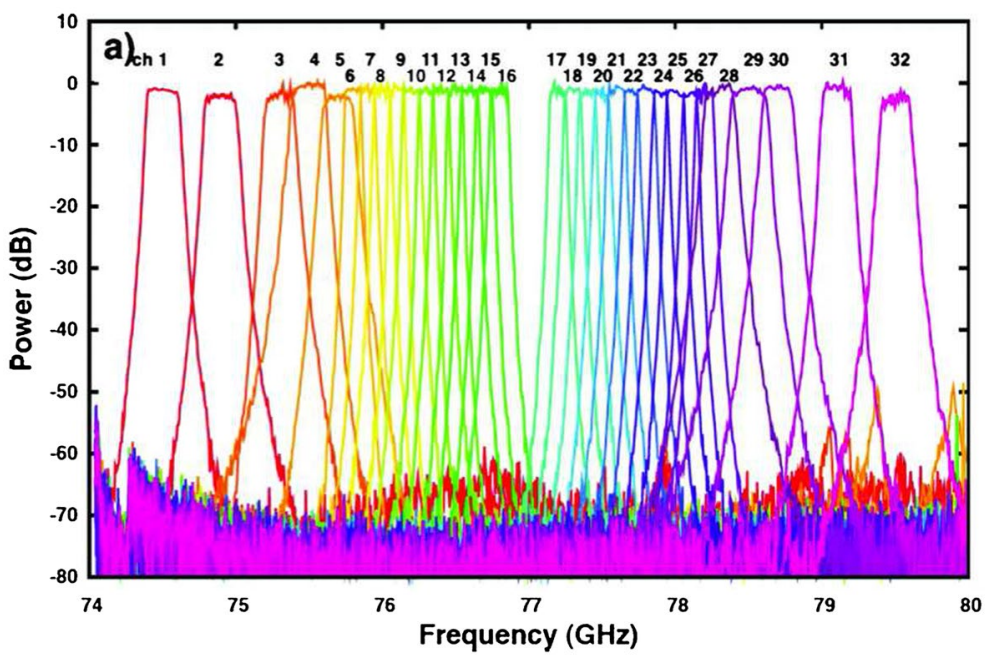

Fig. 21 Characteristics of band-pass filters in the filter bank of the CTS diagnostic at LHD. The figure is taken from Kubo et al. (2010) 


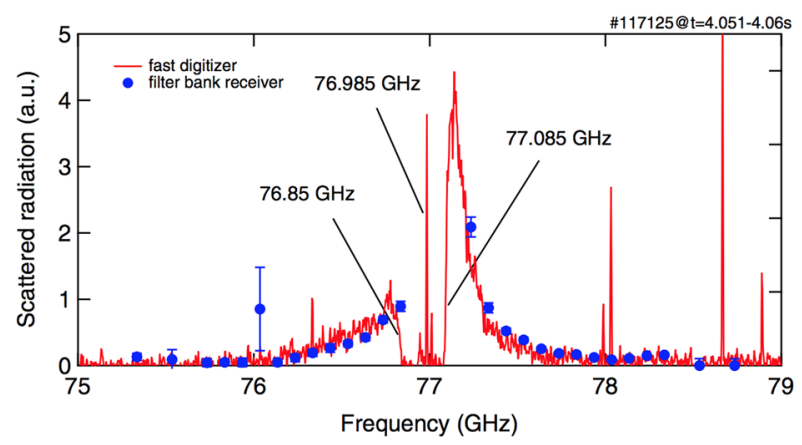

Fig. 22 Comparison of the CTS spectra obtained by the filterbank and the fast ADC at the LHD stellarator. The figure is taken from Nishiura et al. (2014)

data analysis. High-resolution CTS measurements could also be used for fast-ion measurements, but this has not yet been done.

\subsection{Operational regimes}

CTS is a flexible instrument, and this operational flexibility can be exploited to improve the SNR of the measurements. In the following section, we discuss the main control parameters of the diagnostic.

Beam overlap It is assumed in the CTS model that the received scattering radiation originates from the region, where the probe and receivers beams overlap. Quantitatively, it is described in the equation of transfer (Eq. (8)). The location of the overlap volume and the beam overlap $O_{b}$ are calculated using beam tracing or ray tracing codes such as TRAVIS (Marushchenko et al. 2014). However, the electron temperature and density profiles are not known precisely and microwaves can be strongly refracted. If the beam width is narrow or the location of the overlap volume is far from the launching and receiver mirrors, the location of the beam overlap volume may significantly differ from theoretical estimates. A scenario with a long distance between the measurement volume and the receiver mirrors and enhanced refraction occurs typically in measurements of ions with pitches close to \pm 1 . In such scenarios, the calculated mirror positions for the best possible overlap at a given location may serve only as an initial guess. The real mirror settings for the maximum overlap $O_{b}$ from the equation of transfer (8) are found during the so-called overlap sweep. During the sweep, the probe beam is fixed and the receiver beam is scanned across the probe beam. The scan is performed around the position of the theoretically defined overlap. The procedure is described in detail in references Nielsen et al. (2008); Moseev et al. (2011) and illustrated in Fig. 23. The sweep serves the purpose of finding the best beam overlap, and methodologically, it also establishes that the measurements are local.

Background subtraction The CTS receiver accepts microwaves in the predefined frequency range and polarization. Therefore, the scattered radiation is measured together with any other radiation referred to as background radiation. A prime 

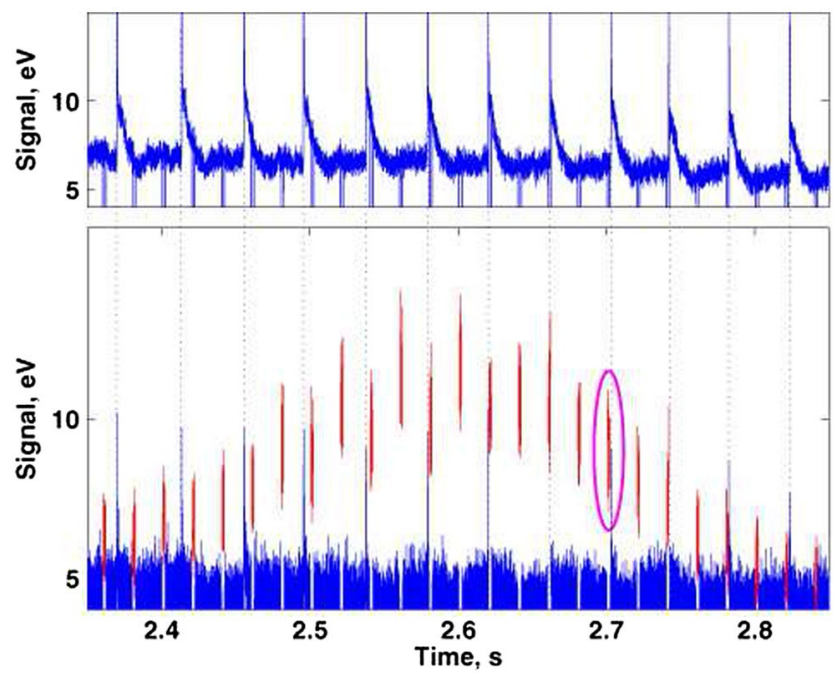

Fig. 23 Source modulation in the CTS experiment at TEXTOR during the so-called overlap sweep in shot 111,506 when the receiver beam is swept across the probing beam. The upper and lower panels depict the time traces of channels 42 and 37, respectively. Channel 42 receives no scattering radiation, only the ECE background. Therefore, neither the overlap sweep nor the power modulation of the probe are visible. Channel 37 receives the fast-ion scattering signal, and when the overlap between the receiver and probing beams is significant, the scattering signal emerges as the difference between the received power in the gyrotron-on (red) and gyrotron-off (blue) phases (Moseev et al. 2011)

source of the background for the microwave-based CTS is ECE. For the infraredlaser CTS, the sources of the background are thermal radiation of the first-wall surface and atomic emission lines. To subtract the background, the power of the probe beam is modulated. Then, the measured spectral power density of the scattering signal $\frac{\partial P^{s}}{\partial v}$ is

$$
\frac{\partial P^{s}}{\partial v}=\left.\frac{\partial P}{\partial v}\right|_{\mathrm{on}}-\left.\frac{\partial P}{\partial v}\right|_{\mathrm{off}},
$$

where $\left.\frac{\partial P}{\partial v}\right|_{\text {on }}$ and $\left.\frac{\partial P}{\partial v}\right|_{\text {off }}$ are the measured spectral power densities with the gyrotron switched on or off, respectively. Measured spectral power densities for a modulated gyrotron are displayed in Fig. 23. The time resolution is limited to about $4 \mathrm{~ms}$ with this technique, corresponding to $2 \mathrm{~ms}$ on and $2 \mathrm{~ms}$ off, as this is currently close to the modulation frequency limit for gyrotrons. However, the gyrotron power can be modulated about ten times faster, for example, between 90 and $10 \%$ of the full power. The better time resolution is sometimes highly advantageous if the signal varies on the millisecond time scale. With this technique, the background radiation cannot be subtracted, since there is still a small scattering component, but the difference in signal levels still allows the inference of the parameters of interest. One may also occasionally switch the gyrotron off in this scheme to get a measurement of the background. 
The spectral power density of the ECE background strongly influences SNR of the diagnostic (Bindslev 1992):

$$
\mathrm{SNR}=\frac{\partial P^{s}}{\partial \nu} \sqrt{\frac{\mathrm{WT}}{2\left(\frac{\partial P^{s}}{\partial \nu}+\left.\frac{\partial P}{\partial \nu}\right|_{\text {off }}\right)^{2}+2\left(\left.\frac{\partial P^{b}}{\partial \nu}\right|_{\text {off }}\right)^{2}}},
$$

where $W$ is the bandwidth of the frequency channel, $T$ is the integration time, and $\left.\frac{\partial P^{b}}{\partial v}\right|_{\text {off }}$ is the spectral power density of the background signal.

The amount of the background radiation strongly depends on the presence or absence of the ECE resonances in the plasma at the receiving frequencies. It also depends on the polarization of the receiver and on the plasma parameters. In fastion CTS, the frequency is chosen in a way that the waves do not meet a cutoff or an absorption/emission layer at positions with significant electron densities before the measurement volume. A sketch illustrating the dependence of the amount of ECE seen by a receiver as a function of the probing frequency is shown in Fig. 24. At the moment, a $300 \mathrm{GHz}$ gyrotron is under development for CTS at LHD, which would allow operation at the frequencies above the third harmonic of ECE with very small spectral power density of the background radiation (Yamaguchi et al. 2015).

The spectral power density of the scattering signal depends on the input power and on the scattering channel, $\mathrm{X}$ to $\mathrm{X}, \mathrm{O}$ to $\mathrm{O}, \mathrm{X}$ to $\mathrm{O}$, and $\mathrm{O}$ to $\mathrm{X}$, where $\mathrm{X}$ and $\mathrm{O}$ refer to the extraordinary and the ordinary modes, respectively. This dependency is described in the geometrical form factor in equation (8). The integration time should be chosen as compromise between long times for good SNR and short times, so that plasma parameters such as temperature, density, and fast-ion content do not change significantly during the averaging period.

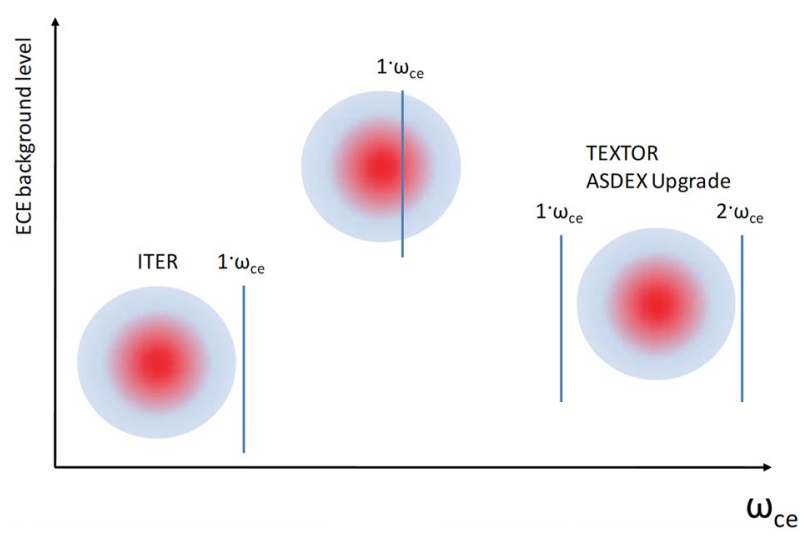

Fig. 24 The sketch illustrates the amount of the ECE background seen by the receiver from the position of ECE resonances in plasma at the detected frequencies. The cases of the fast-ion CTS at ASDEX Upgrade, TEXTOR, and future CTS at ITER are shown (Moseev 2011). Sometimes, resonances in the plasma after the measurement volume can be advantageous as they absorb part of the microwaves 


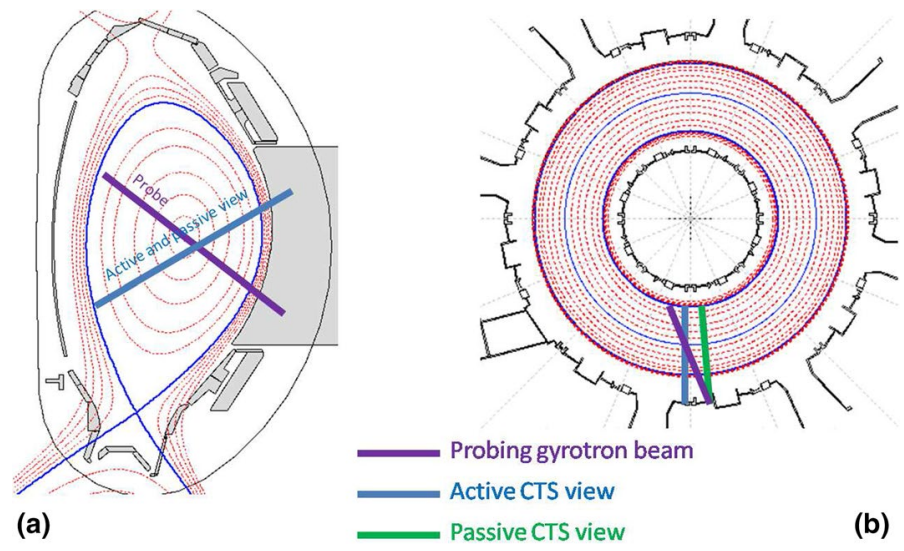

Fig. 25 Double receiver CTS setup at ASDEX Upgrade that allows the subtraction of overlap-independent spurious signal in the received scattering spectra. The passive CTS view does not intersect the probing beam. On the left panel (a), the poloidal cross section of ASDEX Upgrade is shown together with the probing beam (magenta), active and passive CTS receiver views (blue). The active and passive views have the same projection in this plane. On the right panel (b), the toroidal cross section of the setup is shown. The active and passive CTS receiver views are shown in blue and green, respectively

Sometimes, the spectral power density in the gyrotron-on period is larger than that in the gyrotron-off period even if the probe and receiver beams do not overlap. Such kind of spurious signal also occurs when the probe and receiver beams do overlap and are then hard to distinguish from scattering radiation. Such spurious signal can sometimes be subtracted using a second receiver not intersecting the probe beam which is referred to as a passive view. A second receiver for the background subtraction is currently installed on ASDEX Upgrade (Nielsen et al. 2015) and is planned for the CTS diagnostic at LHD. After background subtraction, quantitative agreement between the measured and simulated fast-ion-velocity distribution functions in the discharges with the CTS spectra that are contaminated by the spurious signal is achieved. An installation of the double receiver CTS diagnostic is shown in Fig. 25. Ideally, the active and the passive views should measure the same background when the gyrotron is off. This technique works best for receiver beams that are perpendicular to the magnetic field, such that refraction is small and the two beams are toroidally displaced but otherwise similar. Sometimes, spurious signal can also be mitigated by the fast modulation technique described above.

\subsection{Energetic ion measurements by CTS}

The diagnostic is capable of resolving fast-ion dynamics on the time scales which are limited by the SNR or by the gyrotron modulation frequency. Fast-ion redistribution due to the sawtooth oscillations has been measured by CTS (Nielsen et al. 2010). Qualitative (Nielsen et al. 2008; Salewski et al. 2010) and quantitative agreement (Nielsen et al. 2015; Moseev et al. 2011; Rasmussen et al. 2015) between measurements and Monte Carlo simulations in MHD quiescent scenarios has been achieved. Figure 26 demonstrates quantitative [panels (a) and (b)) and qualitative (panels (c) 

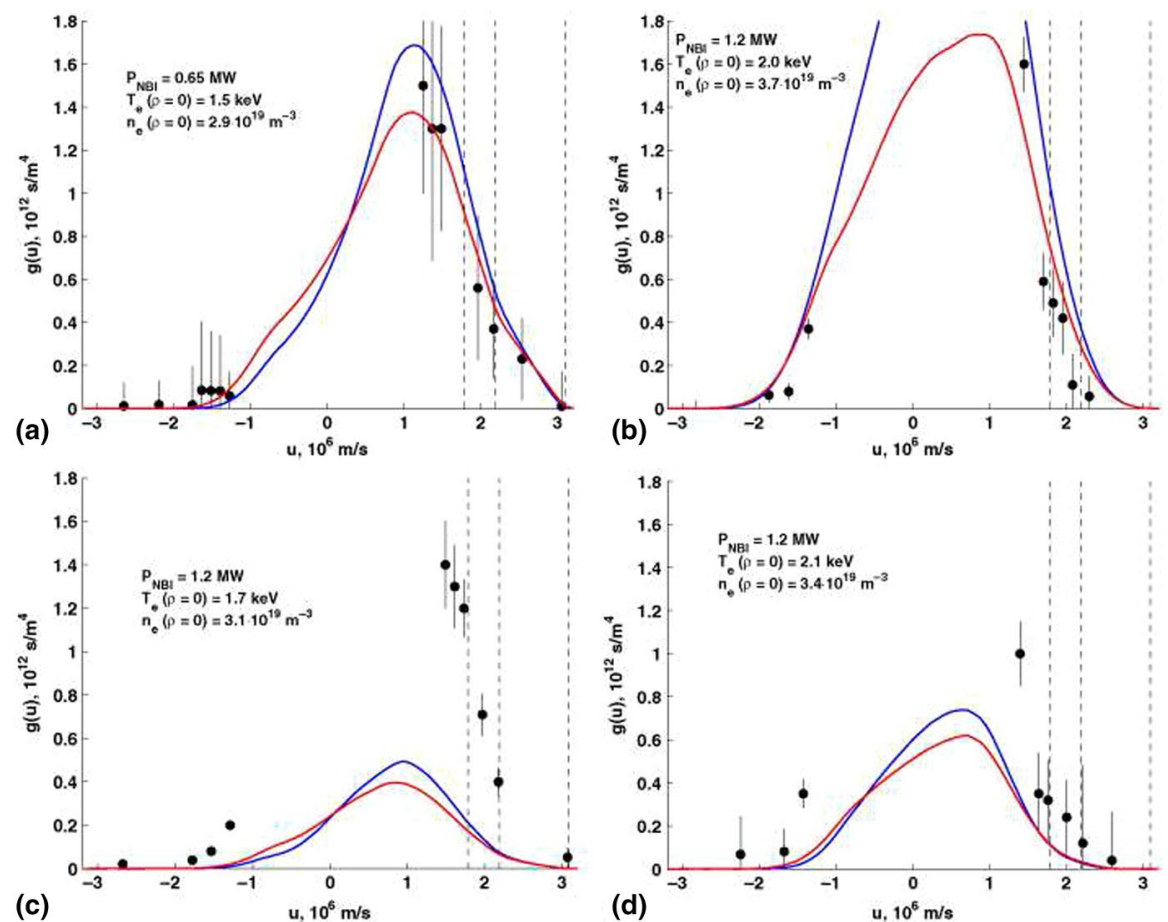

Fig. 26 Projections of the the fast-ion-velocity distribution function in TEXTOR at different radial positions and projection angles to the magnetic field. The black dots correspond to the measured values, and red and blue lines correspond to Monte Carlo simulations with ASCOT and VENUS. a, b demonstrate the results close to the plasma center; c, $\mathbf{d}$ are the results of the off-axis measurements. The figure is taken from Moseev et al. (2011)

and (d)] agreement between the CTS measurements in TEXTOR and Monte Carlo simulations by ASCOT and VENUS codes. The quantitative agreement between the measurements and the simulations is achieved in the plasma center.

The CTS diagnostic on the LHD torsatron has qualitatively confirmed the agreement between the fast-ion measurements during a heating phase with multiple NBIs and the corresponding simulation with the Monte Carlo code MORH (SEKI et al. 2010). The results are shown in Fig. 27.

The CTS diagnostic is sensitive to a variety of MHD effects, since the probing radiation scatters off fluctuations of density, magnetic field, and electric field. Fast ions are a source of free energy for a number of instabilities, such as fishbone instability, lower hybrid instability, Alfvén eigenmodes, ion cyclotron emission, etc. Some of these instabilities have a distinct footprint in the CTS spectrum and can, therefore, serve as a qualitative indicator of the presence of energetic ions (Shalashov et al. 2003; Moseev et al. 2011). 

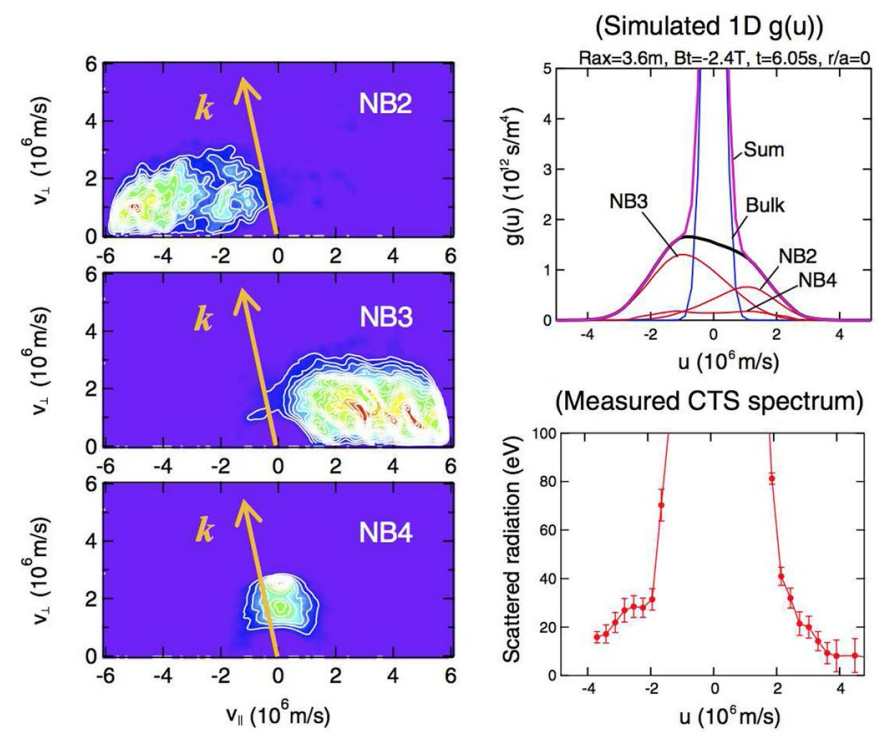

Fig. 27 Left panel: MORH simulation of the fast-ion-velocity distribution function associated with various NBIs for the LHD discharge 97496. The viewing direction of the CTS diagnostic is indicated by the $\mathbf{k}^{\delta}$ vector. Right panel: simulated projection of the fast-ion-velocity distribution function (top) and the measured CTS spectrum (bottom). The figure is taken from Nishiura et al. (2014)

\subsection{Prospects for ITER}

A feasibility study (Bindslev et al. 2004) found that CTS is capable of measuring fusion-born $\alpha$-particles in ITER both with the radial resolution of about $20-50 \mathrm{~cm}$ and with temporal resolution of about $100 \mathrm{~ms}$. However, the measurement volumes near the plasma center and on the high-field side are somewhat larger. A 1 MW gyrotron operating at $60 \mathrm{GHz}$ will be used as a source of probing radiation. The parts of the phase space which give rise to the CTS signal have been identified (Salewski et al. 2011) and the effect of the auxiliary heating on the fast-ion signal has been investigated (Salewski et al. 2009). Currently, the diagnostic is being designed with seven receiver beams overlapping the probe beam at different positions. This will allow simultaneous CTS measurements at these locations (Korsholm et al. 2016; Leipold et al. 2009). The CTS diagnostic will be located in the port plug in the equatorial port 12. A full drawer will be allocated to the CTS diagnostic at ITER. A feasibility study of isotope ratio measurements using CTS at ITER has been conducted (Stejner et al. 2012c) and conclusively demonstrated that the proposed CTS system with some minor adjustments is capable of such measurements, but until now, ITER CTS is dedicated to measurements of $\alpha$-particles and other fast ions. 


\section{Fast-ion loss detectors}

In contrast to the previous sections, where the diagnostics of confined ions are described, this section is devoted to the measurements of the lost energetic ions. Several techniques to measure MHD-induced fast-ion losses in magnetically confined fusion devices have been explored over the last decades. The most successful techniques are based on charged particle collectors. Charged particle collectors (CPC) are located at the edge of the plasma and are, therefore, exposed to a high heat load. Scintillators, Faraday cups, and activation foils have typically been used to detect the escaping ions. The diagnostics based on them have been installed in TFTR (Zweben et al. 1995, 1990; Manos et al. 1986), PLT (Murphy and Strachan 1985), Wendelstein 7-AS (Werner et al. 2001), LHD (Nishiura et al. 2004), DIIID (Fisher et al. 2010), KSTAR (Kim et al. 2012), the CHS heliotron (Kondo et al. 2000), ASDEX Upgrade (García-Muñoz et al. 2009b; Jimenez-Rey et al. 2008), JET (Darrow et al. 2004), and other fusion devices. CPCs use the magnetic field of the tokamak or stellarator to disperse the escaping ions onto the active component of the detector. Their strike points depend on the ion energy and pitch, so that such detectors provide velocity-space resolution. The temporal, energy, and pitch resolution depend ultimately on the detector active component and detailed design.

Infrared (IR) measurements are based on the heat load deposited by the escaping ions on the plasma-facing components. Fast-ion IR measurements are complicated by the thermal heat load that is typically an order of magnitude higher. Dedicated experiments allow the decoupling of thermal and suprathermal heat loads.

In JET, a post-mortem analysis of tritons and ${ }^{3} \mathrm{He}$ of the D-D fusion reaction was performed. The energy of ${ }^{3} \mathrm{He}$ was inferred from the model of the penetration depth distribution of fast ions in to the nickel target (Carruthers et al. 1990). The exposure time of several months restricts the applicability of this method. The signal in a specially designed carbon probe head for the fast triton measurements showed that the signal in it was dominated by thermalized particles.

While local measurements with CPCs normally have an excellent velocity-space coverage at just one spatial position, IR measurements have an excellent spatial coverage without velocity-space resolution. The ideal fast-ion loss detector is composed of an array of CPCs spatially distributed over the entire first wall of the device and a set of wide-angle IR cameras covering the entire first wall. To identify the MHD fluctuations responsible for the fast-ion losses, the ability to resolve signals at Alfvénic times is required. In the following, the operational principle, standard resolution, and capabilities and prospects towards future devices of a scintillator-based FILD are presented.

\subsection{Physics principle}

One of the pioneering works on detecting energy and pitch of escaping fusion products were conducted on the PLT tokamak (Murphy and Strachan 1985) using a nuclear track plastic detector (Muehling 1982). Although the nuclear track detectors 
provided energy- and pitch-resolved measurements, the main drawback was a total absence of temporal resolution. The track analysis required the removal of the detector from the tokamak for the analysis. The FILD detector on TFTR was able to overcome this particular difficulty: it used $\mathrm{ZnS}$ scintillator coupled with a video system, thus providing energy-, pitch-, and time-resolved measurements (Zweben 1989). This design became a progenitor for modern scintillator FILD systems. We explain their principles, taking the diagnostic at ASDEX Upgrade as an example.

A scintillator-based FILD (García-Muñoz et al. 2009b) acts as a magnetic spectrometer, dispersing the measured escaping fast ions onto the active component of the detector (scintillator or Faraday cup). The strike points depend on the gyroradius and on the pitch of the ions, so that FILD gives full information of the velocity space of the escaping ions at the detector position, see Fig. 28. A spatial array of detectors would then complete the phase-space information.

The main constraints for fast-ion loss detection in fusion plasmas can be clustered into two groups: first, the geometrical constraints given by the shape of the first wall and the relatively low particle radial drifts and, second, the operation constraints imposed by the pulse length and harsh environments. While the passive components of the fast-ion loss detectors (electronic, lenses, light detection systems, etc.) are installed outside of the vacuum vessel and are thus similar to those of other optical fusion diagnostics, the active components of the detector (particle detection systems) operate close to the plasma edge and can, therefore, be strongly affected by the harsh environment. A full characterization of the response of the active components of FILD detectors to the impinging ions in a fusion-relevant environment is, therefore, required to measure absolute fluxes of fast-ion losses with FILD systems. The complicated scintillator response to charged particles in a fusion-relevant environment has prevented the community to provide absolute fluxes of measured fast-ion

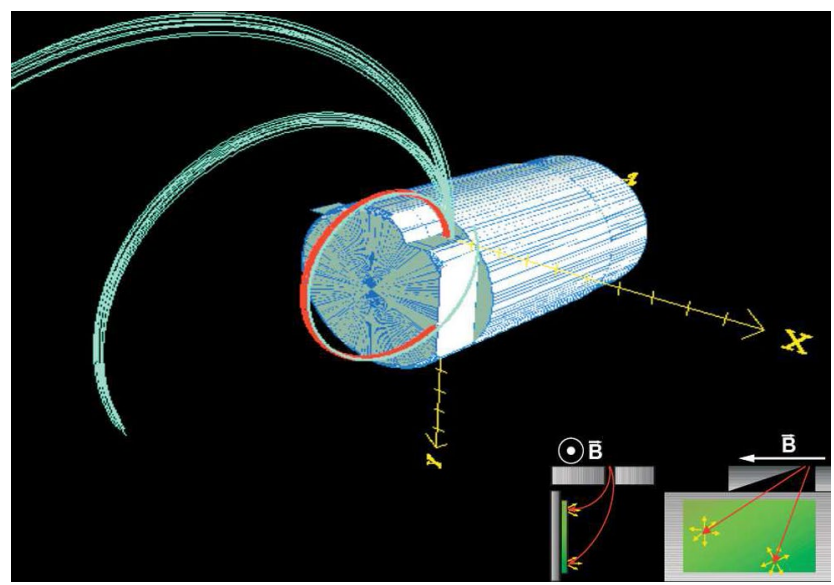

Fig. 28 FILD operational principle. CAD view of some escaping ion trajectories hitting the detector head. Particle trajectories entering the scintillator chamber are shown in blue, and particle trajectories blocked by the graphite protection in red. The inset on the right-bottom part of the figure shows the collimating process. Figure taken from García-Muñoz et al. (2009b) 


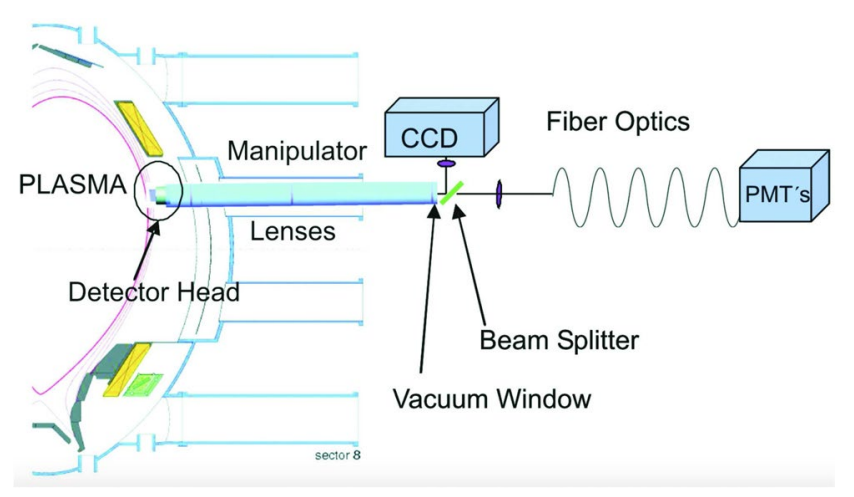

Fig. 29 ASDEX Upgrade FILD setup. Figure taken from García-Muñoz et al. (2009b)

losses. Instead, Faraday cups embedded in FILD systems have typically been used to infer absolute fluxes from the measured fast-ion losses. Background currents induced by nuclear reactions in the Faraday cup system or electromagnetic pick-up signals complicate, however, this direct measurement for a fusion plasma. The absolute calibration of an FILD scintillator screen has, nevertheless, provided some of the most valuable absolute measurements of fast-ion losses in a tokamak. An instrument function that includes the scintillator efficiency, collimator geometry, optical transmission, and camera efficiency has to be constructed for each setup.

\subsection{Instrumentation}

FILD systems contain a charged particle detector, typically a scintillator, located close to the last closed flux surface. The light emitted by the scintillator is then transmitted through an optical system and imaged by a camera and an array of photomultipliers, see Fig. 29. The scintillator screen is protected against stray radiation and other ionizing particles by a graphite cup. The geometry of this graphite cup must be well-designed to minimize secondary radiation without blocking the trajectories of the target particles. This design is typically done by means of Monte Carlo simulations that include the background magnetic field and 3D geometry of the detector head including collimator and scintillator. The simulated particle strike points on the scintillator for a given detector head geometry is then used to construct a strike map that helps identifying the energy and pitch of the escaping ion, i.e., their orbit topology. The field of view of the FILD camera and photomultiplier tube (PMT) array are optimized to cover the entire strike map, as shown in Fig. 30.

To obtain the distribution function of escaping ions hitting the aperture of the FILD head from the distribution on the scintillator, and thus the first wall, as well as the absolute flux of measured losses, an instrument function that includes the scintillator efficiency, collimator geometry, optical transmission, and camera efficiency has to be constructed (Rodriguez-Ramos et al. 2017). While the collimator factor only depends on the 3D geometry of the detector head, the scintillator efficiency depends on several parameters such as the particle species to be detected, the operation 


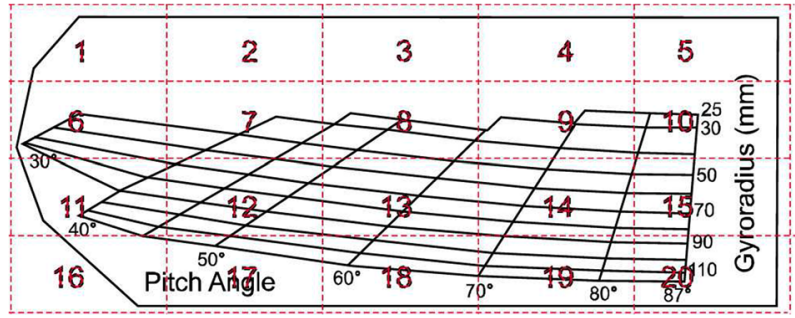

Fig. 30 Strikemap of the ASDEX Upgrade FILD systems with PMT field of view overlayed. Figure taken from García-Muñoz et al. (2009b)

temperature and the ion dose. Figure 31 shows the typical collimator factor in an ASDEX Upgrade FILD system (a), the scintillator efficiency to deuterium ions (b), and the weighting function (c). The weighting function is a convolution of the collimator factor and scintillator efficiency. The final instrument function is obtained from the actual measurement, the weighting function, the optical transmission, and the efficiency of the light acquisition systems. Figure 32 shows the absolute flux of measured fast-ion losses in velocity space by an FILD system at the ASDEX Upgrade tokamak.

\subsection{FILD measurement of MHD-induced fast-ion losses}

MHD-induced fast-ion losses have been measured by FILD systems in virtually all major fusion devices, such as NSTX (Darrow et al. 2013), CHS (Shinohara et al. 2007), and ASDEX Upgrade. MHz sampling rates are used to identify the MHD fluctuations responsible for the observed fast-ion losses. While CCD cameras are used to obtain the velocity-space of the escaping ions with high resolution but moderate (ms) temporal resolution, the PMT array is used to identify the fluctuations responsible for the losses through Fast Fourier Transforms (FFT). Typical measurements of fast-ion losses induced by AEs in the ASDEX Upgrade tokamak are shown in Figs. 33 and 34.

Figure 33 shows the velocity space of the measured AE-induced fast-ion losses in an ICRF-heated plasma at two different time points; in the presence of many AEs (a) and in the presence of a single AE (b). This information is used to calculate the orbit topology of the lost ion backwards in time to understand the wave-particle interaction that has caused this loss.

This accurate velocity-space measurements are complemented with PMT based time-resolved measurements of the ion flux. These measurements not only allow the identification of the most deleterious MHD fluctuations for the fastion confinement, but they also help to understand the wave-particle interactions underlying the observed fast-ion transport. Figure 34a shows a spectrogram of an FILD signal obtained in a plasma with AE-induced fast-ion losses. TAEs and Reversed Shear AEs (RSAEs) are clearly visible in the FILD spectrogram. The raw data shown in Fig. 34b show, however, two different components in the FILD signals: a fluctuating signal, that produces the spectrogram of Fig. 34a and 

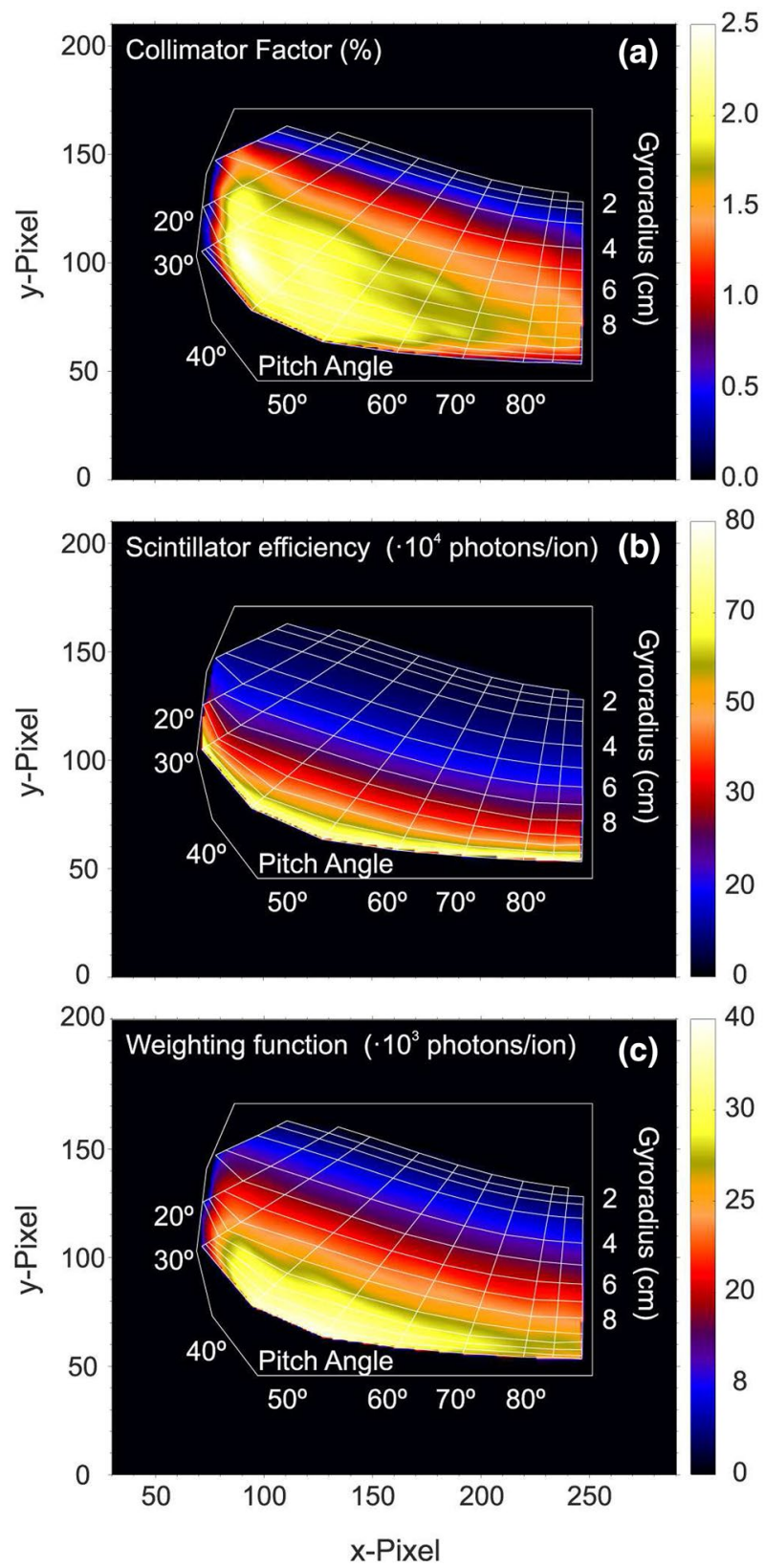

Fig. 31 a Collimator factor, b scintillator efficiency and $\mathbf{c}$ weighting function for the ASDEX Upgrade FILDs. Figure taken from Rodriguez-Ramos et al. (2017) 


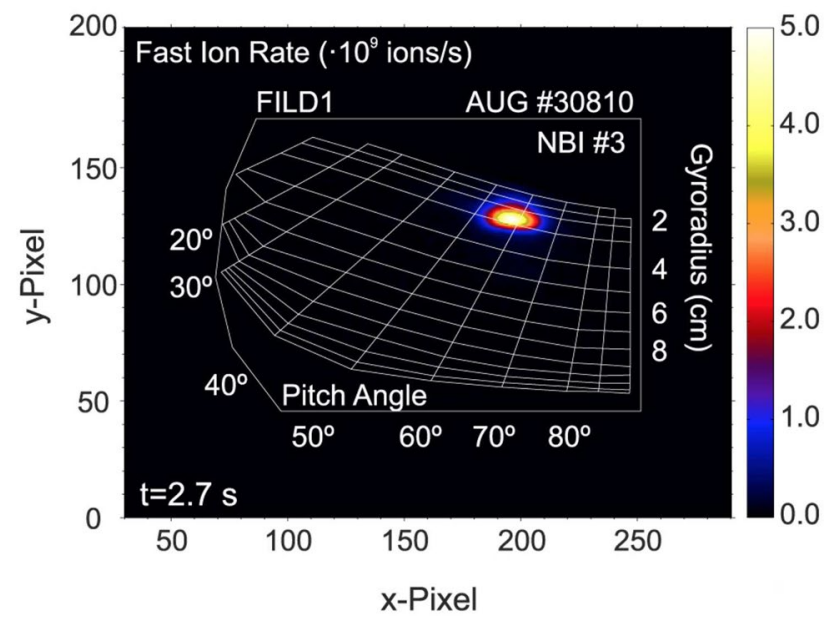

Fig. 32 Absolute fast-ion loss rate on the FILD scintillator screen. Figure taken from Rodriguez-Ramos et al. (2017)
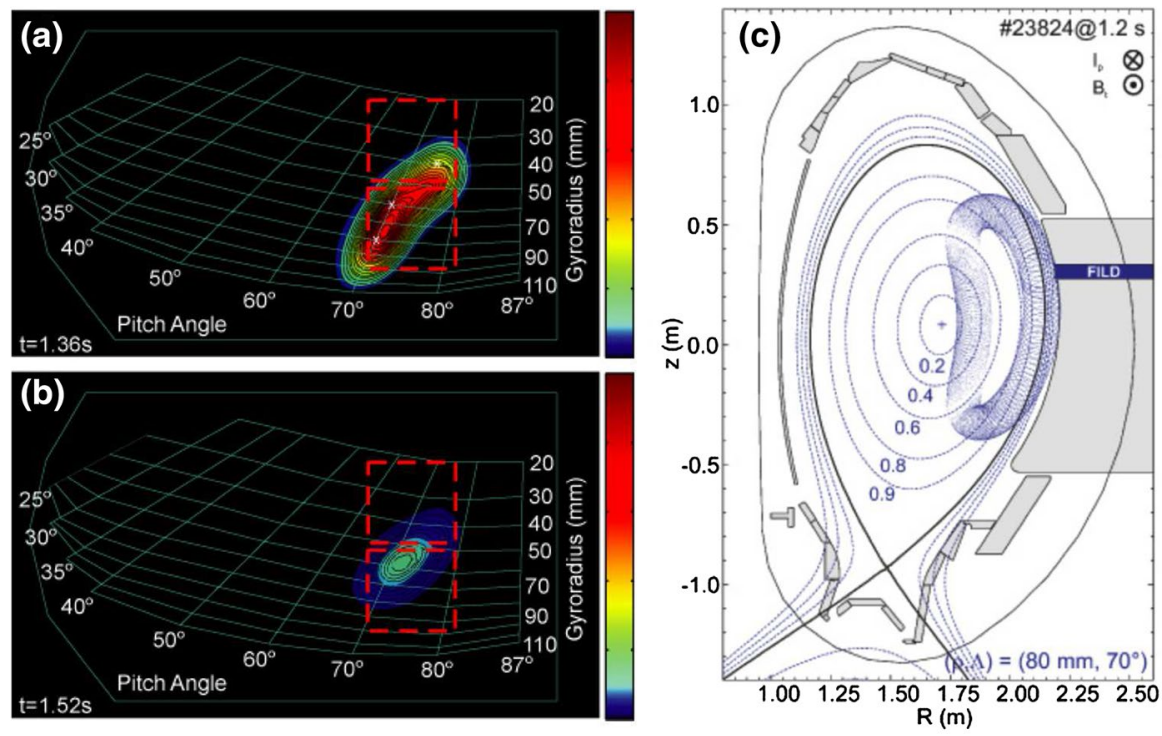

Fig. 33 a Velocity space of escaping ions in the presence of (a) many AEs and b a single $n=5$ AE. c Typical trajectory of a lost hydrogen ion with $E=200 \mathrm{keV}$ calculated backward in time from detector collimator to the plasma. The vertical black line indicates the position of the ICRF-heating resonance layer. Figure taken from García-Muñoz et al. (2010a)

a fairly constant signal. Extensive data analysis and theoretical efforts aimed to estimate the convective and diffusive character of MHD-induced fast-ion losses based on such kind of FILD measurements. 


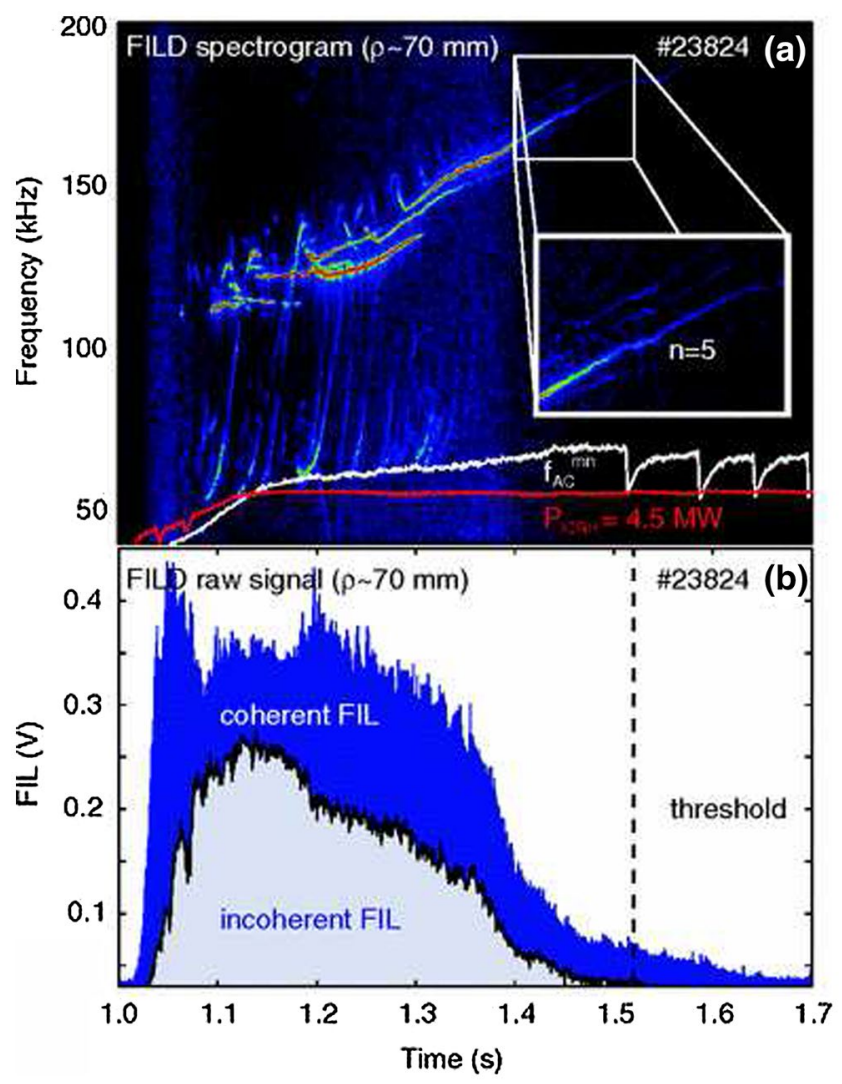

Fig. 34 a Power spectrogram of the fast-ion loss signal with gyroradius $70 \mathrm{~mm}$. The inset shows the single TAE responsible for the onset of the incoherent losses. b Fast-ion loss signal. The coherent and incoherent components of the losses are highlighted. The vertical dashed line depicts the threshold for the incoherent losses. Figure taken from García-Muñoz et al. (2010b)

\subsection{Prospects for ITER}

Although several diagnostics for confined fast ions are being proposed for ITER, a lost $\alpha$-particles diagnostic has not been approved as of yet. The harsh environment in ITER - a nuclear machine-places a number of constraints on standard fast-ion loss detection techniques unprecedented on present tokamaks with easier access and more tolerable conditions. On the basis of the physics requirements and integration capabilities, the Port Plugs and Diagnostics Integration Division at ITER Organization has started to undertake a conceptual study of a reciprocating FILD system for ITER (García-Muñoz et al. 2016).

ASCOT simulations have been carried out to estimate the fast-ion flux on the ITER first wall. Externally applied 3D fields have been used to simulate the effect of MHD fluctuations on $\alpha$-particle transport and thus to estimate the detector signals 
in the presence of MHD fluctuations. The optimal detector position and measurement cycle are, in fact, given by the balance between the measured fast-ion signals and the heat load on the detector head. Based on these ASCOT simulations, a stroke of $20 \mathrm{~cm}$ is sufficient to keep the entire detector head behind the Diagnostic First Wall (DFW) in a safe parking position as well as to deploy the detector aperture $11 \mathrm{~cm}$ outside of the DFW during measurements. In the present design, the scintillator plate is located approximately $2 \mathrm{~cm}$ behind the heat shield. Figure 35 shows the expected $\alpha$-particle heat load on the ITER first wall. A total number of $10^{6}$ test fusion-born $\alpha$-particles has been followed until they thermalize or hit the wall. The different wall structures on the low-field side are clearly visible together with the $n=4$ RMP structure. As expected, due to the ion grad-B drift, most losses appear at the divertor and mid-plane wall structures with a maximum heat load around 1 $\mathrm{MW} / \mathrm{m}^{2}$. The $n=4 \mathrm{RMP}$, caused by the ELM mitigation coils, has $I_{\text {coil }}=90 \mathrm{kA}$, has been used to simulate an extreme case with high but realistic $\alpha$-particle losses. Detailed heat load analysis including transient Edge Localized Modes (ELMs) losses and nuclear heating has shown that, at the measurement position, the detector head will be exposed to heat loads similar to that in the present large tokamaks. The nuclear heating suffered by the system during the entire discharge forces the design to include an active cooling system. The high neutron and gamma fluxes expected close to the ITER first wall lead also to background scintillator emission that could, if not designed properly, hamper the FILD measurements at ITER.

Preliminary calculations show that the MHD-induced $\alpha$-particle signals expected in ITER are well above the background signals produced by neutron and gamma fluxes. Figure 36 shows a synthetic measurement of $\alpha$-particle losses induced by an $\mathrm{n}=4$ externally applied RMP including background emission produced in the scintillator by charged particles born in nuclear reactions.

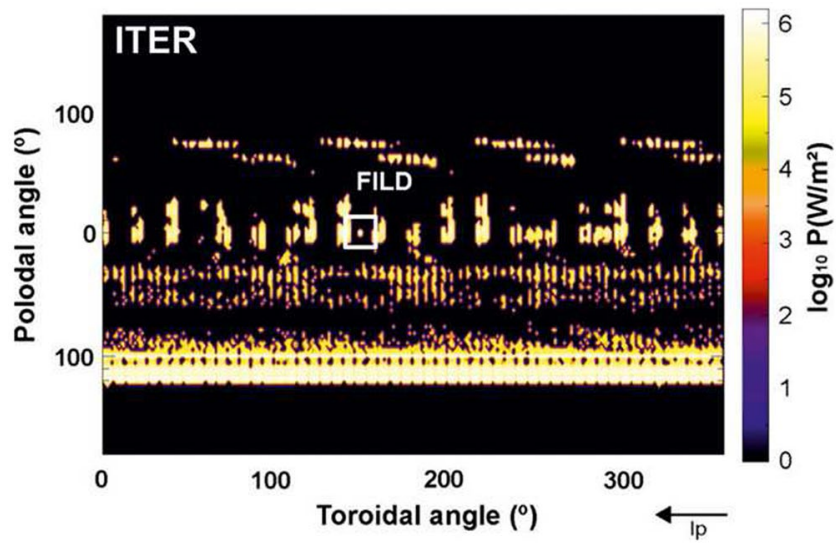

Fig. 35 ASCOT simulations of $\alpha$-particle heat load in ITER on a 3D first wall due to an externally applied $n=4$ RMP. The location of the FILD head is indicated with a white box. Figure taken from García-Muñoz et al. (2016) 


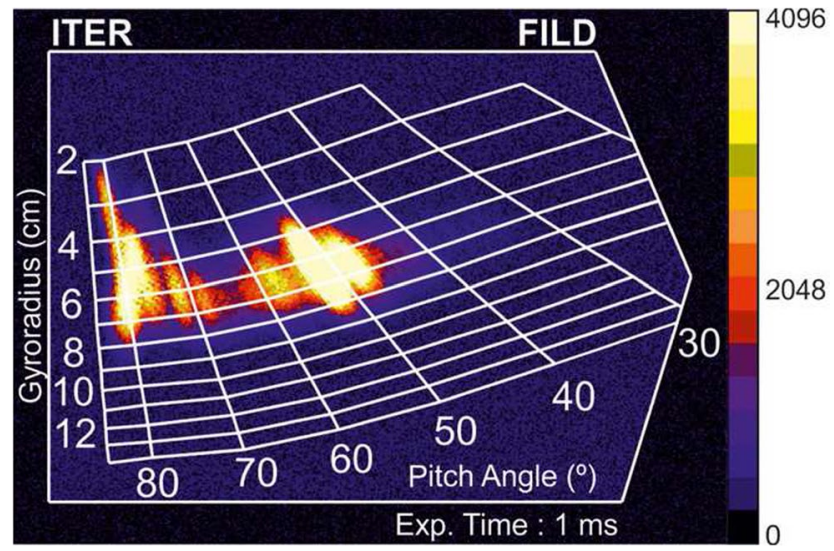

Fig. 36 Synthetic measurement of $\alpha$-particle losses induced by RMPs with the present design of the ITER FILD. Figure taken from García-Muñoz et al. (2016)

\section{Measurements of fast-ion-velocity distribution functions: from velocity-space sensitivity to tomography}

The traditional way to analyze fast-ion measurements is to compare the actual measurements with synthetic measurements based on numeric simulations. Examples for this method abound in the literature for CTS (Salewski et al. 2010; Moseev et al. 2011; Nielsen et al. 2015; Rasmussen et al. 2015), FIDA (Heidbrink et al. 2004, 2007; Geiger et al. 2015b), NES (Hellesen et al. 2010, 2013; Eriksson et al. 2015; Schneider et al. 2016) and GRS (Kiptily et al. 2010; Tardocchi et al. 2011; Nocente et al. 2012b). Agreement between synthetic measurements and actual measurements is taken as indication that the simulated fast-ion phase-space distribution function is a good model for the fast-ion population in the plasma. However, often, measurements and simulations disagree. In this case, it is difficult to tell from the measurements what causes the discrepancies and what this actually means for the fast-ion phase-space distribution function. In this section, we discuss methods to connect fast-ion measurements with the fast-ion-velocity distribution function.

\subsection{Velocity-space sensitivity of fast-ion diagnostics}

The first step to learn about the velocity distribution function is to assess the velocity-space sensitivity of the various fast-ion diagnostics. This idea was developed as a qualitative analysis tool to understand a puzzling observation in an experiment at DIII-D (Heidbrink et al. 2007). The FIDA measurements and the neutron count measurements behaved in a very similar way, whereas the NPA measurements behaved qualitatively differently. This was at the time surprising, because the NPA and FIDA monitor neutrals generated in the same charge-exchange reaction. FIDA measures the D- $\alpha$ light emitted by the neutrals, and NPA measures the neutrals that do not re-ionize on the path to the detector. Therefore, the NPA signal 
was (mistakenly) expected to behave in a qualitatively similar way as the FIDA signal. However, the velocity-space observation region of FIDA and the neutron count measurement are broad regions in velocity space, and they are fairly similar, whereas the NPA observes a tiny region in velocity space which was illustrated by so-called weight functions. This new understanding of the velocity-space observation regions then resolved this at the time puzzling observation. Since then, weight functions have been developed for all major fast-ion diagnostics: FIDA (Heidbrink et al. 2007; Salewski et al. 2014a), neutral particle analyzers (NPA) (Heidbrink et al. 2007), CTS (Salewski et al. 2011), fast-ion loss detectors (Pace et al. 2012; GaldonQuiroga et al. 2018), NES (Jacobsen et al. 2014, 2017, 2015), and GRS (Salewski et al. 2015a, 2016a).

For these diagnostics, the weight functions, $w$, relate measurements, $s$, to $2 \mathrm{D}$ fast-ion distribution functions, $f$, by the integral equation: (Heidbrink et al. 2007; Heidbrink 2010; Salewski et al. 2011, 2014a; Jacobsen et al. 2014, 2017, 2015; Salewski et al. 2015a, 2016a)

$$
s\left(m_{1}, m_{2}, \phi\right)=\int_{\text {vol }} \int_{0}^{\infty} \int_{-\infty}^{\infty} w\left(m_{1}, m_{2}, \phi, v_{\|}, v_{\perp}, \mathbf{x}\right) f\left(v_{\|}, v_{\perp}, \mathbf{x}\right) \mathrm{d} v_{\|} \mathrm{d} v_{\perp} \mathrm{d} \mathbf{x} .
$$

$s\left(m_{1}, m_{2}, \phi\right)$ is the detected signal in the measurement range $m_{1}<m<m_{2}$ with a viewing angle $\phi$ between the line-of-sight of the diagnostic and the magnetic field. $\left(v_{\|}, v_{\perp}\right)$ are the velocities parallel and perpendicular to the magnetic field, respectively, and $\mathbf{x}$ describes the spatial coordinates. The units of $f$ in Eq. 12 are $\left(\mathrm{s}^{2} / \mathrm{m}^{5}\right)$. The units of weight functions are thus (signal/fast ion), where the units of the signal are particular to each instrument.

Weight functions are routinely found numerically for any diagnostic using a forward model that predicts the measured signal for an arbitrary fast-ion distribution function. In this numerical approach, the signal $s$ due to $N_{f}$ fast ions is calculated for a grid of velocities $\left(v_{\|}, v_{\perp}\right)$ or $(E, p)$ covering the target velocity-space region. The velocity distribution function of the $N_{f}$ fast ions at phase-space position $\left(\mathbf{x}_{0}, v_{\| 0}, v_{\perp 0}\right)$ is

$$
f\left(v_{\|}, v_{\perp}, \mathbf{x}\right)=N_{f} \delta\left(v_{\|}-v_{\| 0}\right) \delta\left(v_{\perp}-v_{\perp 0}\right) \delta\left(\mathbf{x}-\mathbf{x}_{0}\right) .
$$

Substitution into Eq. 12 and integration shows that the amplitudes of weight functions at phase-space position $\left(\mathbf{x}_{0}, v_{\| 0}, v_{\perp 0}\right)$ are readily computed from

$$
w\left(m_{1}, m_{2}, \phi, v_{\| 0}, v_{\perp 0}, \mathbf{x}_{0}\right)=\frac{s\left(m_{1}, m_{2}, \phi\right)}{N_{f}} .
$$

Weight functions hence show the integrated signal between two spectral points per ion at phase-space position $\left(\mathbf{x}_{0}, v_{\| 0}, v_{\perp 0}\right)$. The shapes of the weight functions of the various diagnostics have been understood by considering the underlying physical processes for each diagnostic (Salewski et al. 2011, 2014a; Jacobsen et al. 2015; Salewski et al. 2015a, 2016a). In velocity space $\left(v_{\|}, v_{\perp}\right)$, FIDA and CTS observe triangular regions, whereas NES and one-step reaction GRS observe regions bounded 


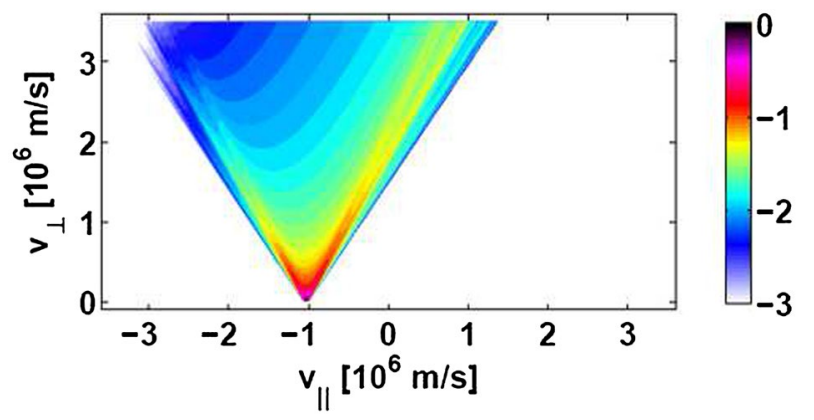

Fig. 37 Theoretical model of an FIDA weight function which looks similar to the CTS weight function from Fig. 38. The triangular shape comes from the Doppler shift. The bias towards the right comes from changing charge-exchange probabilities along the gyro-orbit. The fringing at the boundaries comes from Stark splitting. The figure is taken from Salewski et al. (2014a)

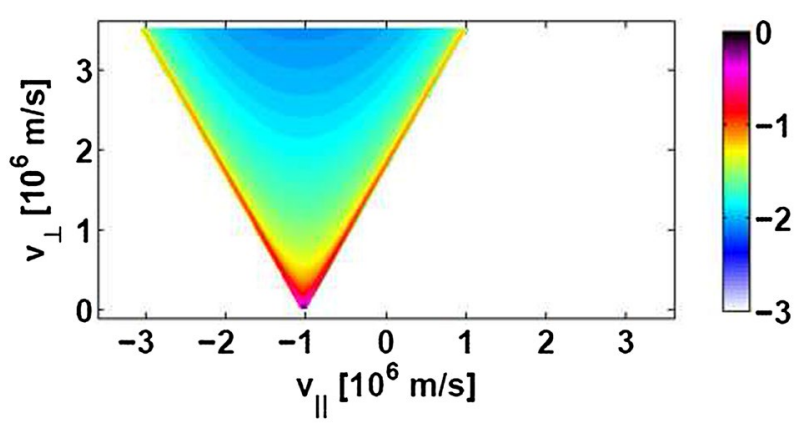

Fig. 38 CTS weight functions have a triangular shape due to the Doppler shift. The opening angle is 2 $\phi$, and the closest distance to the origin is the projected velocity $u$.) The figure is taken from Salewski et al. (2014a)

by circular arcs. Two-step reaction GRS observes fairly complicated regions, whereas NPA observes tiny regions. Examples of weight functions for the major fast-ion diagnostics are illustrated in Figs. 37, 38, 39, 40, 41, 42.

This understanding of velocity-space sensitivities allows five types of data analysis. First, the observable and the unobservable velocity space can be told apart. Second, given a 2D fast-ion-velocity distribution function, the velocity distribution of the ions generating a given measurement can be calculated. Third, given the absence of a measurement signal, the corresponding empty region of velocity space can be identified. Fourth, synthetic measurements can be calculated rapidly. Fifth, we can infer 2D fast-ion-velocity distribution functions by velocity-space tomography which we will discuss in the next section. 
Fig. 39 NPA weight function covers a small region in velocity space, since the the gyrophase is fixed to a small range by the geometry of the measurement. The figure is taken from Heidbrink et al. (2007)
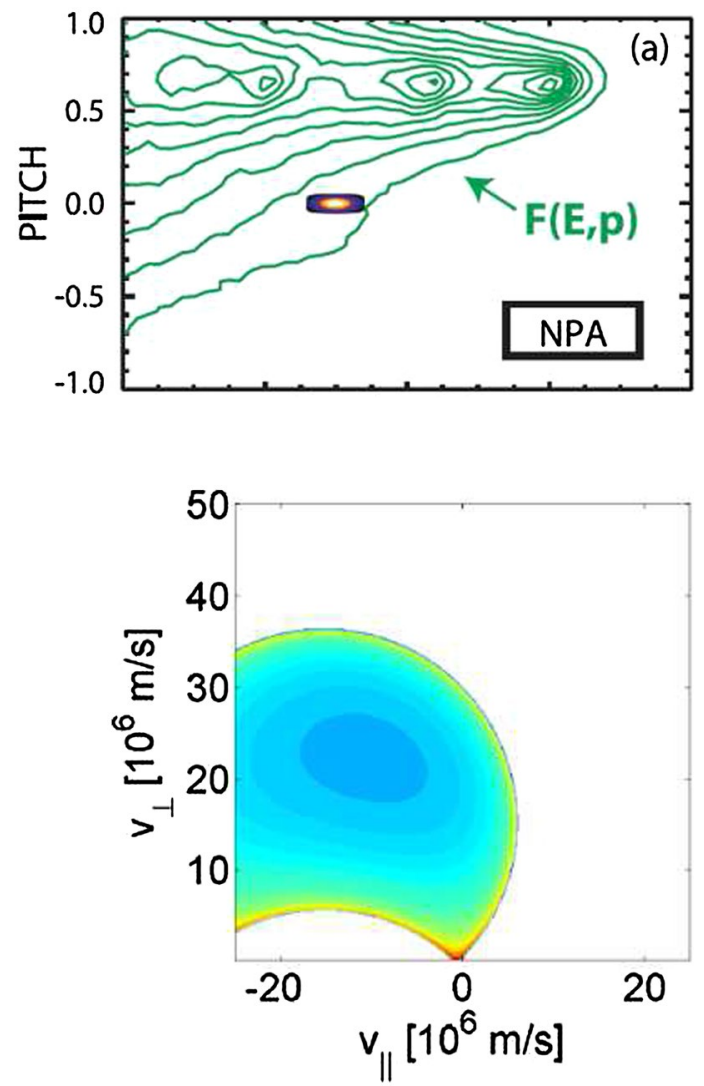

Fig. 40 NES weight functions are bounded by circular arcs. Their shape is determined by conservation of energy and momentum. Here, the line-ofsight is oblique with respect to the magnetic field. The figure is taken from Jacobsen et al. (2015)

Fig. 41 One-step reaction GRS weight functions for four different Doppler shifts for an oblique LOS. This type of weight function has energy resolution as the form of the weight function is close to concentric circles. The figure is taken from Salewski et al. (2016a)

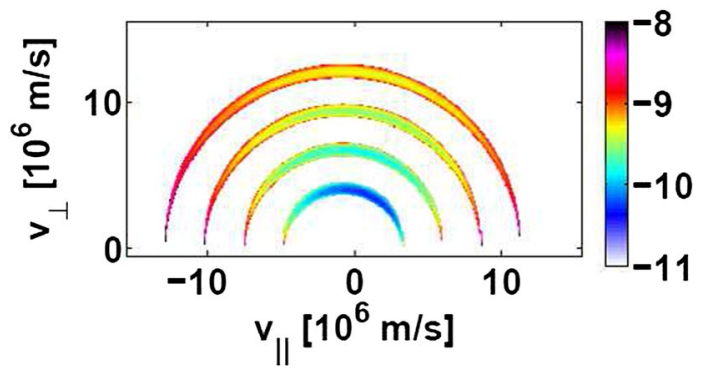

\subsection{Velocity-space tomography and integrated data analysis of fast-ion diagnostics}

Velocity-space tomography is a method to obtain a 2D image of the fast-ion-velocity distribution function based on the available fast-ion measurements (Egedal and Bindslev 2004; Salewski et al. 2011, 2012, 2013, 2014b, 2015b, 2016b, 2017, 2018; Geiger et al. 2015a; Weiland et al. 2016; Jacobsen et al. 2016a, b; Jaulmes et al. 


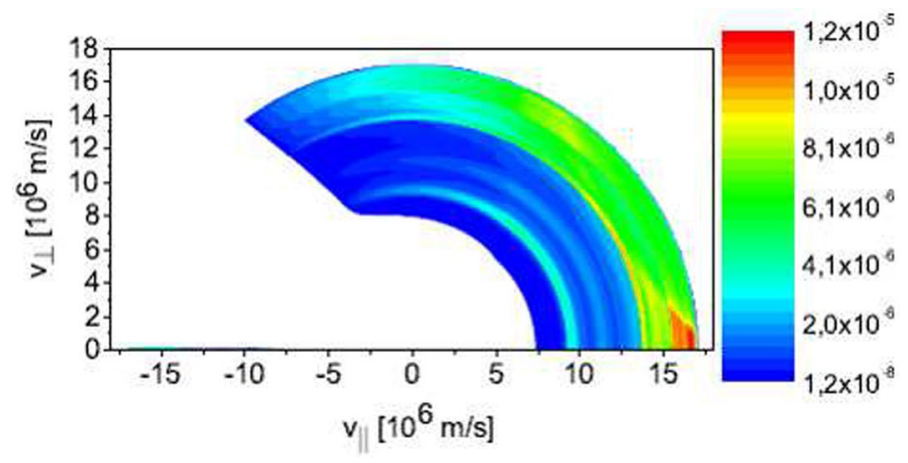

Fig. 42 GRS weight function for two-step reactions for an oblique LOS. The figure is taken from Salewski et al. (2015a)

2016; Rasmussen et al. 2016). This 2D image is the best useful fit to all measurements from the available diagnostics. The spectra contain often hundreds of data points, and this large amount of data is difficult to exploit by traditional means, also considering that each data point observes a different region in velocity space. Furthermore, the data are usually presented in popular diagnostic units that are difficult to interpret for outsiders to the particular diagnostic, such as experts from other fast-ion diagnostics or the simulation community. The $2 \mathrm{D}$ image produced by velocity-space tomography, on the contrary, is straightforward to interpret and shows directly the fast-ion-velocity distribution function which is the fundamental quantity of interest that is known to most workers. Another advantage of this inversion method is that nuisance parameters are accounted for, whereas the diagnostic spectra also depend on nuisance parameters. For example, the amplitude of measured spectra during a sawtooth crash decreases if the ion density decreases. The method has become possible due to the weight function formalism discussed in the previous section. The tomographic inversion of the fast-ion measurements can be directly compared with numerical simulations and hence provides an alternative meeting ground between theory and observation.

Discretization of Eq. 12 allows us to reformulate the forward model of a given fast-ion diagnostic as the matrix equation:

$$
S=W F,
$$

where $F$ is the fast-ion-velocity distribution function rearranged as column vector, $S$ is a column vector holding the measurements, and $W$ is a matrix holding the weight functions rearranged as rows (Salewski et al. 2012). To take the measurement uncertainty of the individual data points into account, both sides of each line of the equation system are divided by the uncertainty. This step also allows the combination of various diagnostics as it normalizes the $S$ vector to become an SNR vector, where all entries have similar magnitudes which improves the conditioning of the problem (Salewski et al. 2013). The problem to determine $F$ from $S$ is now to find a useful inversion of $W$. Due to noise in the measurements, however, this problem has no solution, and instead, one computes a least-squares fit. As the weight matrix $W$ is 
ill-conditioned, noise in the measurements would be strongly amplified in the calculation of $F$ from $S$. This makes it necessary to add some form of regularization to the problem as is done in the standard position-space tomography. To date, velocity-space tomography has employed the standard inversion methods singular value decomposition (SVD), the maximum entropy method, and variants of the Tikhonov regularization (Salewski et al. 2014b, 2015b; Weiland et al. 2016; Jacobsen et al. 2016a; Salewski et al. 2016b).

Compared with many other tomography applications, the amount of measurement data is small due to the limited optical access to the plasma and the often comparatively small SNR. Inversions are for this reason often plagued by artefacts. Artefacts can be reduced by optimizing the discharges. For FIDA, usually, L-mode plasmas with low density and low heating power work well (Salewski et al. 2014b; Geiger et al. 2015a; Weiland et al. 2016; Jacobsen et al. 2016a). The installation of additional fast-ion diagnostics is a very effective remedy against artefacts (Salewski et al. 2012; Weiland et al. 2016). High-definition tomography techniques make use of additional prior information one might have which also decreases artefacts and improves the inversions (Salewski et al. 2016b). Potent prior information are the measured absence of ions, the position of the injection sources in velocity space, the non-negativity constraint, the smoothness of the velocity distribution function, or, if available, a numeric simulation of the discharge (Salewski et al. 2016b). These high-definition tomography techniques improved results for the five-view FIDA diagnostic at the tokamak ASDEX Upgrade and further allow the use of inversion techniques for more common FIDA systems with two or three views. The recent inversion of GRS and NES measurement data at JET also relied on these techniques (Salewski et al. 2017). As an example, we show the formulation as a Tikhonov problem with non-negativity constraint:

$$
F^{*}=\arg \min _{F}\left\|\left(\begin{array}{l}
W \\
\lambda L
\end{array}\right) F-\left(\begin{array}{l}
S \\
0
\end{array}\right)\right\|_{2} \text { subject to } F^{*} \geq 0 .
$$

Here, the upper row minimizes the residual of the original least square problem. The lower row penalizes the size of $L F$, where $L$ is the regularization matrix which is often chosen to effect a gradient in discrete form. This penalty operator is often used in tomography applications and encodes our belief that the velocity distribution function is smooth, which is a form of prior information. The regularization parameter $\lambda$ must be found as part of the solution and balances the requirement to fit the data and the smoothness requirement. The combination of fast-ion diagnostics has been demonstrated using FIDA and CTS (Salewski et al. 2013; Jacobsen et al. 2016b; Rasmussen et al. 2016) and GRS and NES (Salewski et al. 2017).

\subsection{Experimental results of velocity-space tomography}

Velocity-space tomography has provided measurements of NBI- and ICRF fastion-velocity distribution functions. Good agreement between the measured 
fast-ion-velocity distribution functions and TRANSP simulations was found for an NBI-heated plasma at ASDEX Upgrade in the absence of strong MHD activity (Salewski et al. 2014b; Weiland et al. 2016). Profiles of the fast-ion-velocity distribution function from the plasma center towards the edge have been measured at ASDEX Upgrade (Weiland et al. 2017). The method has also recently revealed velocity-space redistribution patterns of fast ions due to sawtooth crashes at ASDEX Upgrade (Geiger et al. 2015a; Weiland et al. 2016; Jacobsen et al. 2016a, b; Salewski et al. 2016b; Jaulmes et al. 2016; Rasmussen et al. 2016). Measured velocity distribution functions in the plasma center at ASDEX Upgrade before and after a sawtooth crash are illustrated in Fig. 43.

The zeroth moment of the fast-ion-velocity distribution function provides the fast-ion density which is thought to be an important parameter. The ITER measurement requirements for fast-ion diagnostics ask for measurements of the fastion or $\alpha$-particle densities and energy spectra. These important parameters can be measured by velocity-space tomography without assuming any model for the fastion-velocity distribution function, such as a slowing-down distribution. Figure 44 compares measured time traces of the fast-ion density in a sawtoothing ASDEX Upgrade plasma with a corresponding TRANSP simulation. The TRANSP simulation predicts somewhat more violent sawtooth crashes than the measurements. Velocity-space tomography further allows us to study phase-space densities such as the densities in different pitch ranges which is also illustrated in Fig. 44. A qualitative difference appears for pitches $p<0.25$, where no evidence for sawteeth is found in the FIDA data. However, the TRANSP simulation does predict sawteeth in this pitch range as for the other pitches. The rich FIDA data in this discharge could be efficiently summarized in a movie of the velocity distribution function measured by velocity-space tomography which is available as supplementary material to reference Salewski et al. (2016b). The time resolution of the movie was $2 \mathrm{~ms}$.

Energetic ions in the $\mathrm{MeV}$ range have been studied at JET by velocity-space tomography. The energetic ions were generated by $3 \mathrm{MW}$ of ICRF at the third harmonic of deuterium and 4.5 MW of NBI. The ICRF accelerates the NBI ions from below $120 \mathrm{keV}$ to $2 \mathrm{MeV}$. Measurements were done with a HpGe GRS detector providing spectral resolution of two gamma-ray peaks as well as three NES detectors: TOFOR, a single-crystal diamond detector, and a liquid scintillator detector. Figure 45 shows a comparison of the tomographic inversion of these measurements and a numerical simulation. The length and width of the fast-ion tail were found to be in excellent agreement including the presence of the barrier region that does not permit acceleration to energies larger than $2 \mathrm{MeV}$ (Schneider et al. 2016) and the thickening of the tail towards lower velocities due to collisions. Velocity-space tomography of ICRF acceleration of NBI ions above the injection energy was also recently demonstrated at ASDEX Upgrade (Weiland et al. 2017). 
Fig. 43 Measurements of the fast-ion-velocity distribution function in the plasma center at ASDEX Upgrade discharge \#32323 before and after a sawtooth crash (Salewski et al. 2016b)

Fig. 44 Measured and simulated fast-ion densities in AUG \#32323 (Salewski et al. 2016b)

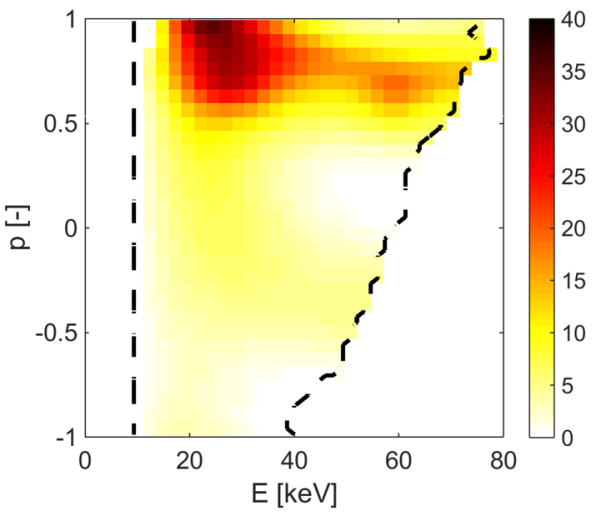

(a) Before

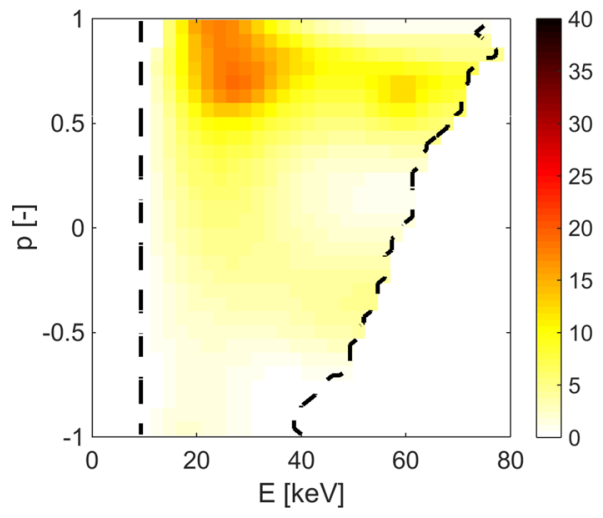

(b) After

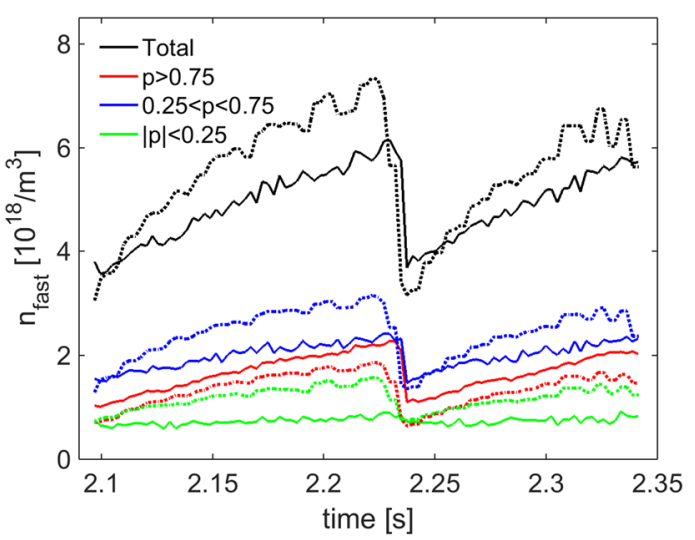


Fig. 45 Measurement and simulation of an MeV-range ionvelocity distribution function at JET (Salewski et al. 2017)

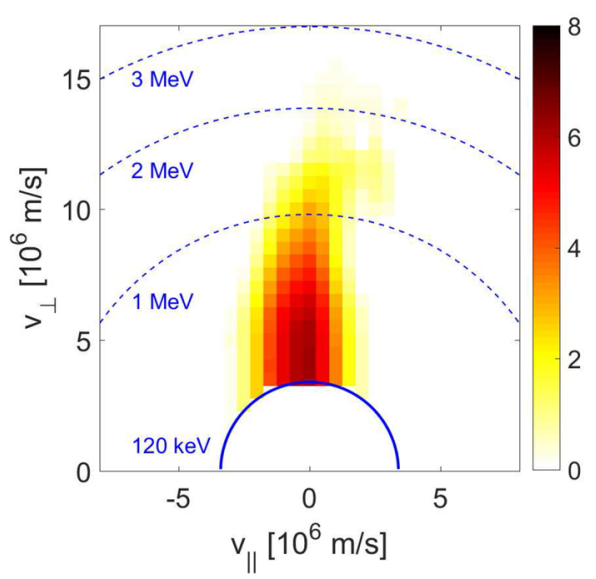

(a) Measurement

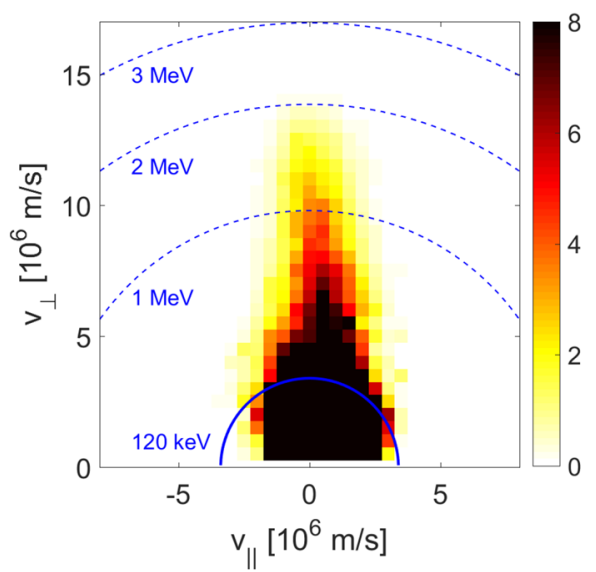

(b) Simulation

\section{Conclusions}

Fusion devices are equipped with a number of diagnostics probing various parts of the fast-ion phase-space distribution function. Key fast-ion diagnostics are NES, GRS, CTS, FIDA, NPA, and FILD. Confined fast ions are difficult to diagnose due to the limited access to the plasma and the usually low SNR. Nevertheless, recent advances in the various diagnostic methods have allowed fast-ion measurements with unprecedented levels of detail, allowing us to validate and confront our understanding of fast-ion physics.

Both NES and GRS diagnostics measure the products of nuclear reactions which only occur if at least one of the reactants is energetic. Neutron diagnostics can be divided into the neutron counters and neutron spectrometers. Neutron counters are compact and can be installed at several locations which allows a generally accurate 
reconstruction of the emission profiles. High-resolution neutron spectrometers are bulky, but the size is necessary to retrieve accurate information that is required to study the suprathermal populations of the fuel ions. The most successful instruments are based either on time-of-flight or magnetic proton recoil techniques. Recently developed diamond detectors allow compact neutron spectrometers in the future, but now the quality of the data from conventional neutron spectrometers is superior.

GRS is based on two different types of reactions: one- and two-step reactions. One-step reactions have smaller cross sections than two-step reactions and, therefore, often have a fairly low SNR. The two-step reaction between $\alpha$-particles and beryllium is foreseen as workhorse for $\alpha$-particle measurements at ITER. High-resolution GRS measurements now make it possible to resolve the shapes of individual gamma-ray peaks. The Doppler broadening of the peaks is related to the ion motion in the plasma. Modern materials for GRS are $\mathrm{LaBr}_{3}$ which allows good energy resolution at $\mathrm{MHz}$ counting rates and $\mathrm{HpGe}$ which allows $\mathrm{keV}$ energy resolution at moderate counting rates. Both NES and GRS will be installed on ITER, where they benefit from increased neutron and gamma-ray fluxes, thanks to the larger size and higher fusion performance of this machine. The design of the setup is similar to the one used at JET now.

Active NPA and FIDA are based on charge-exchange reactions. Both diagnostics rely on substantial densities of neutral atoms in the plasma core. NPA measures escaped neutrals from the plasma that are born in the charge-exchange process between fast ions and injected neutral atoms. The diagnostic has a very good SNR and extremely high dynamic range, over six orders of magnitude in some devices. The spatial resolution and in particular the velocity-space resolution of active NPAs is very good. FIDA is based on a spectroscopy of the broadened Balmer-alpha line in the same charge-exchange reaction. The diagnostic allows localized measurements from different lines-of-sight which allows the reconstruction of 2D velocity distribution functions. In addition, profile measurements are possible by an array of lines-of-sight. While the diagnostic works very well in low-density plasmas with ion energies below $200 \mathrm{keV}$, the diagnostic of MeV-range ions is hampered by the very low charge-exchange cross sections at high energies. High-density plasma further leads to a poor beam penetration and high levels of bremsstrahlung which makes an implementation at ITER challenging.

Information on the projection of the fast-ion-velocity distribution function is encoded in the scattering spectrum of the CTS diagnostic. The diagnostic in modern machines is flexible and allows spatially resolved measurements with different resolved angles. Besides the information on fast ions, the scattering signal contains signatures of the MHD activity and various waves in the plasma. This makes the inference of the fast-ion-velocity distribution function challenging. Often, long integration times are needed to receive a spectrum with good SNR. The diagnostic is planned for ITER, where it will probe fusion-born and NBI-originated fast ions at seven spatial locations.

Fast-ion loss detectors are used for the measurements of lost energetic ions. They are capable of resolving the loss in the phase space, they have high dynamic range and sensitivity, thus allowing measurements with high temporal resolution. The information provided by FILD systems has been key to understand the wave-particle 
interactions responsible for the observed MHD-induced fast-ion losses in present devices. Preliminary studies for the integration of a FILD system in ITER are quite encouraging though further work is still necessary.

Currently, there is no diagnostic alone which is capable of resolving the entire confined fast-ion phase-space distribution function. However, the measurement signals of the various diagnostics can be related to the phase-space distribution function by so-called weight functions. The analysis of weight functions shows how diagnostics complement each other. They are used for installing new diagnostics for maximizing coverage of the fast-ion phase space by the entire set of diagnostics on a given machine. This allows an integrated data analysis of the available fastion diagnostics by formulating an inversion problem that can be solved by standard methods of usual position-space tomography. Using prior information, the velocity distribution function can now be reconstructed by velocity-space tomography. A combination of confined fast-ion diagnostics is used for the reconstruction of the velocity distribution function on ASDEX Upgrade and most recently on JET. Efforts to implement similar approaches on other machines and extensions to 3D are on the way. Joint experiments within the International Tokamak Physics Activity (ITPA) framework will test the approach on other machines with the goal to eventually combine fast-ion measurements on ITER.

Acknowledgements Open access funding provided by Max Planck Society. The authors would like to thank Dr. Kinga Gal for her help in preparation of the manuscript, to Dr. Sergey Bozhenkov, Dr. Dmytro Meshcheriakov, and Dr. Stefan Kragh Nielsen for the fruitful comments and discussion. This work has been carried out within the framework of the EUROfusion Consortium and has received funding from the EURATOM research and training program 2014-2018 under grant agreement No 633053. The views and opinions expressed herein do not necessarily reflect those of the European Commission. We thank the ITPA Energetic Particle Physics Topical Group for its support.

Open Access This article is distributed under the terms of the Creative Commons Attribution 4.0 International License (http://creativecommons.org/licenses/by/4.0/), which permits unrestricted use, distribution, and reproduction in any medium, provided you give appropriate credit to the original author(s) and the source, provide a link to the Creative Commons license, and indicate if changes were made.

\section{References}

V. Afrosimov, Techn. Phys. 5, 1378 (1961)

S. Allison, Rev. Mod. Phys. 30, 1137-1168 (1958). https://doi.org/10.1103/RevModPhys.30.1137

P. Batistoni, Rev. Sci. Instrum. 88, 103505 (2017). https://doi.org/10.1063/1.4991780

R. Behn, Phys. Rev. Lett. 62(24), 2833-2836 (1989). https://doi.org/10.1103/PhysRevLett.62.2833

H.L. Berk, Phys. Rev. Lett. 87, 185002 (2001). https://doi.org/10.1103/PhysRevLett.87.185002

Bindslev, H.: Ph.D. thesis, Ris $\emptyset$ National Laboratory (1992)

H. Bindslev, J. Atmos. Terr. Phys. 58(8), 983-989 (1996). https://doi.org/10.1016/0021-9169(95)00129-8

H. Bindslev, Rev. Sci. Instrum. 70(1), 1093 (1999). https://doi.org/10.1063/1.1149521

H. Bindslev, Phys. Rev. Lett. 83(16), 3206-3209 (1999). https://doi.org/10.1103/PhysRevLett.83.3206

H. Bindslev, Rev. Sci. Instrum. 75(10), 3598 (2004). https://doi.org/10.1063/1.1779620

H. Bindslev, Phys. Rev. Lett. 97(20), 205005 (2006). https://doi.org/10.1103/PhysRevLett.97.205005

H. Bindslev, Plasma Phys. Control. Fusion 49(12B), B551-B562 (2007). https://doi. org/10.1088/0741-3335/49/12B/S52

A. Bortolon, Rev. Sci. Instrum. 81(10), 10 D728 (2010). https://doi.org/10.1063/1.3495768 
H. Brysk, Plasma Phys. 15(7), 611 (1973). https://doi.org/10.1088/0032-1028/15/7/001

E. Carolipio et al., Nucl. Fusion 42, 853 (2002). https://doi.org/10.1088/0029-5515/42/7/308

E.M. Carolipio, Nucl. Fusion 42(7), 853 (2002). https://doi.org/10.1088/0029-5515/42/7/308

E. Carruthers, J. Nucl. Mater. 176-177, 1027-1031 (1990). https://doi.org/10.1016/0022-3115(90)90186 $-\mathrm{Q}$

C. Cazzaniga, Rev. Sci. Instrum. 84, 123505 (2013). https://doi.org/10.1063/1.4847056

C. Cazzaniga, Rev. Sci. Instrum. 85(4), 043506 (2014). https://doi.org/10.1063/1.4870584

C. Cazzaniga, Rev. Sci. Instrum. 85, 11E101 (2014). https://doi.org/10.1063/1.4885356

C. Cazzaniga, Nucl. Instrum. Methods A 778, 20 (2015). https://doi.org/10.1016/j.nima.2015.01.002

M. Cecconello et al., Rev. Sci. Instrum. 81, 10D315 (2010). https://doi.org/10.1063/1.3479038

F.E. Cecil, D.E. Newman, Nucl. Instrum. Methods Phys. Res. 221, 449 (1984). https://doi. org/10.1016/0167-5087(84)90018-8

I. Chugunov, Instrum. Exp. Techn. 51, 166 (2008). https://doi.org/10.1134/S0020441208020024

I.N. Chugunov, Nucl. Fusion 51(8), 083010 (2011). https://doi.org/10.1088/0029-5515/51/8/083010

S. Conroy, Nucl. Fusion 28, 2127 (1988). https://doi.org/10.1088/0029-5515/28/12/001

T. Craciunescu, Nucl. Instrum. Methods A 605, 374 (2009). https://doi.org/10.1016/j.nima.2009.03.224

D. Darrow, Rev. Sci. Instrum. 75(10), 3566-3568 (2004). https://doi.org/10.1063/1.1788876

D. Darrow, Nucl. Fusion 53(1), 013009 (2013). https://doi.org/10.1088/0029-5515/53/1/013009

A.E. Donné, Nucl. Fusion 47(6), S337-S384 (2007). https://doi.org/10.1088/0029-5515/47/6/S07

H.H. Duong, Nucl. Fusion 33(5), 749 (1993). https://doi.org/10.1088/0029-5515/33/5/I06

J. Egedal, H. Bindslev, Phys. Plasmas 11(5), 2191 (2004). https://doi.org/10.1063/1.1697400

V. Erckmann, Fusion Sci. Technol. 52(2), 291-312 (2007). https://doi.org/10.13182/FST07-A1508

J. Eriksson, Nucl. Fusion 55(12), 123026 (2015). https://doi.org/10.1088/0029-5515/55/12/123026

J. Eriksson, Comput. Phys. Commun. 199, 40 (2016). https://doi.org/10.1016/j.cpc.2015.10.010

A. Fasoli, Nucl. Fusion 47(6), S264 (2007). https://doi.org/10.1088/0029-5515/47/6/S05

W.R. Faust, E.G. Harris, Nucl. Fusion 1, $62-63$ (1960). https://doi.org/10.1088/0029-5515/1/1/006

G. Fehrenbacher, Nucl. Instrum. Methods A 372, 239 (1996). https://doi.org/10.1016/01689002(95)01289-3

A. Fernandes, Fusion Eng. Des. 89, 259 (2014). https://doi.org/10.1016/j.fusengdes.2013.08.002

R.K. Fisher, Rev. Sci. Instrum. 81(10), 10D307 (2010). https://doi.org/10.1063/1.3490020

W. Fisher, Phys. Rev. A 28(5), 3121 (1983). https://doi.org/10.1103/PhysRevA.28.3121

R. Fonck, Rev. Sci. Instrum. 56(5), 885-890 (1985). https://doi.org/10.1063/1.1138033

J. Frenje, Plasma Phys. Control. Fusion 40, 1211 (1998). https://doi.org/10.1088/0741-3335/40/7/001

V. Furtula, Rev. Sci. Instrum. 81(10), 10D913 (2010). https://doi.org/10.1063/1.3478881

V. Furtula, Rev. Sci. Instrum. 83(1), 013507 (2012). https://doi.org/10.1063/1.3675886

J. Galdon-Quiroga et al, Plasma Phys. Control. Fusion 60, 105005 (2018). https://doi.org/10.1088/13616587/aad76e

M. García-Muñoz, Nucl. Fusion 47(7), L10-L15 (2007). https://doi.org/10.1088/0029-5515/47/7/L03

M. García-Muñoz, Nucl. Fusion 49(8), 085014 (2009)

M. García-Muñoz, Rev. Sci. Instrum. 80(5), 053503 (2009). https://doi.org/10.1063/1.3121543

M. García-Muñoz, Nucl. Fusion 50, 084004 (2010). https://doi.org/10.1088/0029-5515/50/8/084004

M. García-Muñoz, Phys. Rev. Lett. 104, 185002 (2010). https://doi.org/10.1103/PhysRevLett.104.185002

M. García-Muñoz, Nucl. Fusion 51(10), 103013 (2011)

M. García-Muñoz, Nucl. Fusion 53(12), 123008 (2013). https://doi.org/10.1088/0029-5515/53/12/12300 8

M. García-Muñoz, Plasma Phys. Control. Fusion 55(12), 124014 (2013). https://doi.org/10.1088/0741$3335 / 55 / 12 / 124014$

M. García-Muñoz, Rev. Sci. Instrum. 87, 11D829 (2016). https://doi.org/10.1063/1.4961295

T. Gassner, Phys. Plasmas 19, 032115 (2012). https://doi.org/10.1063/1.3696858

M. Gatu Johnson, Nucl. Instrum. Methods Phys. Res. Sect. A: Accel. Spectrom. Detect. Assoc. Equip. 591(2), 417-430 (2008). https://doi.org/10.1016/j.nima.2008.03.010

B.E. Geiger, Plasma Phys. Control. Fusion 53(6), 065010 (2011). https://doi.org/10.1088/0741$3335 / 53 / 6 / 065010$

B. Geiger, Nucl. Fusion 55, 083001 (2015). https://doi.org/10.1088/0029-5515/55/8/083001

B. Geiger, Plasma Phys. Control. Fusion 57(1), 014018 (2015). https://doi.org/10.1088/07413335/57/1/014018

Geiger, B. et al., Plasma Phys. Control, Fusion (2017)

K. Gentle et al., Rev. Modern Phys. 67(4), 809-836 (1995). https://doi.org/10.1103/RevModPhys.67.809 
S.H. Glenzer et al., Phys. Rev. Lett. 79(7), 1277-1280 (1997). https://doi.org/10.1103/PhysRevLet t.79.1277

N.N. Gorelenkov, Nucl. Fusion 54(12), 125001 (2014). https://doi.org/10.1088/0029-5515/54/12/125001

W.W. Heidbrink et al., Nucl. Fusion 23(7), 917 (1983). https://doi.org/10.1088/0029-5515/23/7/005

W.W. Heidbrink et al., Phys. Rev. Lett 53(20), 1905-1908 (1984). https://doi.org/10.1103/PhysRevLet t.53.1905

W. Heidbrink, G. Sadler, Nucl. Fusion 34(4), 535-615 (1994). https://doi.org/10.1088/0029-5515/34/4/ I07

W.W. Heidbrink, Rev. Sci. Instrum. 81(10), 10D727 (2010). https://doi.org/10.1063/1.3478739

W.W. Heidbrink, Nucl. Fusion 31(9), 1635 (1991). https://doi.org/10.1088/0029-5515/31/9/002

W.W. Heidbrink, Plasma Phys. Control. Fusion 46, 1855-1875 (2004). https://doi. org/10.1088/0741-3335/46/12/005

W.W. Heidbrink, Plasma Phys. Control. Fusion 49, 1457-1475 (2007). https://doi. org/10.1088/0741-3335/49/9/008

W.W. Heidbrink, Commun. Comput. Phys. 10(3), 716-741 (2011). https://doi.org/10.4208/cicp.19081 $0.080211 \mathrm{a}$

C. Hellesen, Plasma Phys. Control. Fusion 52(8), 085013 (2010). https://doi.org/10.1088/0741$3335 / 52 / 8 / 085013$

C. Hellesen, Nucl. Fusion 50(8), 084006 (2010). https://doi.org/10.1088/0029-5515/50/8/084006

C. Hellesen, Nucl. Fusion 53(11), 113009 (2013). https://doi.org/10.1088/0029-5515/53/11/113009

Y.M. Hou, Rev. Sci. Instrum. 87(11), 11E552 (2016). https://doi.org/10.1063/1.4960598

T.P. Hughes, J. Plasma Phys. 42(2), 215-240 (1989). https://doi.org/10.1017/S0022377800014318

M. Ishikawa, Rev. Sci. Instrum. 73(12), 4237 (2002). https://doi.org/10.1063/1.1518145

R. Isler, Phys. Rev. Lett. 38, 1359-1362 (1977). https://doi.org/10.1103/PhysRevLett.38.1359

M. Isobe, Rev. Sci. Instrum. 81, 10D310 (2010). https://doi.org/10.1063/1.3492383

ITER Physics Expert Group, Nucl. Fusion 39(12), 2471-2495 (1999). https://doi. org/10.1088/0029-5515/39/12/305

ITER Physics Expert Group on Diagnostics, Nucl. Fusion 39(12), 2541-2575 (1999). https://doi. org/10.1088/0029-5515/39/12/307

T.E. Ito, Rev. Sci. Instrum. 81(10), 10D327 (2010). https://doi.org/10.1063/1.3502331

A.S. Jacobsen, Rev. Sci. Instrum. 85(11), 11E103 (2014). https://doi.org/10.1063/1.4885477

A.S. Jacobsen, Plasma Phys. Control. Fusion 58(4), 045016 (2016). https://doi.org/10.1088/07413335/58/4/045016

A.S. Jacobsen, Plasma Phys. Control. Fusion 58(4), 042002 (2016). https://doi.org/10.1088/07413335/58/4/042002

A.S. Jacobsen, Rev. Sci. Instrum. 88(7), 073506 (2017). https://doi.org/10.1063/1.4991651

A.S. Jacobsen, Nucl. Fusion 55(5), 053013 (2015). https://doi.org/10.1088/0029-5515/55/5/053013

O. Jarvis, Plasma Phys. Control. Fusion 36, 209 (1994). https://doi.org/10.1088/0741-3335/36/2/002

F. Jaulmes, Nucl. Fusion 56, 112012 (2016). https://doi.org/10.1088/0029-5515/56/11/112012

D. Jimenez-Rey, J. Appl. Phys. 104(6), 064911 (2008). https://doi.org/10.1063/1.2979327

J. Kaellne et al., Phys. Rev. Lett. 85, 1246 (2000). https://doi.org/10.1103/PhysRevLett.85.1246

A.N. Karpushov, Rev. Sci. Instrum. 77(3), 033504 (2006). https://doi.org/10.1063/1.2185151

J. Kim, Rev. Sci. Instrum. 83(10), 10D305 (2012). https://doi.org/10.1063/1.4733550

H. Kimura, Nucl. Fusion 38(9), 1303 (1998). https://doi.org/10.1088/0029-5515/38/9/304

V. Kiptily, Nucl. Fusion 55, 023008 (2015). https://doi.org/10.1088/0029-5515/55/2/023008

V.G. Kiptily, Nucl. Fusion 42, 999-1007 (2002). https://doi.org/10.1088/0029-5515/42/8/308

V.G. Kiptily, Nucl. Fusion 45(5), L21-L25 (2005). https://doi.org/10.1088/0029-5515/45/5/L01

V.G. Kiptily, Plasma Phys. Control. Fusion 48, R59 (2006). https://doi.org/10.1088/0741-3335/48/8/R01

V.G. Kiptily, Nucl. Fusion 50(8), 084001 (2010). https://doi.org/10.1088/0029-5515/50/8/084001

A.I. Kislyakov, Plasma Phys. Control. Fusion 43(12), 1775 (2001). https://doi. org/10.1088/0741-3335/43/12/311

Knoll G.F.: Radiation detection and measurement. Wiley (2010)

Y. Kolesnichenko, Y. Yakovenko, Nucl. Fusion 36(2), 159 (1996). https://doi.org/10.1088/0029-5515/36/2/I04

T. Kondo, Nucl. Fusion 40(9), 1575 (2000). https://doi.org/10.1088/0029-5515/40/9/302

T.E. Kondoh, J. Nucl. Mater. 241-243, 564-568 (1997). https://doi.org/10.1016/S0022-3115(97)80100-6

T. Kondoh, Rev. Sci.Instrum. 74(3), 1642-1645 (2003). https://doi.org/10.1063/1.1532760

T. Kondoh, Fusion Sci. Technol. 51(2T), 62-64 (2007). https://doi.org/10.13182/FST07-A1314

T. Kondoh, Plasma Fusion Res. 2, S1111-S1111 (2007). https://doi.org/10.1585/pfr.2.S1111 
S.B. Korsholm, et al.: In: 2016 41st International Conference on Infrared, Millimeter, and Terahertz waves (IRMMW-THz), pp. 1-2. IEEE (2016). https://doi.org/10.1109/IRMMW-THz.2016.7758537

S.B. Korsholm, Nucl. Instrum. Methods Phys. Res. Sect. A: Accel. Spectrom. Detect. Assoc. Equip. 623(2), 677-680 (2010). https://doi.org/10.1016/j.nima.2010.05.003

S.B. Korsholm, Phys. Rev. Lett. 106(16), 165004 (2011). https://doi.org/10.1103/PhysRevLett.106.16500 4

S. Kubo, Rev. Sci. Instrum. 81(10), 10D535 (2010). https://doi.org/10.1063/1.3481165

Lee, S., Kondoh, T.: In: 6th IAEA Technical Committee meeting on energetic particles in magnetic confinement systems, pp. 134-137. Naka, Japan (2000)

G. Legge, P. Van der Merw, Nucl. Instrum. Methods 63, 157 (1968). https://doi.org/10.1016/0029$554 \mathrm{X}(68) 90321-2$

G. Lehner, F. Pohl, Zeitschrift fur Physik 207, 83 (1967). https://doi.org/10.1007/BF01326097

F. Leipold, Rev. Sci. Instrum. 80(9), 093501 (2009). https://doi.org/10.1063/1.3212567

Leipold, F. et al.: In: 2016 41st International Conference on Infrared, Millimeter, and Terahertz waves (IRMMW-THz) (2016). https://doi.org/10.1109/IRMMW-THz.2016.7758533

D. Liu, Rev. Sci. Instrum. 87(11), 11 D803 (2016). https://doi.org/10.1063/1.4959798

J.S. Machuzak, Rev. Sci. Instrum. 66(1), 484 (1995). https://doi.org/10.1063/1.1146323

D. Manos, Rev. Sci. Instrum. 57(8), 2107-2112 (1986). https://doi.org/10.1063/1.1138756

N.B. Marushchenko, Comput. Phys. Commun. 185(1), 165-176 (2014). https://doi.org/10.1016/j. cpc.2013.09.002

S.S. Medley, Rev. Sci. Instrum. 79(1), 011101 (2008). https://doi.org/10.1063/1.2823259

F. Meo, Rev. Sci. Instrum. 79(10), 10E501 (2008). https://doi.org/10.1063/1.2989140

F. Meo, J. Phys. Conf. Ser. 227, 012010 (2010). https://doi.org/10.1088/1742-6596/227/1/012010

C.A. Michael, Plasma Phys. Control. Fusion 55(9), 095007 (2013). https://doi.org/10.1088/0741$3335 / 55 / 9 / 095007$

S. Michelsen, Rev. Sci. Instrum. 75(10), 3634 (2004). https://doi.org/10.1063/1.1779611

Monte Carlo Code Group: Monte carlo n-particle code. https://mcnp.lanl.gov/

Moseev, D.: Phd thesis, Technical University of Denmark (2011)

D. Moseev, Plasma Phys. Control. Fusion 53(10), 105004 (2011). https://doi.org/10.1088/07413335/53/10/105004

Moseev, D. et al.: In: 38th EPS Conference on Plasma Physics, p. P4.079 (2011)

Muehling, E.: Experimente mit kernspurdetektoren zur diagnostik von protonen und alpha-teilchen aus fusionsplasmen. Diploma thesis, Max-Planck-Institut fuer Plasmaphysik (1982)

Murakami, S. et al.: In: IAEA 2008 Fusion Energy Conference, pp. EX/P8-3 (2008)

T. Murphy, J. Strachan, Nucl. Fusion 25(3), 383 (1985). https://doi.org/10.1088/0029-5515/25/3/012

C.M. Muscatello, Rev. Sci. Instrum. 81(10), 10 D316 (2010). https://doi.org/10.1063/1.3475367

S.K. Nielsen, Phys. Rev. E 77(1), 016407 (2008). https://doi.org/10.1103/PhysRevE.77.016407

S.K. Nielsen, Plasma Phys. Control. Fusion 52(9), 092001 (2010). https://doi.org/10.1088/0741$3335 / 52 / 9 / 092001$

S.K. Nielsen, Nucl. Fusion 51(6), 063014 (2011). https://doi.org/10.1088/0029-5515/51/6/063014

S.K. Nielsen, Plasma Phys. Control. Fusion 57(3), 035009 (2015). https://doi.org/10.1088/07413335/57/3/035009

T. Nishitani, Plasma Phys. Control. Fusion 38, 355 (1996). https://doi.org/10.1088/0741-3335/38/3/010

M. Nishiura, Rev. Sci. Instrum. 75(10), 3646-3648 (2004). https://doi.org/10.1063/1.1779606

M. Nishiura, J. Phys.: Conf. Ser. 227, 012014 (2010). https://doi.org/10.1088/1742-6596/227/1/012014

M. Nishiura, Nucl. Fusion 54(2), 023006 (2014). https://doi.org/10.1088/0029-5515/54/2/023006

M.Nocente, Neutron and gamma ray emission spectroscopy as fast ion diagnostics in fusion plasmas. Ph.D. thesis, Universiti di Milano-Bicocca (2012)

M. Nocente, Nucl. Fusion 50, 055001 (2010). https://doi.org/10.1088/0029-5515/50/5/055001

M. Nocente, Rev. Sci. Instrum. 81(10), 10D321 (2010). https://doi.org/10.1063/1.3501386

M. Nocente, Nucl. Fusion 52(9), 094021 (2012). https://doi.org/10.1088/0029-5515/52/9/094021

M. Nocente, Nucl. Fusion 52(6), 063009 (2012). https://doi.org/10.1088/0029-5515/52/6/063009

M. Nocente, Nucl. Fusion 53, 053010 (2013). https://doi.org/10.1088/0029-5515/53/5/053010

M. Nocente, IEEE Trans. Nucl. Sci. 60(2), 1408-1415 (2013). https://doi.org/10.1109/TNS.2013.22521 89

M. Nocente, Nucl. Fusion 54, 104010 (2014). https://doi.org/10.1088/0029-5515/54/10/104010

M. Nocente, Nucl. Fusion 55(12), 123009 (2015). https://doi.org/10.1088/0029-5515/55/12/123009

M. Nocente, Rev. Sci. Instrum. 86(10), 103501 (2015). https://doi.org/10.1063/1.4931755 
M. Nocente et al., Rev. Sci. Instrum. 87, 11E714 (2016). https://doi.org/10.1063/1.4961073

M. Nocente, Nucl. Fusion 57, 076016 (2017). https://doi.org/10.1088/1741-4326/aa6f7d

K. Ogawa, Rev. Sci. Instrum. 85, 11E110 (2014). https://doi.org/10.1063/1.4890399

F. Orsitto, Rev. Sci. Instrum. 70(1), 1158 (1999). https://doi.org/10.1063/1.1149428

M. Osakabe, Rev. Sci. Instrum. 79(10), 10 E519 (2008). https://doi.org/10.1063/1.2968695

D.C. Pace, Rev. Sci. Instrum. 83(7), 073501 (2012). https://doi.org/10.1063/1.4731655

S.D. Pinches, Phys. Plasmas 22(2), 021807 (2015). https://doi.org/10.1063/1.4908551

M.E. Podestà, Rev. Sci. Instrum. 79(10), 10E521 (2008). https://doi.org/10.1063/1.2956744

I. Proverbio et al., Rev. Sci. Instrum. 81, 10D320 (2010). https://doi.org/10.1063/1.3502037

J. Rasmussen, Plasma Phys. Control. Fusion 57(7), 075014 (2015). https://doi.org/10.1088/07413335/57/7/075014

J. Rasmussen, Nucl. Fusion 56, 112014 (2016). https://doi.org/10.1088/0029-5515/56/11/112014

R.K. Richards, Appl. Phys. Lett. 62(1), 28-30 (1993). https://doi.org/10.1063/1.108808

D. Rigamonti et al., Rev. Sci. Instrum. 87, 11 E717 (2016). https://doi.org/10.1063/1.4961060

M. Rodriguez-Ramos, Plasma Phys. Control. Fusion 59(10), 105009 (2017). https://doi. org/10.1088/1361-6587/aa7e5f

A.L. Roquemore, Rev. Sci. Instrum. 61(10), 3163 (1990). https://doi.org/10.1063/1.1141674

J.S. Ross, Phys. Rev. Lett. 104(10), 105001 (2010). https://doi.org/10.1103/PhysRevLett.104.105001

M. Salewski, O. Asunta, L.-G. Eriksson, H. Bindslev, V. Hynönen, S.B. Korsholm, T. Kurki-Suonio, F. Leipold, F. Meo, P.K. Michelsen, S.K. Nielsen, J. Roenby. Comparison of collective Thomson scattering signals due to fast ions in ITER scenarios with fusion and auxiliary heating. Plasma Phys. Control. Fusion 51(3), 035006 (2009)

M. Salewski, Nucl. Fusion 49(2), 025006 (2009). https://doi.org/10.1088/0029-5515/49/2/025006

M. Salewski, Nucl. Fusion 50(3), 035012 (2010). https://doi.org/10.1088/0029-5515/50/3/035012

M. Salewski, Nucl. Fusion 51(8), 083014 (2011). https://doi.org/10.1088/0029-5515/51/8/083014

M. Salewski, Nucl. Fusion 52(10), 103008 (2012). https://doi.org/10.1088/0029-5515/52/10/103008

M. Salewski, Nucl. Fusion 53(6), 063019 (2013). https://doi.org/10.1088/0029-5515/53/6/063019

M. Salewski, Plasma Phys. Control. Fusion 56(10), 105005 (2014a). https://doi.org/10.1088/07413335/56/10/105005

M. Salewski, Nucl. Fusion 54(2), 023005 (2014b). https://doi.org/10.1088/0029-5515/54/2/023005

M. Salewski, Nucl. Fusion 55(9), 093029 (2015). https://doi.org/10.1088/0029-5515/55/9/093029

M. Salewski, Plasma Phys. Control. Fusion 57(1), 014021 (2015). https://doi.org/10.1088/0741$3335 / 57 / 1 / 014021$

M. Salewski, Nucl. Fusion 56(4), 046009 (2016a). https://doi.org/10.1088/0029-5515/56/4/046009

M. Salewski, Nucl. Fusion 56, 106024 (2016b). https://doi.org/10.1088/0029-5515/56/10/106024

M. Salewski, Nucl. Fusion 57, 056001 (2017). https://doi.org/10.1088/1741-4326/aa60e9

Salewski, M. et al.: Fusion Sci. Technol., pp. 1-14 (2018). https://doi.org/10.1080/15361055.2017.13804 82

E.E. Salpeter, Electron density fluctuations in a plasma. Phys. Rev. 120(3), 1528 (1960). https://doi. org/10.1103/PhysRev.120.1528

M. Sasao, Fusion Sci. Technol. 53(2), 604-639 (2008). https://doi.org/10.13182/FST08-A1681

M. Schneider, Nucl. Fusion 56, 112022 (2016). https://doi.org/10.1088/0029-5515/56/11/112022

P.A. Schneider, Rev. Sci. Instrum. 86(7), 073508 (2015). https://doi.org/10.1063/1.4926886

R. Seki, Plasma Fusion Res. 5, 027 (2010). https://doi.org/10.1585/pfr.5.027

A.G. Shalashov, Plasma Phys. Control. Fusion 45(4), 395-412 (2003). https://doi. org/10.1088/0741-3335/45/4/306

S.E. Sharapov, Phys. Lett. A 289, 127 (2001). https://doi.org/10.1016/S0375-9601(01)00588-6

S.E. Sharapov, Nucl. Fusion 53(10), 104022 (2013). https://doi.org/10.1088/0029-5515/53/10/104022

T. Shimozuma, Fusion Sci. Technol. 58(1), 530-538 (2010). https://doi.org/10.13182/FST58-530

K. Shinohara, Plasma Phys. Control. Fusion 46(7), S31 (2004). https://doi.org/10.1088/0741-3335/46/7/ S03

K. Shinohara, Plasma Fusion Res. 2, 042-042 (2007). https://doi.org/10.1585/pfr.2.042

H. Sjostrand et al., Rev. Sci. Instrum. 77, 10 E717 (2006). https://doi.org/10.1063/1.2336459

J.A. Snipes, Phys. Plasmas 12(5), 056102 (2005). https://doi.org/10.1063/1.1865012

E. Speth, Rep. Progress Phys. 52(1), 57 (1989)

M. Stejner, Rev. Sci. Instrum. 81(10), 10D515 (2010). https://doi.org/10.1063/1.3475540

M. Stejner, Plasma Phys. Control. Fusion 53(6), 065020 (2011). https://doi.org/10.1088/0741$3335 / 53 / 6 / 065020$ 
M. Stejner, Plasma Phys. Control. Fusion 54(1), 015008 (2012). https://doi.org/10.1088/07413335/54/1/015008

M. Stejner, Rev. Sci. Instrum. 83(10), 10E307 (2012). https://doi.org/10.1063/1.4729503

M. Stejner, Nucl. Fusion 52(2), 023011 (2012). https://doi.org/10.1088/0029-5515/52/2/023011

M. Stejner, Plasma Phys. Control. Fusion 55(8), 085002 (2013). https://doi.org/10.1088/07413335/55/8/085002

M. Stejner, Rev. Sci. Instrum. 85(9), 093504 (2014). https://doi.org/10.1063/1.4894199

M. Stejner, Plasma Phys. Control. Fusion 57(6), 062001 (2015). https://doi.org/10.1088/07413335/57/6/062001

M. Stejner et al.: In: 2016 41st International Conference on Infrared, Millimeter, and Terahertz waves (IRMMW-THz) (2016). https://doi.org/10.1109/IRMMW-THz.2016.7758538

J. Strachan et al., Nature 279, 626 (1979). https://doi.org/10.1038/279626a0

E.V. Suvorov, Plasma Phys. Control. Fusion 37(11), 1207-1213 (1995). https://doi. org/10.1088/0741-3335/37/11/002

I. Takafumi, Plasma Fusion Res. 5, S2099-S2099 (2010). https://doi.org/10.1585/pfr.5.S2099

M. Tardocchi et al., Nucl. Fusion 42, 1273 (2002). https://doi.org/10.1088/0029-5515/42/10/311

M. Tardocchi, Phys. Rev. Lett. 107(20), 205002 (2011). https://doi.org/10.1103/PhysRevLett.107.205002

M. Tardocchi, Plasma Phys. Control. Fusion 55(7), 074014 (2013). https://doi.org/10.1088/0741$3335 / 55 / 7 / 074014$

E. Tsakadze, Fusion Sci. Technol. 53(1), 69-76 (2008). https://doi.org/10.13182/FST08-A1654

M.A. Van Zeeland, Nucl. Fusion 46(10), S880 (2006). https://doi.org/10.1088/0029-5515/46/10/S03

M.A. Van Zeeland, Nucl. Fusion 50(8), 084002 (2010). https://doi.org/10.1088/0029-5515/50/8/084002

B. VanDevender et al., IEEE Trans. Nucl. Sci. 61, 2619 (2014). https://doi.org/10.1109/TNS.2014.23570 59

M. Weiland, Plasma Phys. Control. Fusion 58(2), 025012 (2016). https://doi.org/10.1088/0741$3335 / 58 / 2 / 025012$

M. Weiland, Nucl. Fusion 57(11), 116058 (2017). https://doi.org/10.1088/1741-4326/aa7e0a

A. Werner, Rev. Sci. Instrum. 72(1), 780-783 (2001). https://doi.org/10.1063/1.1319357

B. Wolle, Phys. Rep. 312, 1 (1999). https://doi.org/10.1016/S0370-1573(98)00084-2

K.L. Wong et al., Phys. Rev. Lett. 66, 1874-1877 (1991). https://doi.org/10.1103/PhysRevLett.66.1874

P. Woskoboinikow, Int. J. Infrared Millim. Waves 4(2), 205-229 (1983). https://doi.org/10.1007/BF010 08603

G. Wurden, Rev. Sci. Instrum. 66, 901 (1995). https://doi.org/10.1063/1.1146200

Y. Yamaguchi, Nucl. Fusion 55(1), 013002 (2015). https://doi.org/10.1088/0029-5515/55/1/013002

X. Zhang, Nucl. Fusion 54, 104008 (2014). https://doi.org/10.1088/0029-5515/54/10/104008

X. Zhang, Rev. Sci. Instrum. 85, 043503 (2014). https://doi.org/10.1063/1.4869804

G.Q. Zhong, Rev. Sci. Instrum. 87, 11D820 (2016). https://doi.org/10.1063/1.4960814

A. Zimbal et al., Rev. Sci. Instrum. 75, 3553 (2004). https://doi.org/10.1063/1.1787935

S. Zweben, Nucl. Fusion 29(5), 825 (1989). https://doi.org/10.1088/0029-5515/29/5/012

S.J. Zweben, Nucl. Fusion 30(8), 1551 (1990). https://doi.org/10.1088/0029-5515/30/8/013

S.J. Zweben, Nucl. Fusion 35(12), 1445 (1995). https://doi.org/10.1088/0029-5515/35/12/I04

S.J. Zweben, Nucl. Fusion 39(9), 1097 (1999). https://doi.org/10.1088/0029-5515/39/9/304 